\title{
Ion Current Rectification in Nano/Micro-Fluidic Interfaces and Pulsed Glow Discharge Time-of-Flight Mass Spectrometric Chemical Speciation
}

\author{
Han Wang \\ West Virginia University
}

Follow this and additional works at: https://researchrepository.wvu.edu/etd

\section{Recommended Citation}

Wang, Han, "Ion Current Rectification in Nano/Micro-Fluidic Interfaces and Pulsed Glow Discharge Timeof-Flight Mass Spectrometric Chemical Speciation" (2014). Graduate Theses, Dissertations, and Problem Reports. 597.

https://researchrepository.wvu.edu/etd/597

This Dissertation is protected by copyright and/or related rights. It has been brought to you by the The Research Repository @ WVU with permission from the rights-holder(s). You are free to use this Dissertation in any way that is permitted by the copyright and related rights legislation that applies to your use. For other uses you must obtain permission from the rights-holder(s) directly, unless additional rights are indicated by a Creative Commons license in the record and/ or on the work itself. This Dissertation has been accepted for inclusion in WVU Graduate Theses, Dissertations, and Problem Reports collection by an authorized administrator of The Research Repository @ WVU.

For more information, please contact researchrepository@mail.wvu.edu. 


\section{Ion Current Rectification in Nano/Micro-Fluidic Interfaces and Pulsed Glow Discharge Time-of-Flight Mass Spectrometric Chemical Speciation}

Han Wang

Dissertation submitted to the Eberly College of Arts and Sciences at West Virginia University in partial fulfillment of the requirements for the degree of

Doctor of Philosophy in

Chemistry

Approved by

Fred L. King, Ph.D., Chair Patrick S. Callery, Ph.D. Ronald B. Smart, Ph.D. Björn C. G. Söderberg, Ph.D. Stephen J. Valentine, Ph.D.

C. Eugene Bennett Department of Chemistry

Morgantown, West Virginia 2014

Keywords: Nanofluidic/microfluidic interfaces, lon current rectification, Pulsed glow discharge, Mass spectrometry, Chemical speciation

Copyright 2014 Han Wang 


\section{ABSTRACT}

\section{Ion Current Rectification in Nano/Micro-Fluidic Interfaces and Pulsed Glow Discharge Time-of-Flight Mass Spectrometric Chemical Speciation}

\section{Han Wang}

Microfluidics continues to be of interest for analyzing chemical and biological samples because of the disposability, portability, low sample consumption, fast analysis time, and parallel analysis potential for multiple samples in a single device. To improve microfluidic device functionality, integrated systems-nanofluidic/microfluidic interfaces (NMIs) have been fabricated for concentrating samples and performing as molecular gates. Ion current rectification has been confirmed in NMI with an asymmetric system. In chapter 2, the asymmetry of the NMI is systematically altered by varying the inner diameter of the nanocapillary membrane (NCM) reservoir, and the current rectification factor is observed to increase as the inner diameter of NCM reservoir increases. The data provide a new approach to tune the ion current rectification of NMls and strengthen the fundamental knowledge of how these devices function.

Glow discharge mass spectrometry (GDMS) is a well-established technique for the direct analysis of elements in solid samples. The introduction of pulsed glow discharge makes the internal energy of GD plasma tunable so that the specific desired ion signal 
profiles can be obtained and used for chemical speciation. For example, the elemental, structural, and molecular information of organic molecules have been obtained nearly simultaneously using glow discharge time-of-flight mass spectrometry (GDToFMS) coupled with gas chromatography. With careful control of operating parameters of pulsed glow discharge time-of-flight mass spectrometry (GDToFMS), specific cluster ions or ion abundance ratios can be used for speciation of chromium oxides, manganese oxides, and iron oxides.

Chapters 3 and 4 focus on extending the application of pulsed glow discharge timeof-flight mass spectrometry for chemical speciation. Chapter 3 is the analysis of cysteine using pulsed glow discharge time-of-flight mass spectrometry. The characteristic fragment ion at $\mathrm{m} / \mathrm{z} 76$ is used for the quantitative analysis for cysteine. The calibration curve for cysteine standards obtained exhibits good linearity. In chapter 4 , the application of pulsed glow discharge mass spectrometry is extended to direct $\mathrm{Nb}_{x} \mathrm{O} y$ speciation. The effect of variations in temporal and spatial sampling along with variations in operating power on analyte ion signal distributions are studied and discussed. Under optimized conditions, the differentiation of three niobium oxides is achieved by comparison of ion abundance ratios. 


\title{
DEDICATED TO
}

\author{
My parents
}




\section{ACKNOWLEDGEMENTS}

I would first like to thank my advisor, Dr. Fred L. King, for his patience, valuable guidance, and encouragement over the years. With his guidance, I got extensive knowledge and a better understanding of the scientific process. He helped me to become a more confident and independent researcher.

I am grateful to my committee members, Dr. Patrick Callery, Dr. Ronald Smart, Dr. Björn Söderberg, and Dr. Stephen J. Valentine, for their helpful suggestion and valuable discussion.

Also, I want to thank Dr. Aaron Timperman, Dr. Xiaodong Shi and Lisa A. Holland for their help in preparing for my proposal defense and other guidance.

I would like to thank the King group, both present and past members: Xiaqing Xu, Yan Pan, Dr. Teerapat Rojsajjakul, Dr. Guodong Gu, Dr. Yuchen Lu, Dr. Megan DeJesus, Dr. Ting Zhao, Dr. Jennifer Robertson-Honecker for their friendship and help for my research and dissertation. I would also appreciate the help from Dr. Kathleen Reschke, Dr. Brent Reschke, Dr. Xiuli Mao, and Dr. Kyoo Jo for their help on my research and proposal defense. Also, technical support from Allen Burns and Randay Eaglen is gratefully acknowledged too. Without their help, I would not have been able to complete my research projects. I also want to thank Rebecca Secrist, Brenda Prentiss, and Albert Taylor for their hard work and help over the years. 
Foremost, I would like to thank my parents Xuanmin Wang and Xiujun Han for their encouragement, and support over the years. Without their love, I would not be where I am today. 


\section{TABLE OF CONTENTS}

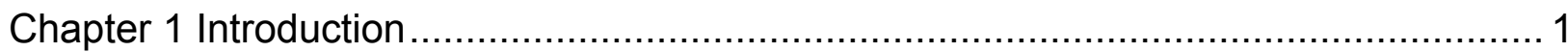

1.1 Ion Current Rectification in Nanofluidic/Microfluidic Interfaces............................ 1

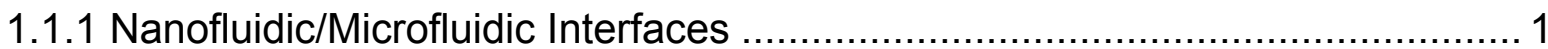

1.1.2 Ion Current Rectification at Nanofluidic/Microfluidic Interfaces ..................... 5

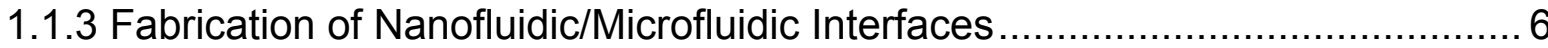

1.2 Chemical Speciation Using Pulsed Glow Discharge Time-of-Flight Mass

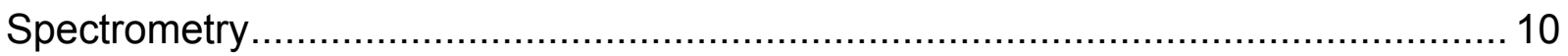

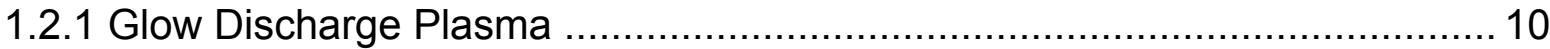

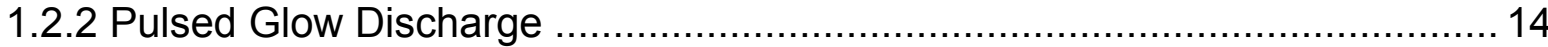

1.2.3 Pulsed Glow Discharge Time of Flight Mass Spectrometry (GD-TOFMS).... 20

1.2.4 Application of Glow Discharge Mass Spectrometry (GDMS) for Chemical

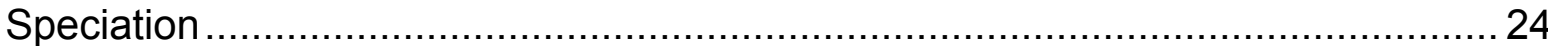

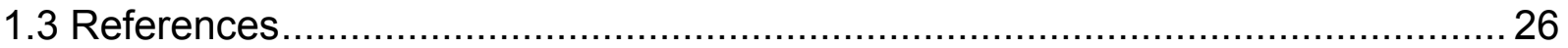

Chapter 2 Controlling the Ion Current Rectification Factor of a Nanofluidic/Microfluidic Interface with Symmetric Nanocapillary Interconnects ............................................. 33

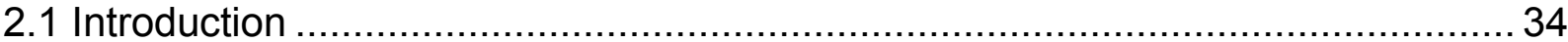

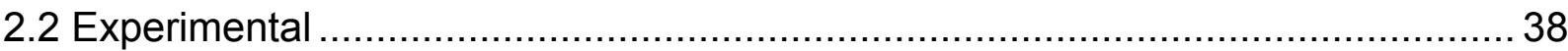

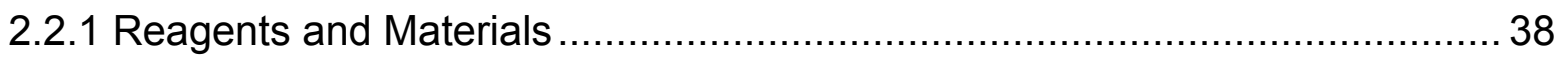


2.2.2 Device Fabrication

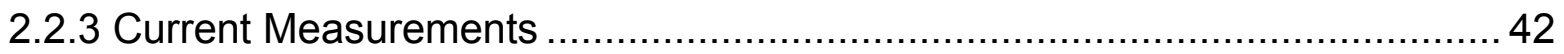

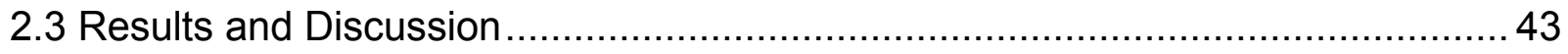

2.3.1 Discussion of I-V plots of Symmetric Devices and Asymmetric Devices ....... 44

2.3.2 Effect of Inner Diameter of NCM Reservoir on Ion Current Rectification Factor 46

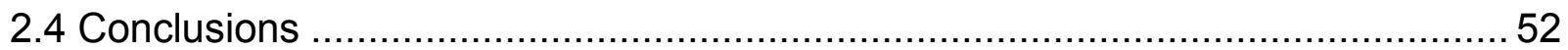

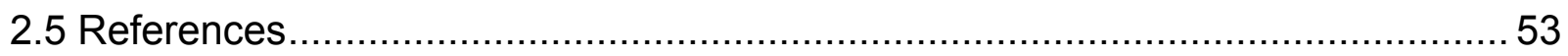

Chapter 3 Analysis of Cysteine Using Pulsed Glow Discharge Time-of-Flight Mass

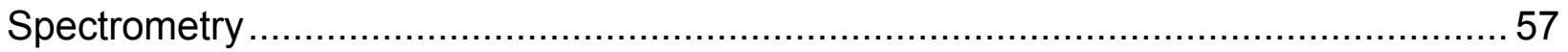

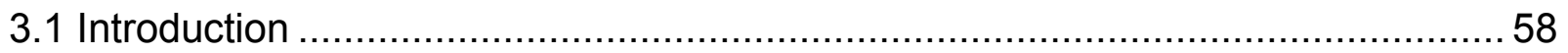

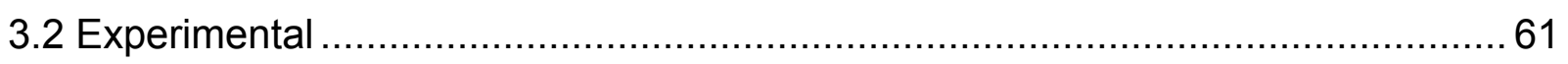

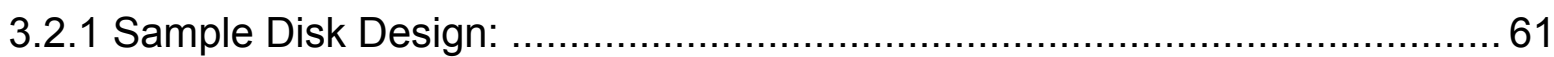

3.2.2 Preparation of Sample Residue on the Sample Disk ................................. 62

3.2.3 Pulsed Glow Discharge TOF Mass Spectrometer ...................................... 63

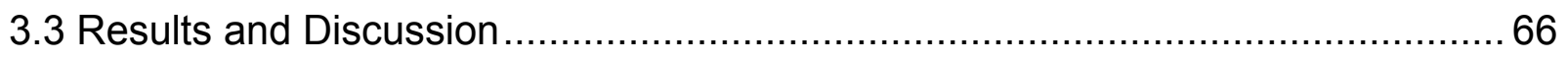

3.3.1 Characteristic Mass Spectra of Cysteine by GD-MS …...............................66

3.3.2 Stability Tests of Characteristic Ion of Cysteine ........................................ 71

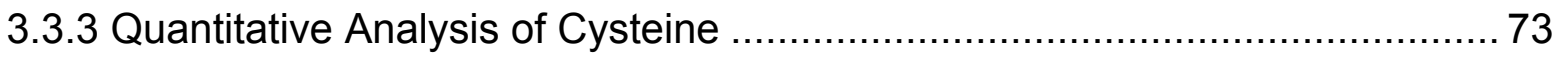

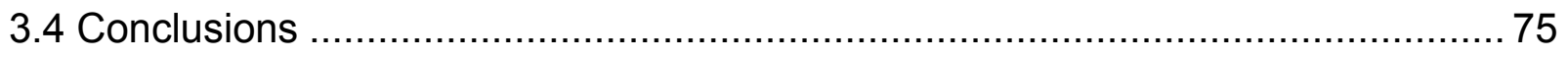


Chapter 4 Direct $\mathrm{Nb}_{x} \mathrm{O}_{\mathrm{y}}$ Speciation in Solid State Materials by Pulsed Glow Discharge

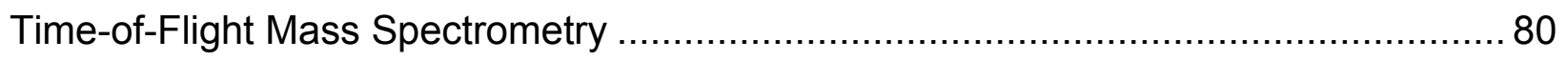

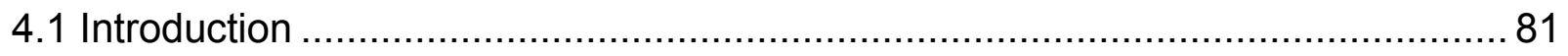

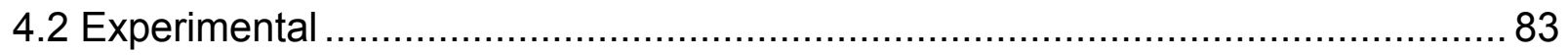

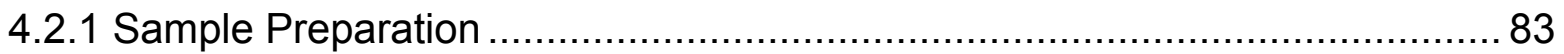

4.2.2 Pulsed Glow Discharge TOF Mass Spectrometer ...................................... 84

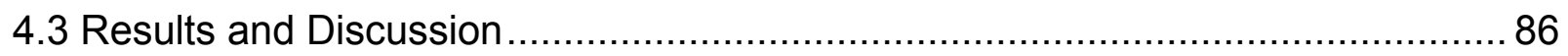

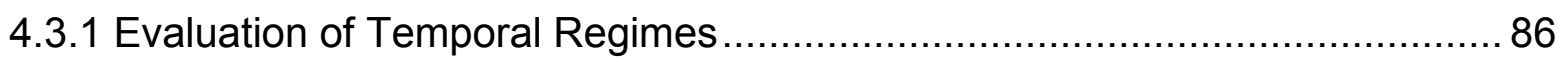

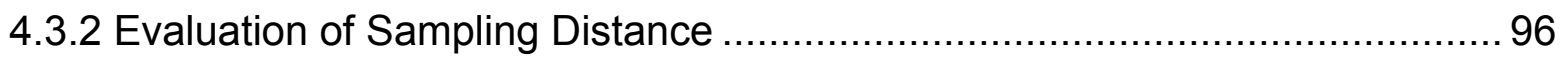

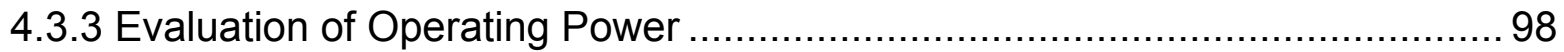

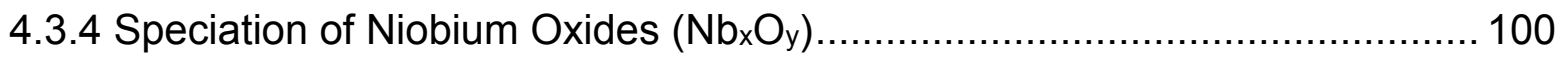

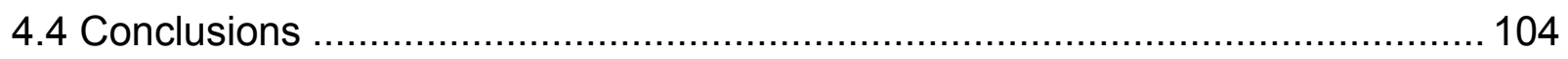

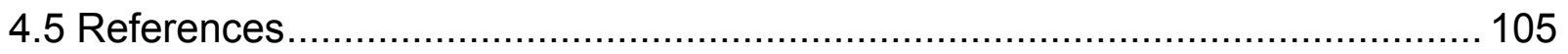




\section{LIST OF FIGURES}

Figure 1.1 Electric double layer created by negatively charged surface and nearby cations. ${ }^{13}$

Figure 1.2 Schematic sketch of the effect of electrolyte concentration on the EDL thickness. 3

Figure 1.3 Schematic of concentration polarization (CP) at a microfluidic/nanofluidic interface. ${ }^{15}$

Figure 1.4 Current-voltage characteristics of an ion current rectifier. 6

Figure 1.5 Procedures for fabrication of photoresist master using photolithography technique. 8

Figure 1.6 Schematic diagram of a glow discharge source. ${ }^{24}$ 11

Figure 1.7 Schematic representation of a millisecond GD power pulse. ${ }^{36}$ 15

Figure 1.8 Temporal ion signal profiles in a pulsed glow discharge: (A) typical applied

voltage, (B) gas species, (C) sputtered species. ${ }^{35}$ 18

Figure 1.9 Diagram of glow discharge time of flight and the setup of data acquisition. ${ }^{44}$ 
Figure 2.1 Schematic diagram of PDMS microfluidic devices. (a) Design of symmetric device. (b) Design of asymmetric device. The ID of NCM reservoir of asymmetric device varies in $150 \mu \mathrm{m}, 300 \mu \mathrm{m}, 625 \mu \mathrm{m}$ and $850 \mu \mathrm{m}$. 40

Figure 2.2 I-V plots for symmetric device and asymmetric devices with different NCM reservoir ID. Each data point is the average quasi-steady currents of 5 runs for each voltage were extracted from the current-time (I-t) plots to build the current voltage plots $(\mathrm{I}-\mathrm{V})$. 45

Figure 2.3 Current rectification factor versus voltage for symmetric and asymmetric devices with different ID of NCM reservoir.

Figure 2.4 Schematic representation of the CP enrich zone and depleted zone at "on" state and "off" state.

Figure 3.1 Mass spectra of (a) Blank and (b) $30 \mu \mathrm{g} / \mathrm{ml}$ cysteine during plateau regime (1.9 ms), and (c) Standard El mass spectrum of cysteine. 68

Figure 3.2 Mass spectra of (a) Blank, (b) $30 \mu \mathrm{g} / \mathrm{ml}$ cysteine during afterpeak (2.1 ms).

Figure 3.3 Temporal intensity profiles of characteristic ion peak $(\mathrm{m} / \mathrm{z} 76)$ of cysteine at different concentrations. 72

Figure 3.4 Calibration curves for cysteine standards based on the peak height intensity of $[\mathrm{M}-\mathrm{COOH}]^{+}(\mathrm{m} / \mathrm{z} 76)$ during afterpeak $(2.1 \mathrm{~ms})$ 74 
Figure 4.1 Time resolved mass spectra of (a) pure copper, (b) $25 \% \mathrm{NbO}+75 \% \mathrm{Cu}$, (c) $25 \% \mathrm{NbO}_{2}+75 \% \mathrm{Cu}$, and (d) $25 \% \mathrm{Nb}_{2} \mathrm{O}_{5}+75 \% \mathrm{Cu}$ samples during the plateau (0.3 ms). Operating conditions: discharge pressure 0.3 Torr, operating power $110 \mathrm{~W}$, pulse duration $5 \mathrm{~ms}$ with $25 \%$ duty cycle, and sampling distance $10 \mathrm{~mm}$ 88

Figure 4.2 Time resolved mass spectra of (a) pure copper, (b) $25 \% \mathrm{NbO}+75 \% \mathrm{Cu}$, (c) $25 \% \mathrm{NbO}_{2}+75 \% \mathrm{Cu}$, and (d) $25 \% \mathrm{Nb}_{2} \mathrm{O}_{5}+75 \% \mathrm{Cu}$ samples during the plateau (4.9 ms). Operating conditions: discharge pressure 0.3 Torr, operating power $110 \mathrm{~W}$, pulse duration $5 \mathrm{~ms}$ with $25 \%$ duty cycle, and sampling distance $10 \mathrm{~mm}$.

Figure 4.3 Time resolved mass spectra of (a) pure copper, (b) $25 \% \mathrm{NbO}+75 \% \mathrm{Cu}$, (c) $25 \% \mathrm{NbO}_{2}+75 \% \mathrm{Cu}$, and (d) $25 \% \mathrm{Nb}_{2} \mathrm{O}_{5}+75 \% \mathrm{Cu}$ samples during the plateau (5.2 ms). Operating conditions: discharge pressure 0.3 Torr, operating power $110 \mathrm{~W}$, pulse duration $5 \mathrm{~ms}$ with $25 \%$ duty cycle, and sampling distance $10 \mathrm{~mm}$ 94

Figure 4.4 Effect of delay time on $\mathrm{Nb}^{+}, \mathrm{NbO}^{+}$and $\mathrm{NbO}^{2+}$ in afterpeak regime using $25 \% \mathrm{NbO}+75 \% \mathrm{Cu}$ pellet. Operating conditions: discharge pressure 0.3 Torr, operating power $110 \mathrm{~W}$, pulse duration $5 \mathrm{~ms}$ with $25 \%$ duty cycle, and sampling distance $10 \mathrm{~mm}$. Each data point is the average intensities of 3 measurements for each time. 96

Figure 4.5 Effect of sampling distance on the ion signal profiles for representative $25 \% \mathrm{NbO}+75 \% \mathrm{Cu}$ pellet in afterpeak regime (5.2 ms). Operating conditions: discharge pressure 0.3 Torr, operating power $110 \mathrm{~W}$ and pulse duration $5 \mathrm{~ms}$ with $25 \%$ duty cycle. Each data point is the average intensities of 3 measurements for each distance. 98 
Figure 4.6 Effect of operating power on $\mathrm{Nb}^{+}, \mathrm{NbO}^{+}$and $\mathrm{NbO}^{2+}$ in afterpeak regime $(5.2$ ms) using $25 \% \mathrm{NbO}+75 \% \mathrm{Cu}$ pellet. Operating conditions: discharge pressure 0.3 Torr, pulse duration $5 \mathrm{~ms}$ with $25 \%$ duty cycle, and sampling distance $10 \mathrm{~mm}$. Each data point is the average intensities of 3 measurements for each operating power.

Figure 4.7 $\mathrm{NbO}_{x}^{+}(x=1,2)$ and $\mathrm{Nb}^{+}$peak intensity ratios for $\mathrm{NbO}, \mathrm{NbO}_{2}$, and $\mathrm{Nb}_{2} \mathrm{O}_{5}$ during the afterpeak (5.2 ms). Operating conditions: discharge pressure 0.3 Torr, operating power $110 \mathrm{~W}$, pulse duration $5 \mathrm{~ms}$ with $25 \%$ duty cycle, and sampling distance $10 \mathrm{~mm}$. Each data point is the average of 6 measurements for each species 


\section{LIST OF TABLES}

Table 2.1 Cross-sectional area of NCM reservoir and current rectification factor at \pm 150

$\mathrm{V}$ for asymmetric devices with different ID of NCM reservoir. 46

Table 3.1 TOF-MS operating parameters 65

Table 4.1 Pulsed RF GD TOF-MS operating parameters 85 


\section{Chapter 1}

\section{Introduction}

\subsection{Ion Current Rectification in Nanofluidic/Microfluidic Interfaces}

\subsubsection{Nanofluidic/Microfluidic Interfaces}

Microfluidics continues to be of great interest for the analysis of chemical and biological samples because of the disposability, portability, low sample consumption, fast analysis time, and capability of parallel analysis for multiple samples in a single device. Various processes have been successfully performed in the microfluidic systems, such as separation, purification and chemical reaction. ${ }^{1-3}$ To improve microfluidic device functionality, nanofluidic elements can be integrated into the microfluidic devices to make nanofluidic/microfluidic interfaces (NMIs). One of the important applications of NMls is as a concentrator for sample enrichment. ${ }^{2-6}$ In addition, NMls are proven to perform as molecular gates..$^{7-9}$ These functions of NMls originate from their unique mass transport properties induced by the ion permselectivity of nanofluidic elements. ${ }^{4-5,10}$

The ion permselectivity of nanofluidic elements is related to their size and thickness of electric double layer. ${ }^{11}$ The electric double layer is the region in the electrolyte solution close to the charged surface of channel that has an excess of 
counterions (ions of opposite charge to that of the surface), compared with coions, to balance the surface charge. The double layer at a negatively charged surface is shown in Figure 1.1. The electric double layer consists of the Stern layer and the diffuse layer. The Stern layer, tightly absorbed by charged surface, is an immobile layer of cations. The remaining negative charge is balanced by large number of cations exist in diffuse layer which is free to move. ${ }^{12-13}$

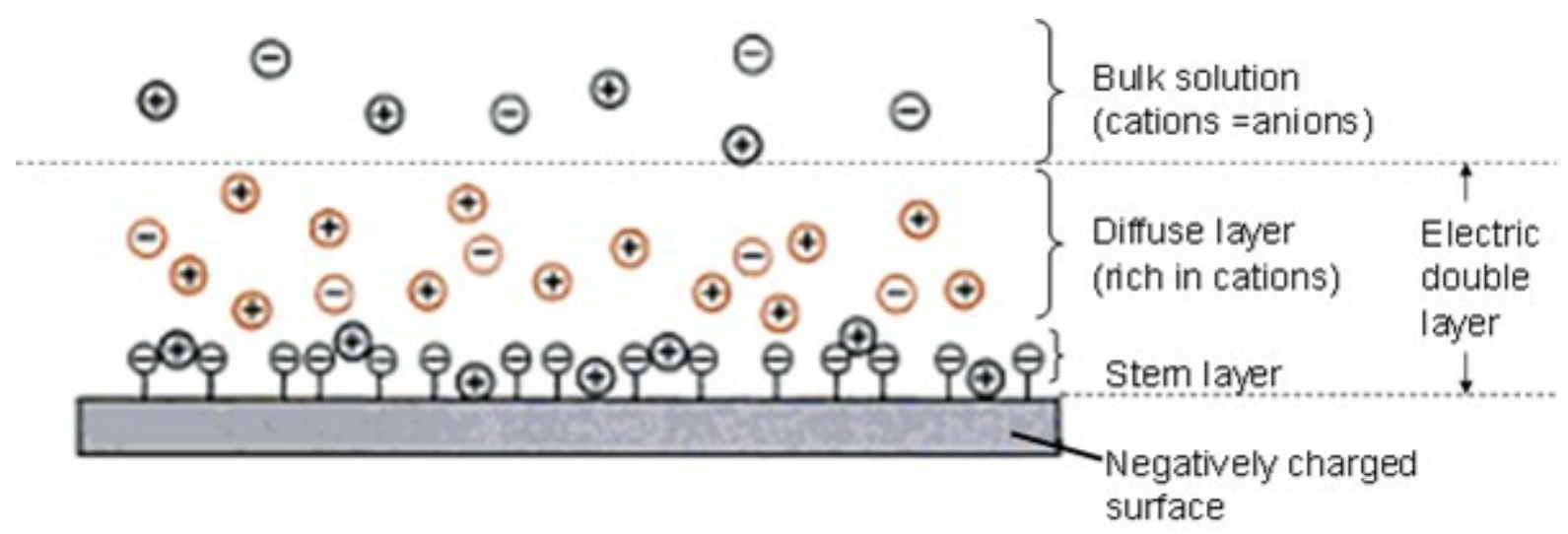

Figure 1.1 Electric double layer created by negatively charged surface and nearby cations. ${ }^{13}$

Ion transport through nanochannels can be much different than in larger systems because the double layer thickness can be on the same order of magnitude as the nanochannel radius. The thickness of the electric double layer is inversely proportional to the square root of the electrolyte concentration. ${ }^{10-12}$ As shown in Figure 1.2, the electric double layer gets thicker with lower concentration of solution. When the double layer thickness exceeds the nanochannel radius, double layer overlap is said to exist. With the 
appropriate nanochannel diameter and ionic concentration, the surface charge of the nanochannel cannot be totally compensated by solution. Consequently, the charged surface of nanochannel repels the coions, exhibits preferential transport of counterions and becomes permselective..$^{10-11,14}$

\section{microchannel nanochannel microchannel microchannel nanochannel microchannel}

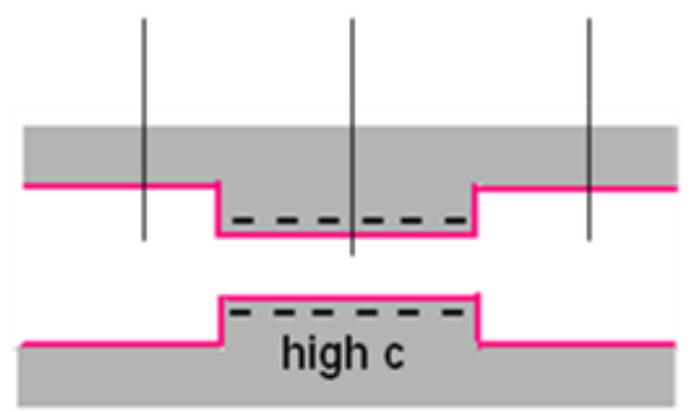

c - concentration of electrolyte

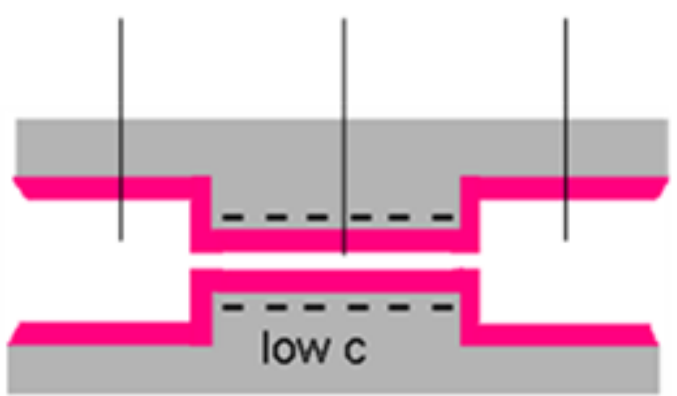

- Electric double layer (EDL) close to negatively charged surface (rich in cations)

Figure 1.2 Schematic sketch of the effect of electrolyte concentration on the EDL thickness.

When the ion permeselctive nanofluidic elements are incorporated into microfluidic devices to form NMls, concentration polarization (CP) develops upon the application of an electric field. A permselective material can be either cation permselective or anion permselective. A cation permselective material readily passes cations, and hinders transport of anions; while an anion permselective material readily passes anions, and hinders transport of cations. ${ }^{10-11}$ As shown in Figure 1.3, a nanocapillary with excess negative surface charge is cation permselective. When the electric field is applied, it will 
allow the cations (counterions) to migrate through the nanocapillary more readily than anions (coions). On the cathodic side of nanocapillary, the transport of anions toward the interface is faster than that into the nanocapillary. Consequently, the anions are accumulated at the cathodic side of nanocapillary and cations are also enriched at the cathodic side to maintain the electroneutrality. On the opposite side of the nanocapillary, the cations move into the nanocapillary and are not replaced by cations moving toward the interface as rapidly as they move into the nanocapillary. This flux imbalance creates a zone that is depleted in cations and anions, to maintain charge neutrality. Therefore, $\mathrm{CP}$ induces an ion depleted zone on the anodic side of the nanocapillary and an ion enriched zone on the cathodic side of the nanocapillary. ${ }^{10-15} \mathrm{CP}$ at NMls can be used for ion current rectification. ${ }^{16-17}$

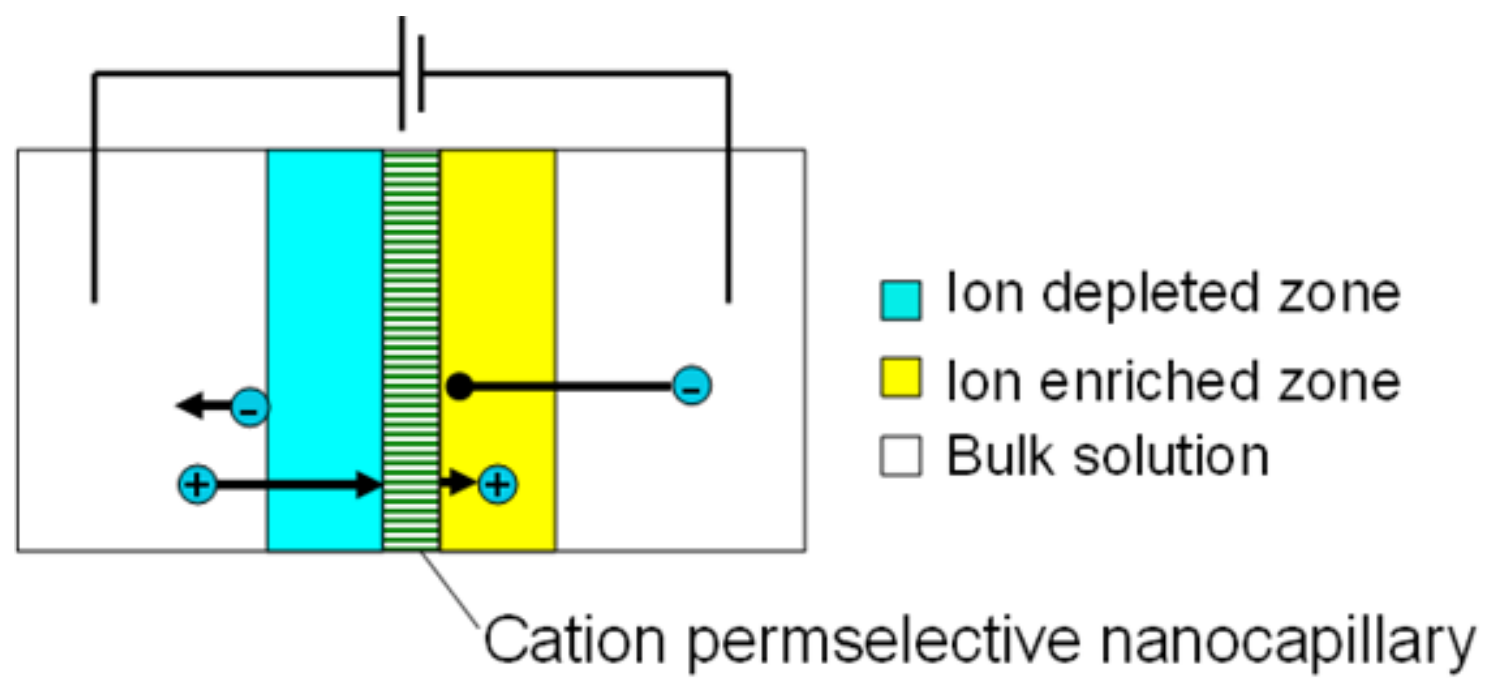

Figure 1.3 Schematic of concentration polarization (CP) at a microfluidic/nanofluidic interface. $^{15}$ 


\subsubsection{Ion Current Rectification at Nanofluidic/Microfluidic Interfaces}

Ion current rectification produces a greater flow of current in one direction than in the opposite direction. It is characterized by a diode-like asymmetric current-voltage (I-V) curve, as illustrated in Figure 1.4. The degree of ion current rectification is evaluated by a current rectification factor, which is the ratio of "on" state current to "off "state current at voltages with opposite polarities but same magnitude. Ion current rectification has been achieved by incorporating asymmetry into the system, which is most commonly achieved through differential coatings or the use of asymmetric channel geometry. ${ }^{14,}$ 18-20 Additionally, ion current rectification has been observed in systems that are not strictly nanofluidic, including conical microscale pores, and nanofluidic/microfluidic interfaces (NMIs). ${ }^{16-17,21}$ 


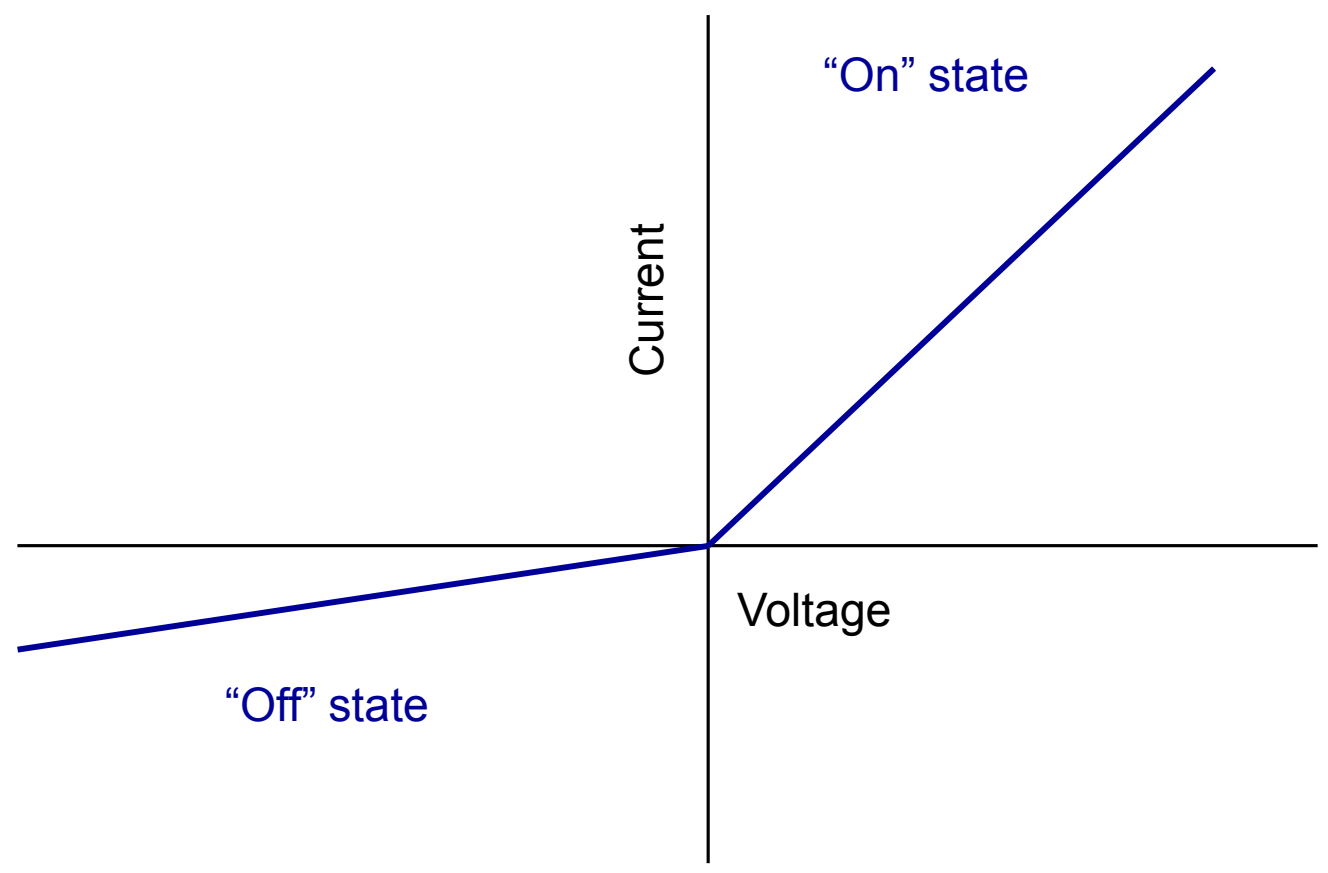

Figure 1.4 Current-voltage characteristics of an ion current rectifier.

\subsubsection{Fabrication of Nanofluidic/Microfluidic Interfaces}

Microfluidic devices are usually made from common materials, like glass, polydimethylsiloxane (PDMS), and polydimethylmethacrylate (PMMA). Fabrication of glass devices is achieved usually through photolithography and wet chemical etching techniques. ${ }^{15}$ The devices used in present work are PDMS devices, and the fabrication procedures are described below. 
Generally, PDMS devices with microchannels are fabricated through three main procedures: (1) fabrication of a master, (2) fabrication of PDMS pieces, and (3) irreversible bonding between PDMS pieces. First, a silicon wafer is used to make the master which has the micro-sized pattern to be used to make a microchannel in the second step. The fabrication of the silicon master is achieved using photolithography technique, which uses ultraviolet (UV) light to transfer the pattern from a photomask to the silicon wafer, as shown in Figure1.5. The transparent areas of the photomask are used to define the microfluidic channels. At first, the photoresist is spin coated to the desired thickness, and pre-baked on the hot plate. After that, the photoresist coated silicon wafer is covered by the photomask, polymerized by exposure to UV-light for a set amount of time, and post-baked. Finally, the unexposed part is removed by immersing the wafer in developer. In this way, the photoresist master is formed with desired patterns on it. Next, the PDMS pieces are made separately and then assembled to fabricate the desired device. A mixture of PDMS prepolymer and the curing agent is degassed to remove all the bubbles and poured to suitable master and mold to make PDMS pieces. To make the thinner patterned layer of the PDMS, the PDMS mixture on the photoresist master usually is spin coated and cured. Finally, the PDMS pieces are plasma treated using a surface Plasma Cleaner and irreversibly bonded together. 


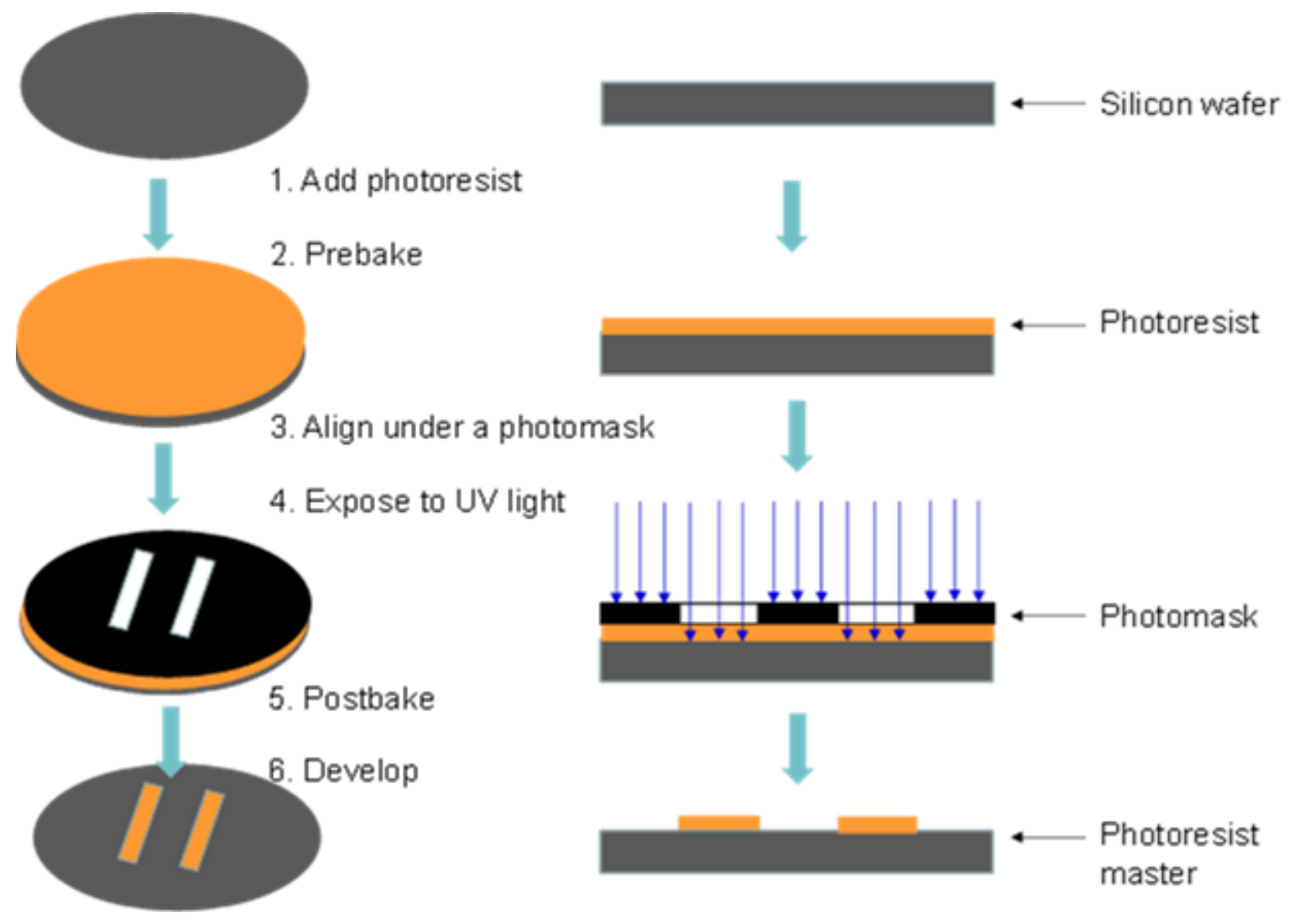

Figure 1.5 Procedures for fabrication of photoresist master using photolithography technique.

Nanofluidic elements used in the NMls are usually nanochannels or nanocapillary membranes (NCMs). Generally, the nanochannel is fabricated directly from the substrate using machining or etching technique. In addition, electric breakdown is used to produce 
the nanochannel. The nanofluidic element used in chapter 2 is a track-etched polycarbonate NCM membrane, which can be purchased with a range of different pore sizes from $10 \mathrm{~nm}$ to $20 \mu \mathrm{m}$. The $10 \mathrm{~nm}$ pores of NCM are usually smaller than most nanochannels formed in the microfluidic devices. ${ }^{4,15}$ 


\subsection{Chemical Speciation Using Pulsed Glow Discharge Time-of-Flight Mass Spectrometry}

\subsubsection{Glow Discharge Plasma}

The basic components and structure of a glow discharge source are shown in Figure 1.6. Generally, the analytical glow discharge source is filled with inert gas (usually Ar) at a pressure about 0.1-10.0 Torr. The sample serves as cathode, and the grounded glow discharge chamber wall is designated as anode in most glow discharge sources. A potential difference $(250-2000 \mathrm{~V})$ is applied between cathode $(-)$ and anode $(+)$ to initiate electrical breakdown of the discharge gas and form the luminous discharge, known as glow discharge plasma. This electrical breakdown causes formation of free electrons and positively charged ions (e.g. $\left.\mathrm{Ar}^{+}\right) \cdot{ }^{22-23}$ 


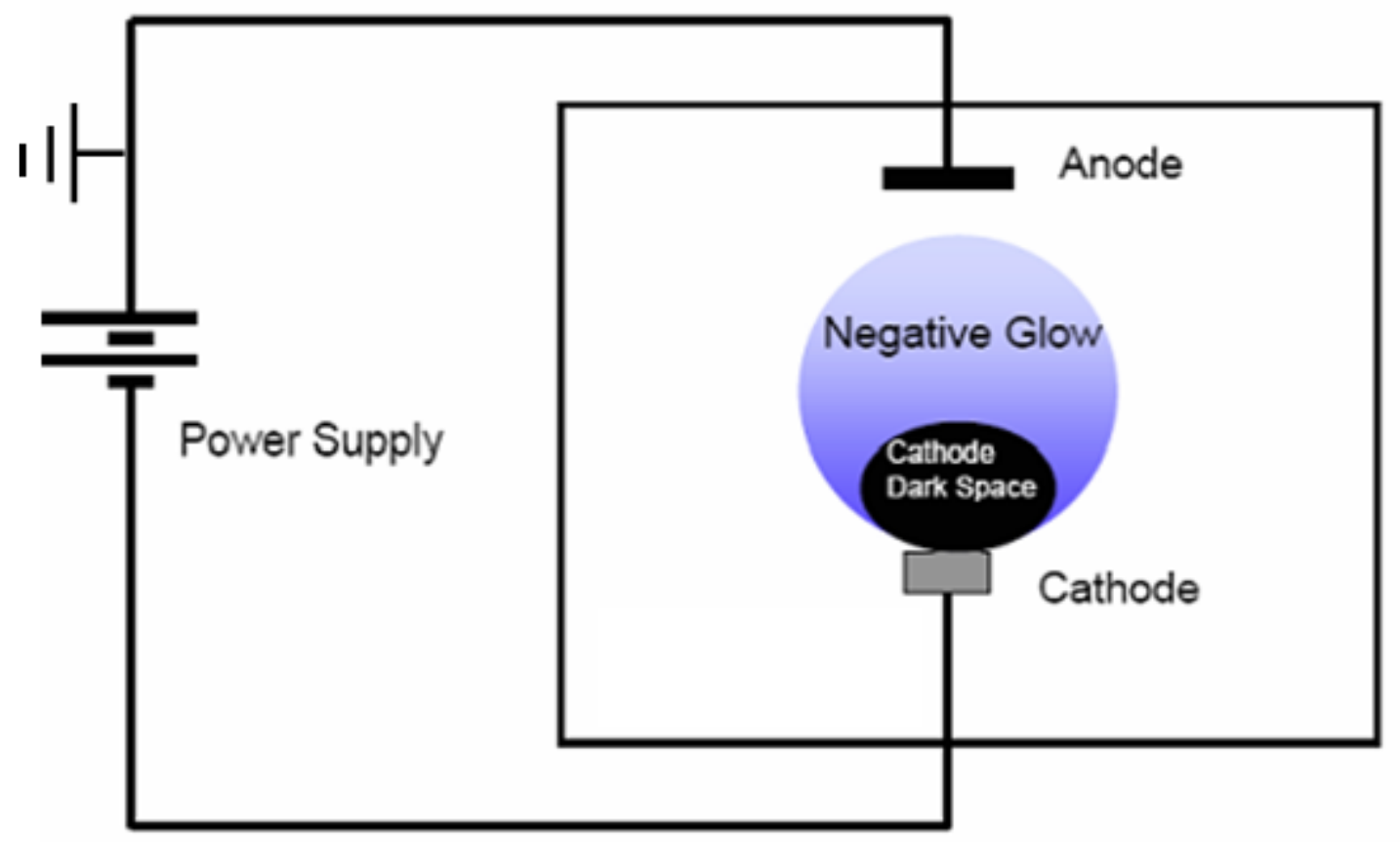

Figure 1.6 Schematic diagram of a glow discharge source. ${ }^{24}$

The two plasma regions shown in Figure 1.6 are most relevant in analytical chemistry: the cathode dark space and the negative glow. In addition to those regions, glow discharge plasmas may also exhibit additional regions - Aston dark space, cathode layer, faraday dark space, positive column, anode dark space and anode glow. However, these regions provide little useful analytical information. ${ }^{22}$

The cathode dark space is a thin layer with low luminosity near the cathode. This region is dark due to the low possibility for collisional excitation required for emission of light. ${ }^{25}$ The majority $(80 \%)$ of the voltage is dropped across this dark region because electrons are repelled from cathode to form a positive space charge surrounding cathode. 
In this strong electric field, argon ions are accelerated toward the negatively charged cathode, strike the cathode surface, and transfer their kinetic energy to the surface. The solid sample is converted into the gas phase by cathodic sputtering. When near-surface analyte species obtain enough energy to overcome lattice binding, cathodic sputtering occurs, releasing neutral species of cathode surface material, clusters and secondary electrons. ${ }^{26-28}$ The sputtered neutral cathode species can diffuse into negative glow region. The secondary electrons ejected in cathodic sputtering are accelerated to the negative glow, contributing both electrons and energy to the discharge. ${ }^{29}$

As shown in Figure 1.6, adjacent to the cathode dark space is the bright blue/purple (if using argon as discharge gas) luminous region known as negative glow, which is essentially field free. Generally, this region contains various species: fast (energetic) secondary electrons, slow (thermalized) electrons, metastable atoms, and ions. Therefore, many collisional excitation and ionization reactions occur in the negative glow region, which makes glow discharge very useful in analytical chemistry applications. For example, emission of characteristic glow from atoms excited by electrons can be detected by atomic emission spectroscopy. ${ }^{22,25}$ There are three common collisional processes in glow discharge that provide ionization: electron ionization, Penning ionization and charge transfer ionization. The majority of sample ions are generated through electron ionization and Penning ionization. These ionization processes make the glow discharge useful for mass spectrometric analysis. ${ }^{23}$

Electron ionization occurs for discharge gas, argon, and sample species. Fast electrons were responsible for electron ionization in glow discharge plasma. Fast electrons obtain high kinetic energy through acceleration across the cathode dark space. 
The fast primary electron collides inelastically with a neutral species, $\mathrm{M}^{0}$, to remove one electron from the neutral, producing a positively charged ion and two slow electrons Equation (1-1). One slow electron is emitted from ionization reaction, and the other slow electron is produced from primary electron which has lost energy through inelastic collision with neutral particle. ${ }^{22}$ If the energy of fast electron is higher than ionization potential of colliding neutral particle, and the particle is polyatomic, fragmentation can occur in addition to ionization and excitation. ${ }^{23}$, 30 Fast electrons are important for sustaining glow discharge plasma because they can collide with argon atoms in discharge to generate more argon ions and new electrons through electron ionization.

$$
M^{0}+e^{-}(\text {fast }) \rightarrow M^{+}+2 e^{-}(\text {slow })
$$

Slow (thermalized) electrons are important for ionization process because they are necessary to generate metastable argon atoms, $A r^{m}$, which are the Penning ionization reagent. Metastable argon atoms are formed by recombination of the argon ions with slow electrons Equation (1-2). In turn, the sputtered neutrals can collide with metastable argon atoms to produce the charged ions, a process known as Penning ionization Equation (1-3). This process only occurs when the energy of metastable argon is larger than the ionization potential of the analyte..$^{22}$ The energy of metastable argon is 11.55 and $11.72 \mathrm{eV}$, which can ionize nearly all elements. However, atmospheric impurities (i.e. $\mathrm{H}_{2} \mathrm{O}$ and $\mathrm{CO}_{2}$ ) in the discharge gas cannot be ionized by metastable argon. This is one advantage to use argon as glow discharge gas. ${ }^{27,} 31$ Additionally, Penning ionization contributes $40-80 \%$ of sputtered species ionization in a continuous glow discharge operating at $0.4-1.2$ Torr, $1-5 \mathrm{~mA}$, and 500-3500 V. ${ }^{32}$ 


$$
\begin{gathered}
\left.\mathrm{Ar}^{+}+\mathrm{e}^{-} \text {(slow }\right) \rightarrow \mathrm{Ar}^{\mathrm{m}} \\
\mathrm{M}^{0}+\mathrm{Ar}^{\mathrm{m}} \rightarrow \mathrm{M}^{+}+\mathrm{Ar} \mathrm{r}^{0}+\mathrm{e}^{-}
\end{gathered}
$$

In addition, Charge transfer (exchange) can be another mechanism for ionization in glow discharge. The sample can be ionized by transferring one electron to argon ion when a neutral collides with an argon ion. This process is known as charge transfer Equation (1-4). Unlike electron ionization and Penning ionization, charge transfer is a selective ionization mechanism because it only occurs when there is a small difference of energy between argon ion $(15.8 \mathrm{eV}){ }^{22}$ and product ion. Therefore, ionizations by electron ionization and Penning ionization are more favorable compared to charge transfer. ${ }^{23}$

$$
\mathrm{M}+\mathrm{Ar}^{+} \rightarrow \mathrm{M}^{+}+\mathrm{Ar}
$$

\subsubsection{Pulsed Glow Discharge}

In glow discharge, a higher ion signal and lower limit of detection (LOD) can be achieved by increasing the operating power. This is because it results in an increase in the sputtering rate and ionization efficiency. ${ }^{33}$ For a glow discharge operated with continuous power, the maximum power is limited by overheating of the cathode, thermal emission, sample cracking, and instable glow discharge plasma. ${ }^{22}$ To solve this problem, a pulsed glow discharge is used to produce higher instantaneous power. Instead of continuous cathodic sputtering, the power is switched between "on" and "off" like a square-wave shape Figure 1.7, known as pulsed glow discharge. Figure 1.7 shows a 
pulse period with $50 \%$ duty cycle. The cathodic temperature increasing during the "on" period is followed by cathodic cooling during the "off" period to avoid overheating. Therefore, the pulsed glow discharge can operate at higher instantaneous powers. In turn, more analytical species are sputtered and ionized in the glow discharge, enhancing the analytical signal and improving LOD and sensitivity. Compared to continuous glow discharge, the ion signal intensity was increased about 2 orders of magnitude by pulsed glow discharge with $50 \%$ duty cycle. Modulation of duty cycle allows adjustment of ion signal. There are more benefits for pulsed glow discharge plasma to be obtained by understanding three distinct temporal regimes: the prepeak, plateau, and afterpeak. ${ }^{34-35}$

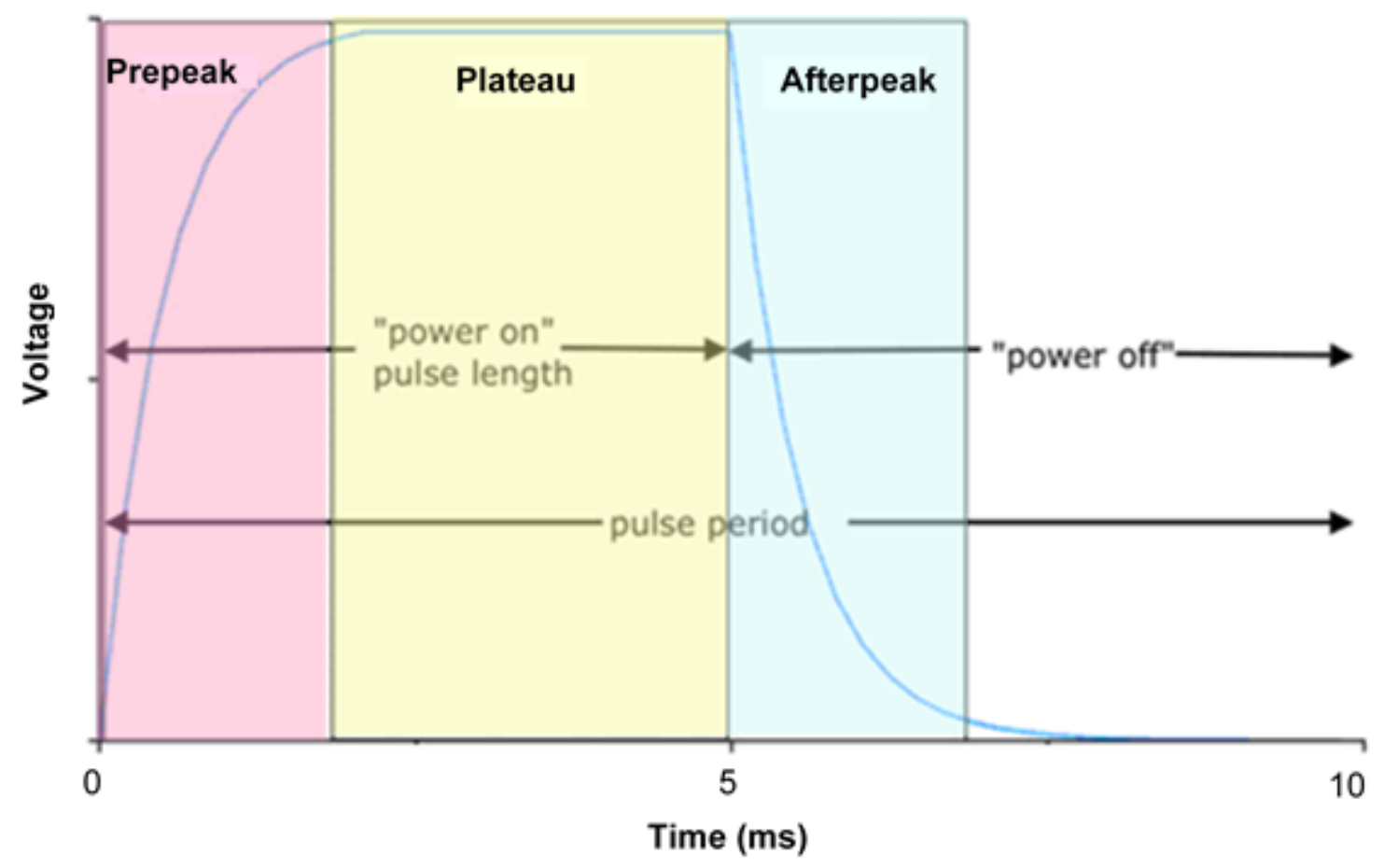

Figure 1.7 Schematic representation of a millisecond GD power pulse. ${ }^{36}$ 


\subsubsection{Prepeak Regime}

As described previously, the electrical breakdown of discharge gas occurs when the power is applied. The resulting energetic electrons can ionize gas species, which already exist in the glow discharge chamber. This process induces the surge in ion intensity of gas species (e.g. discharge gas Ar and contaminant gases $\mathrm{H}_{2} \mathrm{O}, \mathrm{CO}_{2}$, and $\mathrm{N}_{2}$ ), termed as the prepeak regime (Figure $1.8 \mathrm{~B}$ ). ${ }^{34,37}$ This process is dominated by electron ionization. The signals of sputtered species appear after the gas species because it takes some time for sputtered species to diffuse into negative glow and to be ionized. ${ }^{37}$

\subsubsection{Plateau regime}

After the gas species ion signals reach maxima, they start to decline to a relatively stable value. Meanwhile, the sputtered species signals show up, increase, and reach a steady state. Ion signals of both gas species and sputtered species exist and remain at the steady state value until termination of the applied voltage. This period is called plateau regime. In this temporal region, the energetic electrons, metastable argon atoms, and argon ions all exist in a quasi-equilibrium state. Therefore, ionization in the plateau regime of pulsed glow discharge is a mixture of electron ionization, Penning ionization and charge transfer ionization, which is similar to a continuous glow discharge. ${ }^{29,37}$ 
Klingler et al. reported that mass spectrum from plateau regime is analogous to that from continuous glow discharge taken at the same voltage. ${ }^{34}$

\subsubsection{Afterpeak regime}

After the applied voltage terminates, the sudden increase of ion signals for sputtered species indicates the beginning of the afterpeak regime of the pulsed glow discharge. Once ion signals of sputtered species reach the peak value, they decrease to the baseline of mass spectra. Instead of formation of a peak, the ion signals of gas species decay directly to the baseline value after termination of the power. ${ }^{35}$ The variation of ion signals for sputtered species and gas species in afterpeak portion are due to the change of the ionization environment in glow discharge plasma. Following the power off, the energetic electrons cannot be produced any more, and their population declines rapidly by inelastic collisions and diffusion to the wall, which makes the electron ionization extinguish. The thermalized electrons from collisions recombine with the argon ion to generate metastable argon atoms, which can engage in Penning ionization. The charge transfer halts because of the loss of argon ions. Due to a large amount of metastable argon atoms, Penning ionization becomes the dominant ionization mechanism in afterpeak regime. ${ }^{38-39}$ As mentioned before, metastable argon atoms can only ionize the species whose ionization potentials are lower than energy of metastable argon atoms (11.55 and $11.72 \mathrm{eV}$. Consequently, the ionization potentials of contaminant gas species (i.e. $\mathrm{H}_{2} \mathrm{O} 12.6 \mathrm{eV}, \mathrm{CO}_{2} 13.8 \mathrm{eV}$, and $\mathrm{N}_{2} 15.6 \mathrm{eV}$ ) and discharge gas argon (15.8 eV) are 
too high to be ionized by argon metastable argon atoms, so their ion signals decay fast after termination of the power. ${ }^{31}$ Therefore, analytically cleaner mass spectrum can be obtained in afterpeak regime. For the analytes which can be ionized by Penning ionization, their signals rise because of the increased population of metastable argon atoms. Moreover, the reduced electron density also contributes to the enhanced analyte ion signals by reducing the loss of analyte ions induced by recombination with electrons. ${ }^{36}$

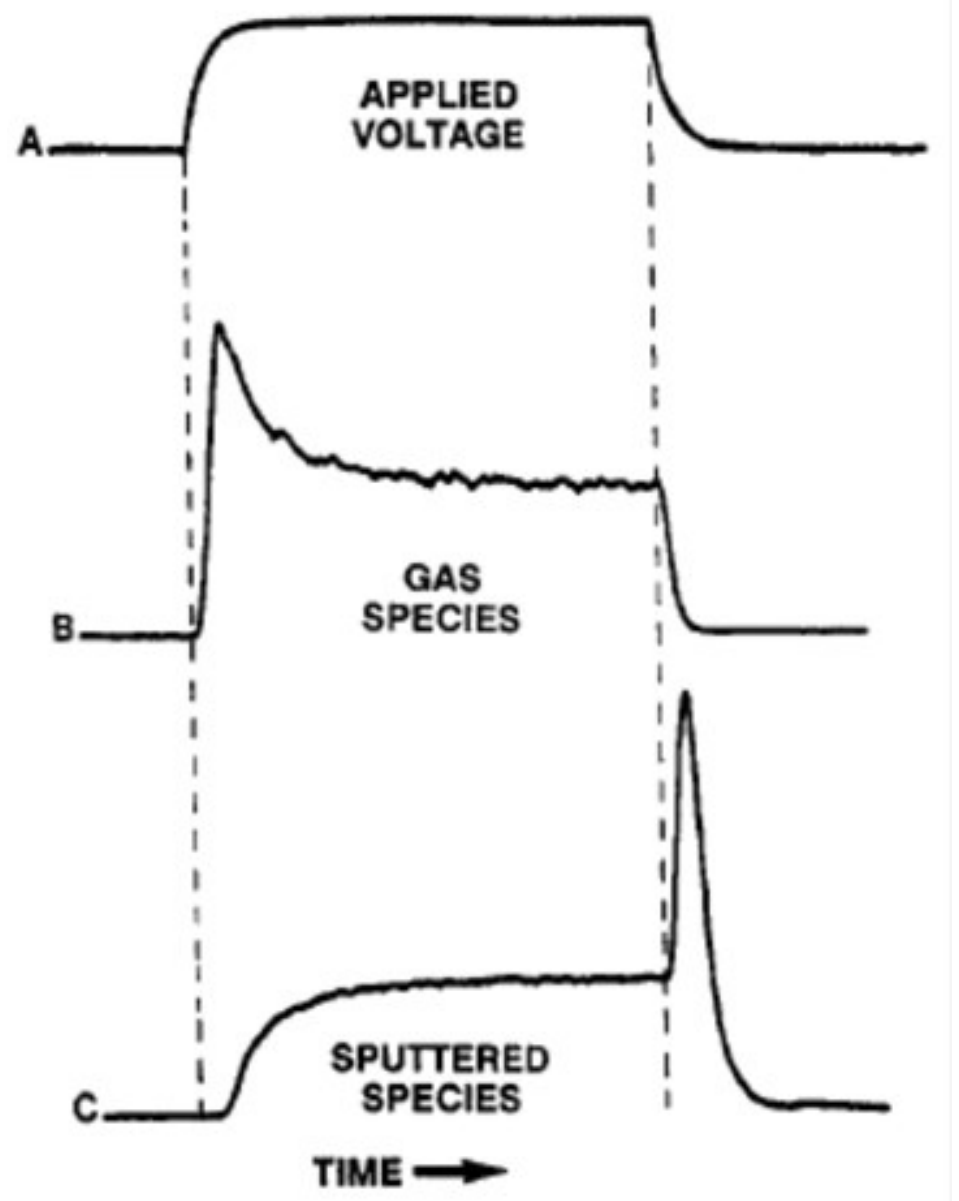

Figure 1.8 Temporal ion signal profiles in a pulsed glow discharge: (A) typical applied voltage, (B) gas species, (C) sputtered species. ${ }^{35}$ 
In summary, the ion signals of gas species and sputtered species show the abnormal temporal pattern, which do not match the anticipated square-wave shape of power pulse. The gas species signals optimize in the prepeak regime, whereas sputtered species signals opitmize in after peak regimes (Figure 1.8). Therefore, pulsed glow discharge not only can enhance the analytical signals, but also allows gas related interferences to be minimized and analytical signals to be discriminated from these interferences in afterpeak regime. For example, Lewis et al. utilize this advantage to analyze the ${ }^{40} \mathrm{Ca}^{+}$in the presence of ${ }^{40} \mathrm{Ar}+$ by pulsed glow discharge connected with a time gated detector. ${ }^{40}$

Unlike steady state glow discharge, pulsed glow discharge can offer different ionization environments for an analyte because of three different time regimes. The time regime close to prepeak provides "hard" ionization by domination of highly energetic electron collisions, which can give higher degree of fragmentation and elemental information of an analyte molecule. For the plateau regime, the ionization environment is a combination of electron ionization, charge transfer, and Penning ionization, which gives rich structural information of the molecule. During the afterpeak regime, the relatively "soft" Penning ionization may produce molecular ions and larger fragment ions for each molecule. Therefore, different time resolved mass spectra, including elemental to molecular information, can be obtained by pulsed glow discharge coupled with timeresolved mass spectrometer, such as time-of-flight mass spectrometer. ${ }^{41-43}$ 


\subsubsection{Pulsed Glow Discharge Time of Flight Mass Spectrometry (GD- TOFMS)}

A time of flight (TOF) mass analyzer is well suited to obtaining pulsed ion signals produced from pulsed glow discharge ion source. The big advantage of TOFMS is the fast analysis speed, which permits acquisition of the whole mass spectrum over a wide mass range for each glow discharge pulse. Compared to scanning analyzer, such as quadrupole and double-focusing, TOFMS decreases time for collection of the entire mass spectrum and minimizes the spectral error induced by temporal variation because of concurrent detection of ion signals..$^{28,44}$ Because of the fast analysis speed of TOFMS, a large amount of mass spectra can be obtained and averaged in a short time, lead to the enhancement of signal to noise ratio. ${ }^{45}$ In addition, TOFMS has high ion transmission efficiency. ${ }^{28}$ The inherent temporal selectivity of TOFMS enables detection of ions formed in different temporal regime during each pulse cycle. ${ }^{44}$ These characteristic advantages enable nearly concurrent acquisition of elemental, structural, and intact parent molecular information by pulsed GD-TOFMS. ${ }^{46}$ 

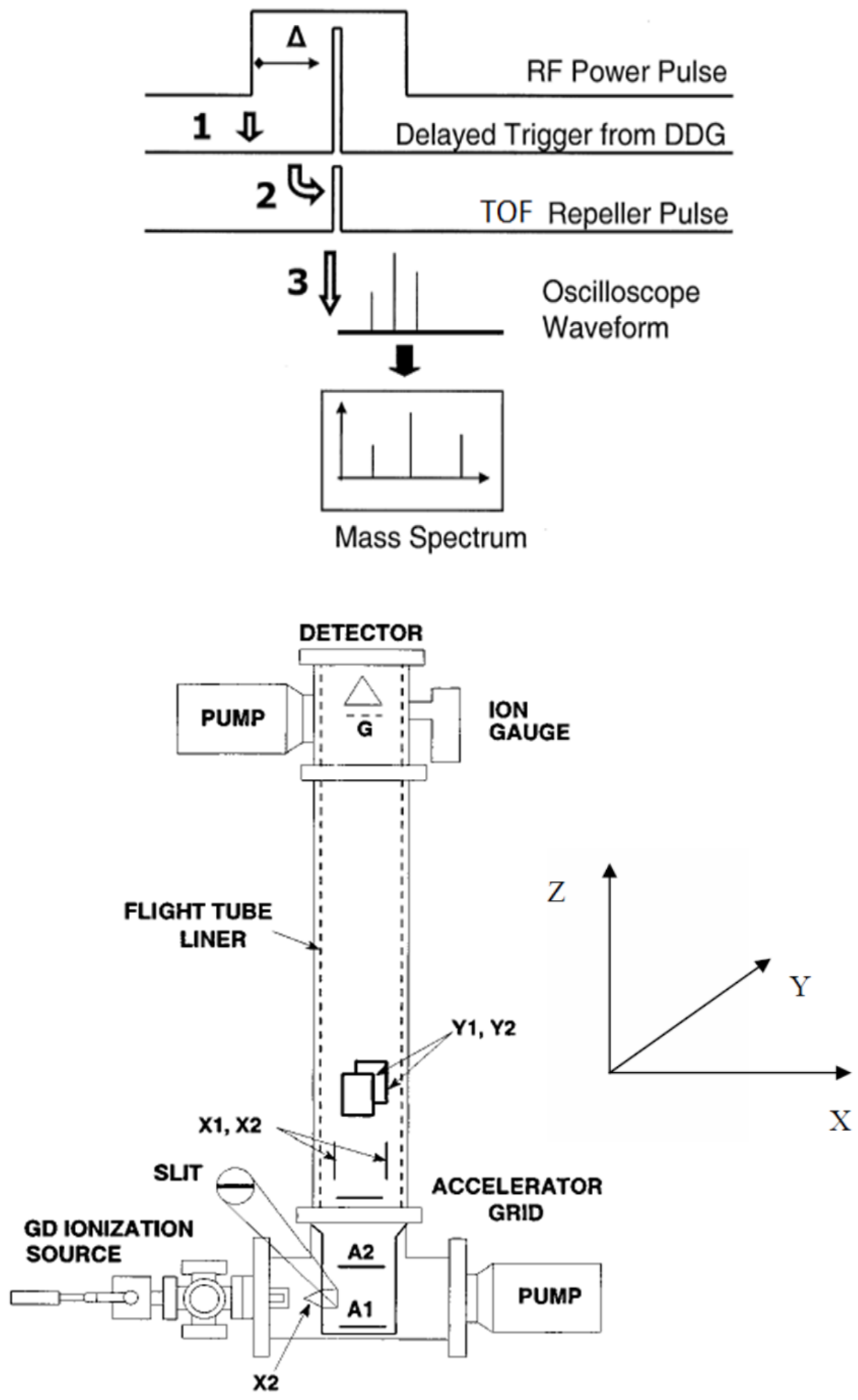

Figure 1.9 Diagram of glow discharge time of flight and the setup of data acquisition. ${ }^{44}$ 
The setup of pulsed GD-TOFMS and data acquisition is shown in Figure 1.9. The orthogonal positioning of flight tube relative to ion beam (X direction ) increases resolution, because the dispersion of kinetic energy for ions from same $\mathrm{m} / \mathrm{z}$ is reduced in flight tube direction ( $Z$ direction). ${ }^{28,44}$ The ions formed in glow discharge source pass through the skimmer and enter the extraction region of TOF. There is a slit between the skimmer and extraction region to limit the spatial distribution of ions into the TOF. After ions enters TOF in $\mathrm{X}$ direction, they are held between repeller plate $\mathrm{A} 1$ and extraction grid $\mathrm{A} 2$ until injection by pulsing the repeller positive voltage at specific time. The application of micro second pulse voltage to the repeller is triggered by a digital delay generator (DDG) synchronized with a radio frequency $(\mathrm{RF})$ power pulse, injecting the ions into the acceleration region between extraction grid A2 and accelerator grid. With application of an acceleration potential $\mathrm{V}$, the ions is accelerated in $\mathrm{Z}$ direction towards the flight tube. After passing through the strong electric field, the potential energy $E_{p}$ of ions with mass $m$ and charge $z$ convert into kinetic energy $E_{k}$ and result in the final velocity $v$ of ions.

$$
E_{k}=m v^{2} / 2=z V=E_{p}
$$

After acceleration, the final velocity of ions is:

$$
v=(2 z V / m)^{1 / 2}
$$

The velocity of ions leaving the acceleration region is inversely proportional to their mass to charge ratio $\mathrm{m} / \mathrm{z}$. The ions with smaller $\mathrm{m} / \mathrm{z}$ have greater velocity. After acceleration, the ions enters and travels through the flight tube, which is a field free region, with a constant velocity. The time $t$ needed for ions traverse the tube with length $L$ is: 


$$
t=L / V
$$

The $v$ in Equation (1-7) is substituted by Equation (1-6) and gives the equation $(1-8)$ as follows:

$$
t=L(m / z)^{1 / 2} /(2 V)^{1 / 2}
$$

The time is directly related to the mass to charge ratio $\mathrm{m} / \mathrm{z}$ of ions because of the same voltage and tube length. The ions with different $\mathrm{m} / \mathrm{z}$ can be separated base on the different traveling time. The ions with larger $\mathrm{m} / \mathrm{z}$ will travels slower in the flight tube and reach the detector later than ions with smaller $\mathrm{m} / \mathrm{z}^{28}$

The $\mathrm{m} / \mathrm{z}$ can be obtained by measurement of flight time of ions through the tube:

$$
m / z=2\left(V / L^{2}\right) t^{2}
$$

The dual multichannel plate detector located at the end of the flight tube is used to measure the flight time of ions. The mass spectrometric signal is collected by an oscilloscope. The mass spectra data are averaged and transferred to the computer to produce a complete mass spectrum. 


\subsubsection{Application of Glow Discharge Mass Spectrometry (GDMS) for}

\section{Chemical Speciation}

Chemical speciation is the process to identify and quantify the different chemical species in a sample. Chemical speciation is important in many fields like environmental, nutritional, and pharmaceutical chemistry. ${ }^{30.47}$ GDMS is a well-established technique for the direct determination of elements in solid samples (i.e. geological materials). ${ }^{27,} 48$ The introduction of pulsed glow discharge and other variation conditions (i.e. spatial variation and power) make internal energy tunable so that the chemical speciation can be performed at elemental, structural, and molecular level. This is a big advantage for GDMS used for chemical speciation..$^{39,43,49}$ Fliegel et al. analyzed gaseous species using GDMS coupled with gas chromatography (GC). They successfully showed elemental, structural, and molecular information was obtainable nearly simultaneously, and demonstrated the ability to quantify aromatics, halogenated hydrocarbons, and alcohol mixtures. ${ }^{30}$ Later on, the chemical speciation application of GDMS was extended to direct speciation of elemental oxidation state in solid state sample. At first, the elemental analysis is to identify the total concentration of the element in the sample. ${ }^{50}$ However, there is a great need for identification of oxidation state of the element because the element in different form can affect the properties of the material, such as toxicity, bioavailability, metabolism and transport. ${ }^{47,}{ }^{51-52}$ For example, hexavalent chromium $(\mathrm{Cr}(\mathrm{VI}))$ is toxic, whereas trivalent chromium $(\mathrm{Cr}(\mathrm{III})$ is an essential element needed for normal function of living organisms. ${ }^{52-53}$ GDMS has several advantages for elemental speciation. First, it is a direct elemental speciation method without sample dissolution and additional pretreatment 
procedures (i.e. extraction, derivatization, or preconcentration). This direct speciation technique not only makes the analysis fast and convenient, but also allows analysis of the solid species in their native form to avoid changing oxidation state of species. ${ }^{53-54}$ Second, GDMS separates the sputtering and ionization processes in time and space leading to a decreased matrix dependence for analytical signal and minor variations in elemental sensitivity, generating representative population of species from the original sample. ${ }^{27,} 36,52$ Previous research successfully used the specific cluster ion for direct speciation of chromium (III, VI) and manganese (II, IV) and quantify metal species in solid sample. ${ }^{52,54}$ Later on, Gu et al. demonstrate the ability to differentiation between $\mathrm{FeO}$, $\mathrm{Fe}_{2} \mathrm{O}_{3}$, and $\mathrm{Fe}_{3} \mathrm{O}_{4}$ using ion abundance ratio comparisons. ${ }^{55}$ 


\subsection{References}

1. Pan, Tao; Fiorini, Gina S.; Chiu, Daniel T.; Woolley, Adam T. In-Channel AtomTransfer Radical Polymerization of Thermoset Polyester Microfluidic Devices for Bioanalytical applications, Electrophoresis, 2007, 28, 2904-2911.

2. Wang, Y.; Stevens, A. L.; Han, J., Million-fold Preconcentration of Proteins and Peptides by Nanofluidic Filter, Analytical Chemistry, 2005, 77, 4293-4299.

3. Lee, J. H.; Chung, S.; Kim, S. J.; Han, J., Poly(dimethylsiloxane)-Based Protein Preconcentration Using a Nanogap Generated by Junction Gap Breakdown, Analytical Chemistry, 2007, 79, 6868-6873.

4. Kelly, K. C.; Miller, S. A.; Timperman, A. T., Investigation of Zone Migration in a Current Rectifying Nanofluidic/Microfluidic Analyte Concentrator, Analytical Chemistry, 2009, 81, 732-738.

5. Zhang, Y.; Timperman, A. T., Integration of Nanocapillary Arrays into Microfluidic Devices for Use as Analyte Concentrators, Analyst, 2003, 128, 537-542.

6. Kim, S. M.; Burns M. A.; Hasselbrink, E. F., Electrokinetic Protein Preconcentration Using a Simple Glass/Poly(dimethylsiloxane) Microfluidic Chip, Analytical Chemistry, $2006,78,4779-4785$.

7. Kuo, T.C.; Cannon, D. M., Jr.; Chen, Y.; Tulock, J. J.; Shannon, M. A.; Sweedler, J. V.; Bohn, P. W., Gateable Nanofluidic Interconnects for Multilayered Microfluidic Separation Systems, Analytical Chemistry, 2003, 75, 1861-1867. 
8. Gatimu, E. N.; King, T. L.; Sweedler, J. V.; Bohn, P. W., Three-Dimensional Integrated Microfluidic Architectures Enabled through Electrically Switchable Nanocapillary Array Membranes, Biomicrofluidics, 2007, 1, 21502.

9. Tulock, J. J.; Shannon, M. A.; Bohn, P. W.; Sweedler, J. V., Microfluidic Separation and Gateable Fraction Collection for Mass-Limited Samples, Analytical Chemistry , 2004, 76, 6419-6425.

10.Pu, Q.; Yun, J.; Temkin, H.; Liu, S., Ion-Enrichment and Ion-Depletion Effect of Nanochannel Structures, Nano Lett ,2004, 4, 1099-1103.

11. Plecis, A.; Schoch Reto, B.; Renaud, P., Ionic Transport Phenomena in Nanofluidics: Experimental and Theoretical Study of the Exclusion-Enrichment Effect on a Chip, Nano Lett, 2005, 5, 1147-1155.

12. Probstein, R. F., Physicochemical Hydrodynamics. An Introduction, 1989, 185-198.

13. Harris, D. Quantitative Chemical Analysis, Sixth Edition, 2003, 658.

14.Zhou, K.; Perry, J. M.; Jacobson, S.C., Transport and Sensing in Nanofluidic Devices, Annual Review of Analytical Chemistry, 2011, 4, 321-341.

15. Reschke, K.C., Development of Nanofluidic/Microfluidic Interfaces as Analyte Concentrators for Proteomic Samples, West Virginia University Libraries: Morgantown, WV, 2010.

16. Miller S.A.; Kelly K.C.; Timperman A.T., Ionic Current Rectification at a Nanofluidic/ Microfluidic Interface with an Asymmetric Microfluidic System, Lab on a Chip, 2008, 8, $1729-1732$.

17.Karnik, R.; Duan, C.; Castelino, K.; Daiguji, H.; Majumdar, A., Rectification of Ionic Current in a Nanofluidic Diode, Nano Lett, 2007, 7, 547-551. 
18. Cheng L.J.; Guo L.J., Nanofluidic Diodes, Chem. Soc. Rev., 2010, 39,923-938

19.Wang, C.; Xu, J.; Chen, H.; Xia, X., Mass Transport in Nanofluidic Devices, Science China: Chemistry, 2012, 55, 453-468.

20. Siwy, Z. S., Ion-Current Rectification in Nanopores and Nanotubes with Broken Symmetry, Adv. Funct. Mater., 2006, 16, 735-746.

21. Yusko, E.C.; An, R.; Mayer, M. 2010. Electroosmotic Flow Can Generate Ion Current Rectification in Nano- and Micropores, ACS Nano, 2010, 4,477-487.

22. Marcus, R. K., Glow Discharge Spectroscopies, Plenum Press: New York and London, 1993.

23. Marcus,R. K.; Broekaert, J.A.C., Glow Discharge Plasmas in Analytical Spectroscopy, John Wiley \& Sons Ltd, West Sussex, 2003.

24.Zhang, N., Elemental Speciation Using Pulsed Glow Discharge Time-of-Flight Mass Spectrometry, West Virginia University Libraries: Morgantown, WV, 2009.

25. Harrison, W. W.; Hess, K. R.; Marcus, R. K.; King, F. L., Glow Discharge Mass Spectrometry, Analytical Chemistry, 1986, 58, 341A-356A.

26. F. L. King and W. W. Harrison, Glow discharge Mass Spectrometry: an Introduction to the Technology and Its Utility, Mass Spectrom. Rev., 1990, 9, 285-317.

27. King, F. L.; Teng, J.; Steiner, R.E., Glow Discharge Mass Spectrometry: Trace Element Determinations in Solid Samples, Journal of Mass Spectrometry, 1995, 30, $1061-1075$.

28. Hoffmann, E.; Stroobant, V., Mass Spectrometry: Principles and Applications, 3rd Ed., John Wiley and Sons Ltd: West Sussex, 2007. 
29. Chapman, B., Glow Discharge Processes: Sputtering and Plasma Etching, John Wiley and Sons: New York, 1980.

30. Fliegel, D.; Waddell, R.; Majidi, V.; Günther, D.; Lewis, C. L., Quantification of Aromatic and Halogenated Hydrocarbons and Alcohol Mixtures at the Elemental, Structural, and Parent molecular Ion Level, Analytical Chemistry, 2005, 77, 18471852.

31. Marcus, R. K.; King, F. L., Jr.; Harrison, W. W., Hollow Cathode Plume as an Atomization/ Ionization Source for Solids Mass Spectrometry, Analytical Chemistry, 1986, 58, 972-974.

32. Smith, R. L.; Serxner, D.; Hess, K. R., Assessment of the Relative Role of Penning Ionization in Low-Pressure Glow Discharges, Analytical Chemistry, 1989,61, 11031108.

33. Pollmann, D.; Ingeneri, K.; Harrison, W. W., Comparison of Atomization and Ionization Processes in Direct Current, Radiofrequency and Microsecond Pulse Discharges. J. Anal. At. Spectrom. 1996, 11, 849-853.

34. Klingler, J.A.; Savickas, P.J.; Harrison, W.W., The Pulsed Glow Discharge as an Elemental Ion Source, Journal of the American Society for Mass Spectrometry, 1990, 1, 138-143.

35. Klingler, J. A.; Barshick, C. M.; Harrison, W. W., Factors Influencing lon Signal Profiles in Pulsed Glow Discharge Mass Spectrometry, Analytical Chemistry, 1991, 63, 25712576. 
36. Belenguer, Ph.; Ganciu, M.; Guillot, Ph.; Nelis, Th., Pulsed Glow Discharges for Analytical Applications, Spectrochimica Acta, Part B: Atomic Spectroscopy, 2009, 64B, $623-641$.

37.Pan, C.; King, F. L., Time-Resolved Studies of Ionized Sputtered Atoms in Pulsed Radio Frequency Powered Glow Discharge Mass Spectrometry, Anal. Chem., 1993, $65,3187-3193$.

38. Pan, C.; King, F. L., Ion Formation Processes in the Afterpeak Time Regime of Pulsed Glow Discharge Plasmas, J. Am. Soc. Mass Spectrom., 1993, 4, 727-732.

39. Li, L.; Millay, J. T.; Turner, J. P.; King, F. L., Millisecond Pulsed Radio Frequency Glow Discharge Time of Flight Mass Spectrometry: Temporal and Spatial Variations in Molecular Energetics, J. Am. Soc. Mass Spectrom., 2004, 15, 87-102.

40. Lewis, C. L.; Oxley, E. S.; Pan ,C. K.; Steiner, R. E.; King, F.L., Determination of ${ }^{40} \mathrm{Ca}+$ in the Presence of ${ }^{40} \mathrm{Ar}+$ : An Illustration of the Utility of Time-Gated Detection in Pulsed Glow Discharge Mass Spectrometry, Analytical Chemistry, 1999, 71, 230-234.

41. Steiner, R. E.; Lewis, C. L.; Majidi, V., Consideration of a Millisecond Pulsed Glow discharge Time-of-Flight Mass Spectrometer for Concurrent Elemental and Molecular analysis, Journal of Analytical Atomic Spectrometry, 1999, 14,1537-1541.

42. Majidi, V.; Moser, M.; Lewis, C.; Hang, W.; King, F. L., Explicit Chemical Speciation by Microsecond Pulsed Glow Discharge Time-of-Flight Mass Spectrometry: Concurrent Acquisition of Structural, Molecular and Elemental Information, Journal of Analytical Atomic Spectrometry, 2000, 15, 19-25.

43. Lewis, C. L.; Moser, M. A.; Dale Jr, D. E.; Hang, W.; Hassell, C.; King, F. L.; Majidi, V., Time-Gated Pulsed Glow Discharge: Real-Time Chemical Speciation at the 
Elemental, Structural, and Molecular Level for Gas Chromatography Time-of-Flight Mass Spectrometry, Analytical Chemistry, 2003, 75, 1983-1996.

44. Steiner, R. E.; Lewis, C. L.; King, F. L., Time-of-Flight Mass Spectrometry with a Pulsed Glow Discharge Ionization Source, Anal. Chem., 1997, 69, 1715-1721.

45. Hang, W.; Baker,C.; Smith, B. W.; Winefordner, J. D. ; Harrison, W. W., Microsecondpulsed glow discharge time-of-flight mass spectrometry: analytical advantages, J. Anal. At. Spectrom., 1997, $12,143-149$.

46. Li, L.; Barshick, C.M.; Millay, J. T.; Welty, A.V.; King, F. L., Determination of Bromine in FlameRetardant Plastics Using Pulsed Glow Discharge Mass Spectrometry, Anal. Chem., 2003, 75, 3953-3961.

47. Ure, A. M.; Davidson, C. M.; Chemical speciation in the environment, Blackie Academic \& Professional, London, 1995.

48. Teng, J.; Barshick, C. M.; Duckworth, D. C.; Morton, S. J.; Smith D. H.; King, F. L., Factors Influencing the Quantitative Determination of Trace Elements in Soils by Glow Discharge Mass Spectrometry, Appl. Spectrosc., 1995, 49, 1361-1366.

49. Ray, S. J.; Andrade, F.; Gamez, G.; McClenathan, D.; Rogers, D.; Schilling, G.; Wetzel, W.; Hieftje, G. M., Plasma-Source Mass Spectrometry for Speciation Analysis: Stateof-the-Art, Journal of Chromatography A, 2004, 1050, 3-34.

50. Kot, A.; Namiesnèik, J., Trends in Analytical Chemistry 2000, 19, 69-79

51. Cornelis, R. C.; Crews, H. M.; Caruso, J. A.; Heumann, K. G.,Handbook of Elemental Speciation: Techniques and Methodology, John Wiley and Sons, London, 2003. 
52. Robertson-Honecker, Jennifer N.; Zhang, Na; Pavkovich, Alexandria; King, F. L., Direct Chromium Speciation in Solid State Materials-a GDMS Approach, J. Anal. At. Spectrom, 2008, 23, 1508-1517.

53. Aubriet, F.; Poleunis, C.; Bertrand, P., Capabilities of Static TOF-SIMS in the Differentiation of First-Row Transition Metal Oxides, J. Mass Spectrom., 2001, 36, $641-651$.

54.Zhang, N.; King, F. L., Direct Manganese (Mn) Speciation in Solid State Materials by Pulsed Glow Discharge Time-of-Flight Mass Spectrometry, J. Anal. At. Spectrom., 2009, 24, 1489-1497.

55. Gu, G.; DeJesus, M.; King, F. L., Direct FexOy Speciation in Solid State Materials by Pulsed Millisecond Radio Frequency Glow Discharge Time-of-Flight Mass Spectrometry, J. Anal. At. Spectrom., 2011, 26, 816-821. 


\title{
Chapter 2
}

\section{Controlling the lon Current Rectification Factor of a Nanofluidic/Microfluidic Interface with Symmetric}

\section{Nanocapillary Interconnects}

\begin{abstract}
Previously lon current rectification has been confirmed in a nanofluidic/microfluidic interface with an asymmetric microfluidic/macrofluidic systems. In present work, the asymmetry of the NMI is systematically altered by varying the inner diameter of the NCM reservoir, and the current rectification factor is observed to increase as the NCM reservoir ID increases. Contributions from the change of solution resistance as a result of the change in the system asymmetry appear to play a primary role, while contributions from electroconvection in the NCM reservoir appear to have a secondary role. The data provide a new approach to tune the ion current rectification of NMls and strengthen the fundamental knowledge of how these devices function.
\end{abstract}




\subsection{Introduction}

Ion current rectification is an important process in the function of biological ion pores, and it can provide enhanced functionality to microfluidic and nanofluidic systems. Ion current rectification can be used to create fluidic diodes for fluidic circuits, and has been used in analyte enrichment, sensing, and control of ion concentration in microfluidic and nanofluidic systems. Ion current rectification produces a greater flow of current in one direction than in the opposite direction, and a current-voltage (I-V) plot exhibits the characteristic asymmetric shape of a diode. The degree of ion current rectification is evaluated by a current rectification factor, which is the ratio of forward bias current to the reverse bias current. Initially, ion current rectification was believed to require nanoscale asymmetry, and a number of reports achieved ion current rectification with conical nanochannels or asymmetric coatings. ${ }^{1-6}$ Using the same design reported here, ion current rectification has been observed in systems with symmetric nanochannels and asymmetry in fluidic channels that are connected to the opposite ends of the nanocapillaries. ${ }^{5}$ Additionally, ion current rectification has been achieved using microscale pores with different solutions on opposite side of the microscale pores. ${ }^{6}$

Conical nanopores are an example of nanofluidic elements with asymmetric geometry that can rectify ionic current. Typically, conically shaped pores have a diameter on the nanoscale on the narrow side (the "tip") and a larger diameter on the wide side (the "base"). For a conical nanopore with a charged surface, counterion transport is 
enhanced in the tip to base direction, which forms the ion current rectification. ${ }^{7-8}$ The first report of ion current rectification was achieved using a nanopipette. ${ }^{9}$ In addition, asymmetric nanochannels made like funnels also showed ion current rectification behavior and the effect of the taper angle of the nanofluidic funnel to the current rectification ratio. ${ }^{10}$ Moreover, there are several researchers who focus on how to control the ion current rectification of the conical nanopore. It has been reported that the degree of ion current rectification can be affected by the diameter of the conical pore, and ion current rectification increases with decreasing tip diameter. ${ }^{11}$ Moreover, several methods have been reported that use surface modification and/or $\mathrm{pH}$ adjustment to tune ion current rectification. These studies show that surface charge is an important parameter to rectify ion current. For example, ion current rectification is not observed when the internal surface becomes neutral by changing $\mathrm{pH}$ or by modifying the surface. Changing the sign for the surface form negative to positive has been shown to reverse the direction of the ion current rectification. ${ }^{8,12-16}$ Ali et al showed layer-by-layer deposition of polyelectrolytes into a conical pore to tune the ion current rectification ratio. ${ }^{17}$ In another example, the conical pore showed $\mathrm{pH}$-tunable rectifying behavior after coating with $\mathrm{pH}$-responsive polymer brush. ${ }^{18}$ Also, gold conical nanotubes are modified with DNA to control the ion current rectification. ${ }^{19}$

In addition to using asymmetric geometry, nanochannels with asymmetric surface charge were used to rectify current and form nanofluidic diode. Daiguji et al modeled a nanochannel with asymmetric or discontinuous surface charge and determined that it would perform as a fluidic diode and rectify ionic current. ${ }^{20}$ To produce surface charge discontinuity, Karnik et al modified the nanochannel surface using the diffusion limited 
patterning (DLP). Half of the nanochannel was coated with biotin which gives a neutral charge on the surface, and another half of the nanochannel had a positive charge due to the coverage with avidin. Ion current rectification was formed by coupling this chemically modified nanochannel with microchannels on either side. ${ }^{21}$ Instead of surface modification, ion current rectification was obtained by production of a $\mathrm{SiO}_{2}-\mathrm{Al}_{2} \mathrm{O}_{3}$ heterostructured nanotube which can show opposite charge between two ends of nanochannel at suitable $\mathrm{pH}^{22}$ To further improve the control of ion current rectification and ion transport, an external field was applied to change the local surface charge density of the nanofluidic diode..$^{23-26}$ For example, Guan et al introduced a single asymmetrically placed gate electrode or dual split-gate electrodes onto the top of the nanochannel to form the field-effect reconfigurable nanofluidic diodes. ${ }^{24}$ Because of asymmetric field effect, the symmetric nanochannel can form ion current rectification, and both the direction and degree of ion current rectification can be controlled by gate potential. Also, this diode can control the direction and magnitude of ion transport. Moreover, Vlassiouk and Siwy used the combination of geometric asymmetry and surface charge asymmetry to enhance ion current rectification of a conical pore..$^{27}$ In addition, the ion current rectification was reported with a nanochannel with asymmetric concentration along the channel. ${ }^{28}$

In addition to ion current rectification in nanopores introduced above, ion current rectification has also been observed in microscope conical pores with a heterogeneous liquid system. Mayer's group demonstrated that ion current rectification can be produced by using micropore with heterogeneous solutions into and out of the "tip" of conical pore. ${ }^{6}$ Two sides of the tip of the conical pore were filled by solutions with different conductance. 
When voltage applied, high-conductive solution was driven by electroosmotic flow (EOF) through the "tip" $(2.2 \mu \mathrm{m})$ of the conical pore to form high current, and low current was formed when low conductive solution was driven through the tip. Our group also built a current rectifying system using an uncoated nanocapillary membrane (NCM) with symmetric nanochannels to couple a microchannel and a larger reservoir that have an asymmetric microfluidic system. ${ }^{5}$ The nanopore shows ion permselectivity when its size is comparable to the double layer thickness. When a voltage is applied through the nanopore, concentration polarization (CP) occurs due to the exclusion of co-ions. This creates an ion-depletion zone and an ion-enrichment zone on opposing sides of the nanopores. ${ }^{29}$

Previously, we reported ion current rectification in nanofluidic/microfluidic interfaces (NMIs) with symmetric nanocapillaries, confirming that ion current rectification is not exclusively the result of nanoscale asymmetry. In these systems, the larger microfluidic/macrofluidic systems that the nanocapillaries are connected are asymmetric in size. Additionally, the cation permselectivity of the NCM causes concentration polarization (CP) enriched and depleted zones to form on opposite sides of the nanocapillaries. ${ }^{30}$ The asymmetry of the fluidic system is proposed to alter the length and stability of the CP depleted and enriched zones. ${ }^{5}$ The low current or "off" state is observed when the CP depleted zone is in the microchannel and the high current or "on" state is observed when the CP enriched zone is in the microchannel. The current rectification factor is equal to the high or "on" state current divided by the low or "off" state current observed when voltages of opposite polarity but same magnitude are applied. Herein, the asymmetry of the NMI is systematically altered by varying the inner diameter (ID) of 
the NCM reservoir, and the current rectification factor is observed to increase as the NCM reservoir ID increases. Contributions from the change of solution resistance as a result of the change in the system asymmetry appear to play a primary role, while contributions from electroconvection in the NCM reservoir appear to have a secondary role. The data provide a new approach to tune the ion current rectification of NMls and strengthen the fundamental knowledge of how these devices function.

\subsection{Experimental}

\subsubsection{Reagents and Materials}

SU -8 25 photoresist and SU-8 developer were purchased from MicoChem Corp. (Newton, MA). Tridecafluoro-1, 1, 2, 2-tetrahydrooctyl-1-trichlorosilane (United Chemical Technologies, Bristol, PA) was used as a silanizing agent to prevent the PDMS sticking to surface of silicon master. Sylgard 184 including PDMS prepolymer and the curing agent was obtained from Dow corning Corporation (Midland, MI).

Sodium phosphate electrolyte solution (10mM, $\mathrm{pH} 7.2)$ was made from sodium phosphate monobasic monohydrate (Fisher Scientific, Fair Lawn, New Jersey) and sodium phosphate dibasic heptahydrate (Fisher Scientific Fair Lawn, New Jersey). Ultra-

pure water (18.3 M $\Omega-\mathrm{cm})$ was obtained from Barnstead Nanopure Infinity Base Unit (Dubuque, IA). 
Silicon wafer with a $10 \mathrm{~cm}$ diameter was purchased from Addison Engineering, Inc. (San Jose, CA). The track-etched polycarbonate (TE-PC ) NCMs with 10nm pores (GE Osmonics Inc., Minnetonka, MN) were used to fabricate NMI based microfluidic devices.

\subsubsection{Device Fabrication}

In this study, the microfluidic/nanofluidic interfaces were formed with PDMS microfluidic devices and NCMs were used to provide the nanochannels. The microfluidic devices were designed such that the one (inlet) side of the device was similar in all cases while the inner diameter (ID) of the reservoir adjacent to the NCM (NCM reservoir) was varied on the other outlet side of the device. There were two basic microfluidic designs used in this work: one is symmetric device as shown in Figure 2.1a, and the other design is asymmetric as shown in Figure $2.1 \mathrm{~b}$. The symmetric device has similar microfluidic channels on both sides of the NCM. The horizontal channels are $100 \mu \mathrm{m}$ in width and 40 $\mu \mathrm{m}$ in depth, and vertical channels have a $150 \mu \mathrm{m}$ ID and $100 \mu \mathrm{m}$ height. The asymmetric devices have a microfluidic inlet channel with the same dimensions as the symmetric device; however, the NCM reservoir has a greater dimension than the inlet side as shown

in Figure 2.1b. A series of devices was fabricated with NCM reservoir having IDs of 150 $\mu \mathrm{m}, 300 \mu \mathrm{m}, 625 \mu \mathrm{m}$ and $850 \mu \mathrm{m}$ and heights of $\sim 1 \mathrm{~cm}$. In these devices, the critical dimension (CD) is equal to the NCM reservoir ID. 
(a)

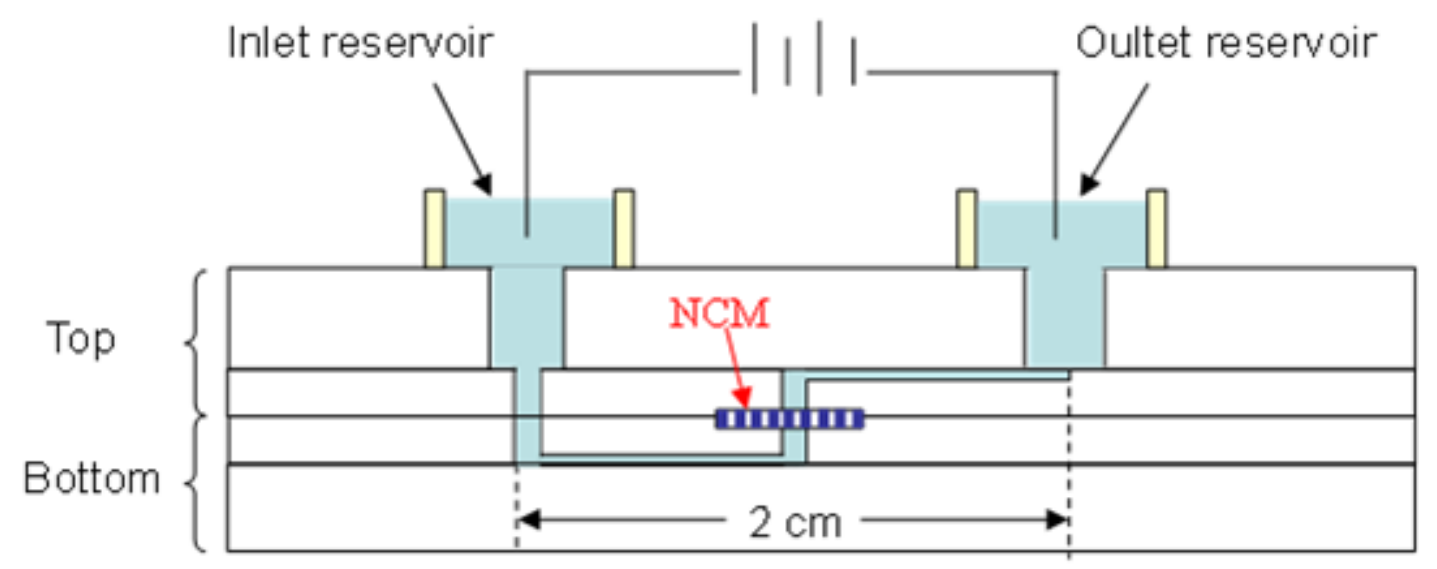

(b)

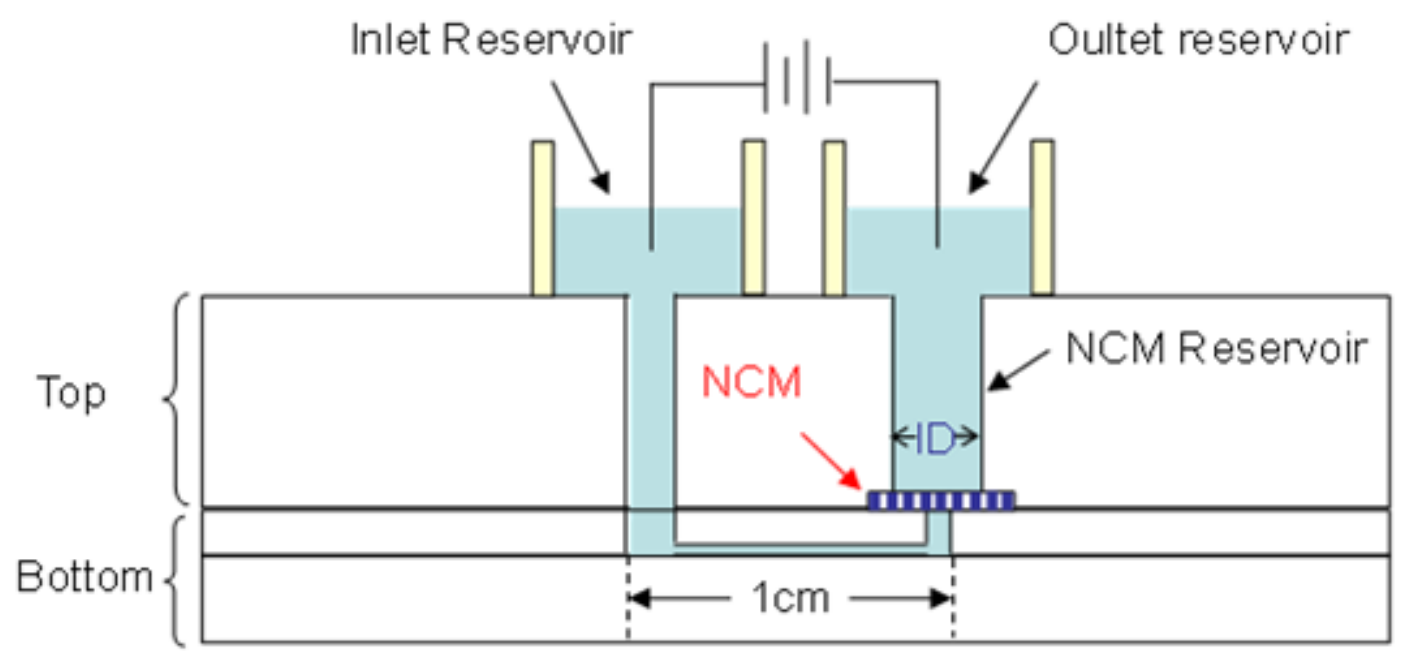

Figure 2.1 Schematic diagram of PDMS microfluidic devices. (a) Design of symmetric device. (b) Design of asymmetric device. The ID of NCM reservoir of asymmetric device varies in $150 \mu \mathrm{m}, 300 \mu \mathrm{m}, 625 \mu \mathrm{m}$ and $850 \mu \mathrm{m}$. 
The fabrication procedure of NCM based NMI devices shown in Figure 2.1 included three main steps as described before. ${ }^{30}$ First, 3-D masters used in this work were fabricated from a $10 \mathrm{~cm}$ diameter silicon wafer (Addison Engineering, Inc., San Jose, CA) using SU 8-25 photoresist. Two layers were made to make 3-D photoresist master using photolithography technique. The second layer was made by repeating the photolithography process following the completion of first layer fabrication. The pattern of first layer was used to make the horizontal channels, and the posts on the second layer were used to make vertical channels with $100 \mu \mathrm{m}$ height. At first, the photoresist was spin coated at $1000 \mathrm{rpm}$ for $30 \mathrm{~s}$ to silicon wafer and pre-baked on the hot plate at $90^{\circ} \mathrm{C}$ for 20 mins. After that, the photoresist coated silicon wafer was covered by the mask, exposed under UV-light $(365 \mathrm{~nm})$ for $30 \mathrm{~s}$, and post-baked at $90{ }^{\circ} \mathrm{C}$ for $10 \mathrm{mins}$. Finally, the unexposed part was removed by immersing the wafer in SU-8 developer for 3 mins. The second layer was made by spin coated at $500 \mathrm{rpm}$ for $15 \mathrm{~s}$ and pre-baked at $90{ }^{\circ} \mathrm{C}$ for 2 hours. After that, the wafer was cooled to the temperature and exposed under UVlight for $220 \mathrm{~s}$. Then the coated wafer was developed in SU-8 developer for 6 mins. After coating of two layers photoresist to the wafer, the $3 \mathrm{D}$ master is hard baked at $150{ }^{\circ} \mathrm{C}$ for 30mins. Then the master surface was treated with tridecafluoro-1,1,2,2-tetrahydrooctyl1-trichlorosilane under the vacuum for $1 \mathrm{~h}$. Second, the PDMS pieces were made separately to fabricate the devices shown in Figure 2.1. A 10:1 mixture of PDMS prepolymer and the curing agent was degassed in a vacuum desiccator (Bel-art products, Pequannock, New Jersey) to remove all the bubbles and poured to suitable master molds to make PDMS pieces. To make the $100 \mu \mathrm{m}$ patterned layer of the PDMS (with horizontal 
channel and $100 \mu \mathrm{m}$ vertical channels on it), the PDMS mixture on the 3D silicon master was spin coated at $1000 \mathrm{rpm}$ for $30 \mathrm{~s}$ and cured on a hotplate at $90^{\circ} \mathrm{C}$ for 20 mins. The thick top and bottom layers were also made with PDMS by a plexiglass machined mold. The thick layers were cured at $70{ }^{\circ} \mathrm{C}$ for 2 hours. Finally, the top and bottom parts of PDMS pieces were plasma treated using a PDC-32G surface Plasma Cleaner (Harrick Scientific, Ossining, NY) and irreversibly bonded together with the NCM sandwiched in the middle as shown in Figure 2.1.

\subsubsection{Current Measurements}

Before each current measurement experiment, the microchannel and reservoirs were filled fresh $10 \mathrm{mM}$ sodium phosphate electrolyte solution at $\mathrm{pH}$ 7.2. Inlet reservoir and outlet reservoir were filled with $1 \mathrm{~mL}$ phosphate buffer to make solution levels of two reservoirs equal. After each measurement, the solutions in the reservoirs were tested with $\mathrm{pH}$ paper to ensure the $\mathrm{pH}$ did not drift. To supply the electrical contact to the microfluidic channel, the platinum electrodes were placed in the reservoirs. In addition, the reservoirs are covered to minimize solution evaporation. A High Voltage Power Supply (TREK Model 610E) was used to apply potential to each reservoir through a program written by National Instruments Lab Windows/CVI 8.0. There were three voltage programs used in this study: One is for both symmetric and asymmetric devices the without NCM $(150,150,-150,-100,-50,0,10,50,100,150$ each 5 mins); Second is for symmetric devices with NCM ( -150V,-150V, -150V, -150V, -150V $-125 \mathrm{~V},-100 \mathrm{~V},-75 \mathrm{~V},-$ 
$50 \mathrm{~V},-10 \mathrm{~V}, 0 \mathrm{~V}, 0 \mathrm{~V}, 150 \mathrm{~V}, 150 \mathrm{~V}, 150 \mathrm{~V}, 150 \mathrm{~V}, 150 \mathrm{~V}, 125 \mathrm{~V}, 100 \mathrm{~V}, 75 \mathrm{~V}, 50 \mathrm{~V}, 10 \mathrm{~V}$ each

5 mins); The other is for the for asymmetric devices with NCM (similar to the second one except applying voltage at $150 \mathrm{~V} 50 \mathrm{mins})$. The longer times were used for larger voltages $(+/-150 \mathrm{~V})$ to ensure the time is long enough so that the current reach the quasi-steady state. Both negative voltages and positive voltages in the program were applied to the outlet reservoir. The current through the microfluidic channel was determined by measuring the voltage drop across the $10 \mathrm{k} \Omega$ resistor that was between the outlet reservoir and ground. The voltage was recorded using a digital multimeter (Agilent U1251A, Santa Clara, CA) connected with PC and Agilent GUI Data Logger software. The measured voltage values were converted to current using Ohm's law. The average quasi-steady currents of 5 runs for each voltage were extracted from the current-time (It) plots to create the current voltage plots (I-V).

\subsection{Results and Discussion}

Ion current rectification has been confirmed in a NMI fabricated with a NCM which has symmetric nanocapillaries that are nearly cylindrical. Ion current rectification is observed when there is asymmetry in the fluidic system to which the nanochannels are connected. In this study, the nanocapillaries are connected to a microfluidic channel on one side, and a larger solution reservoir on the opposite side of the NCM. To study its role in current rectification, the geometric asymmetry of the system on the current 
rectification factor, the NCM reservoir ID is altered while the microchannel dimensions are held constant.

\subsubsection{Discussion of I-V plots of Symmetric Devices and Asymmetric}

\section{Devices}

A first control uses an asymmetric microfluidic device with no NCM. Based on the $\mathrm{I}-\mathrm{V}$ plot shown in Figure 2.2b, this device provides an Ohmic response and, as anticipated, ion current rectification is not observed. In this device, without the cation permselective NCM, CP enriched and depleted zones are not present. The second control uses a symmetric device with NCM centered between two identical microfluidic channels as shown in Figure 2.1a. As shown in Figure 2.2a, the reduced current is expected because the NCM causes formation of the CP depleted zone, and no current rectification is observed because the CP depleted zone is contained in an identical microchannel regardless of the bias of the applied voltage. Compared with the asymmetric microfluidic device with no NCM, the current is greatly reduced and the I-V response is symmetric in shape. For all the asymmetric devices with NCM, the current is much higher when positive voltage is applied to the NCM reservoir (the "on" state) as compared to negative voltage (the "off" state), producing an asymmetric I-V plot, known as ion current rectification. The degree of current rectification increases with larger ID of NCM reservoir. 
(a)

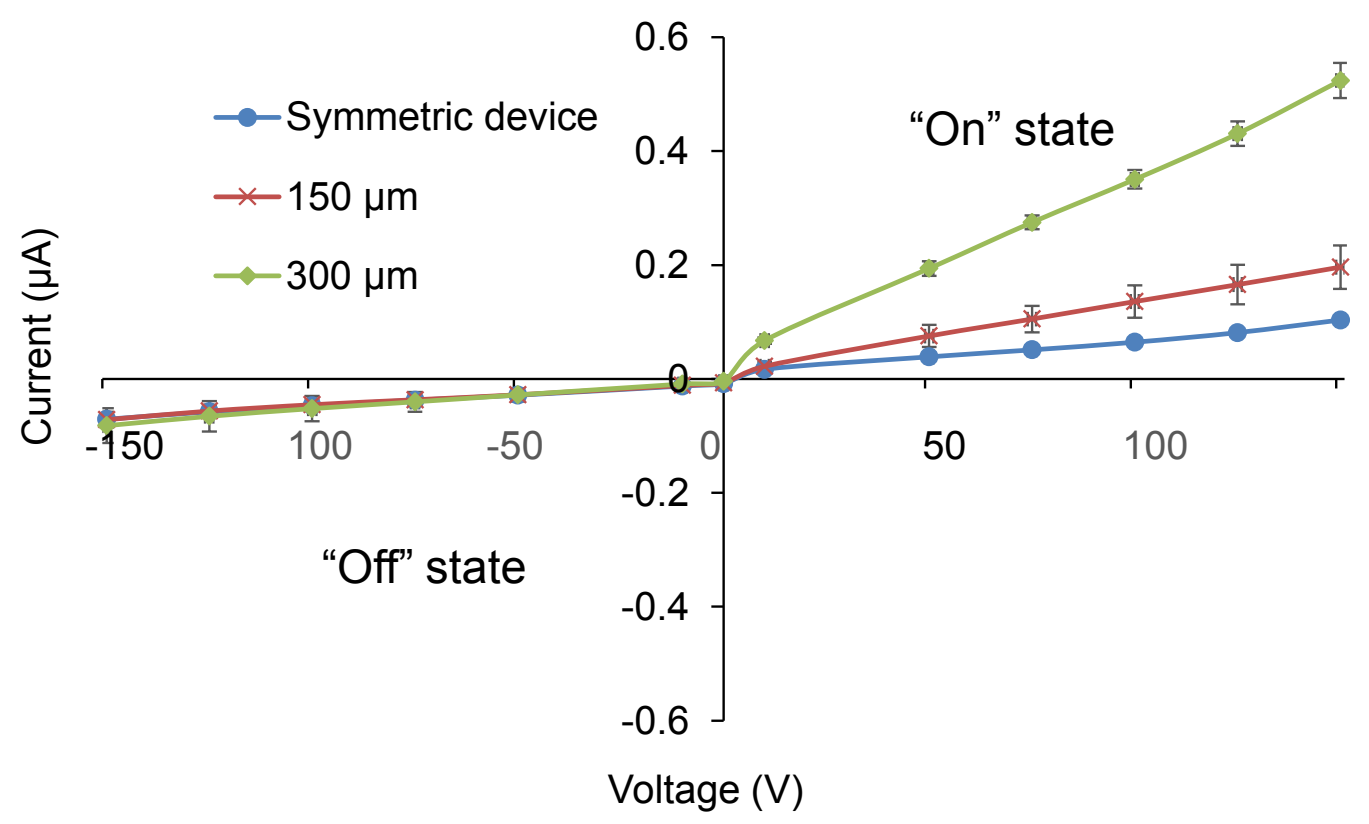

(b)

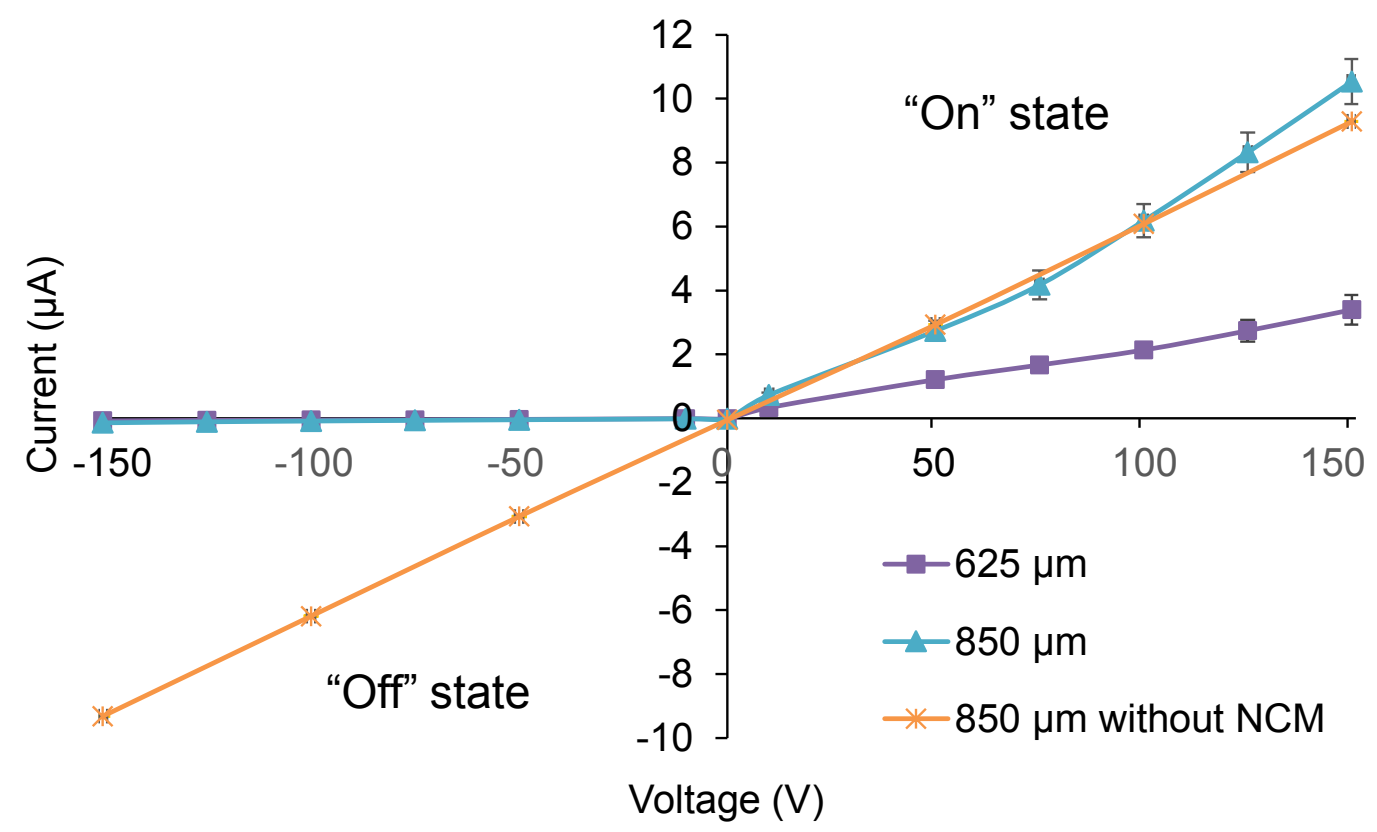

Figure 2.2 I-V plots for symmetric device and asymmetric devices with different NCM reservoir ID. Each data point is the average quasi-steady currents of 5 runs for each voltage were extracted from the current-time (I-t) plots to build the current voltage plots $(\mathrm{I}-\mathrm{V})$. 


\subsubsection{Effect of Inner Diameter of NCM Reservoir on Ion Current Rectification}

Factor

The degree of ion current rectification is evaluated by a current rectification factor, which is the ratio of the "on" state current to the "off" state current observed when voltages of opposite polarity but same magnitude are applied. As shown in Figure 2.3 and Table 2.1, increasing the NCM reservoir ID increases the current rectification factor with the same voltage applied. The device with the least degree of asymmetry has a $150 \mu \mathrm{m} \mathrm{ID}$ NCM reservoir and yields a rectification factor of 2.7. As the NCM reservoir ID is increased to $300 \mu \mathrm{m}$, the rectification factor increases to 6.4 ; and as the NCM reservoir ID is increased to $625 \mu \mathrm{m}$, the rectification factor increases to 44 . The device with the largest NCM reservoir, with an ID of $850 \mu \mathrm{m}$, produced the largest rectification factor of 75. From Table 2.1, it can be noted that the increase in the rectification factors follows the increase in the cross-sectional area.

Table 2.1 Cross-sectional area of NCM reservoir and current rectification factor at \pm 150 $V$ for asymmetric devices with different ID of NCM reservoir.

\begin{tabular}{|c|c|c|}
\hline ID $(\mu \mathrm{m})$ & Cross-sectional area $\left(\mu \mathrm{m}^{2}\right)$ & Current rectification factor \\
\hline 150 & 17600 & 2.7 \\
\hline 300 & 70600 & 6.4 \\
\hline 625 & 307000 & 44 \\
\hline 850 & 567000 & 75 \\
\hline
\end{tabular}




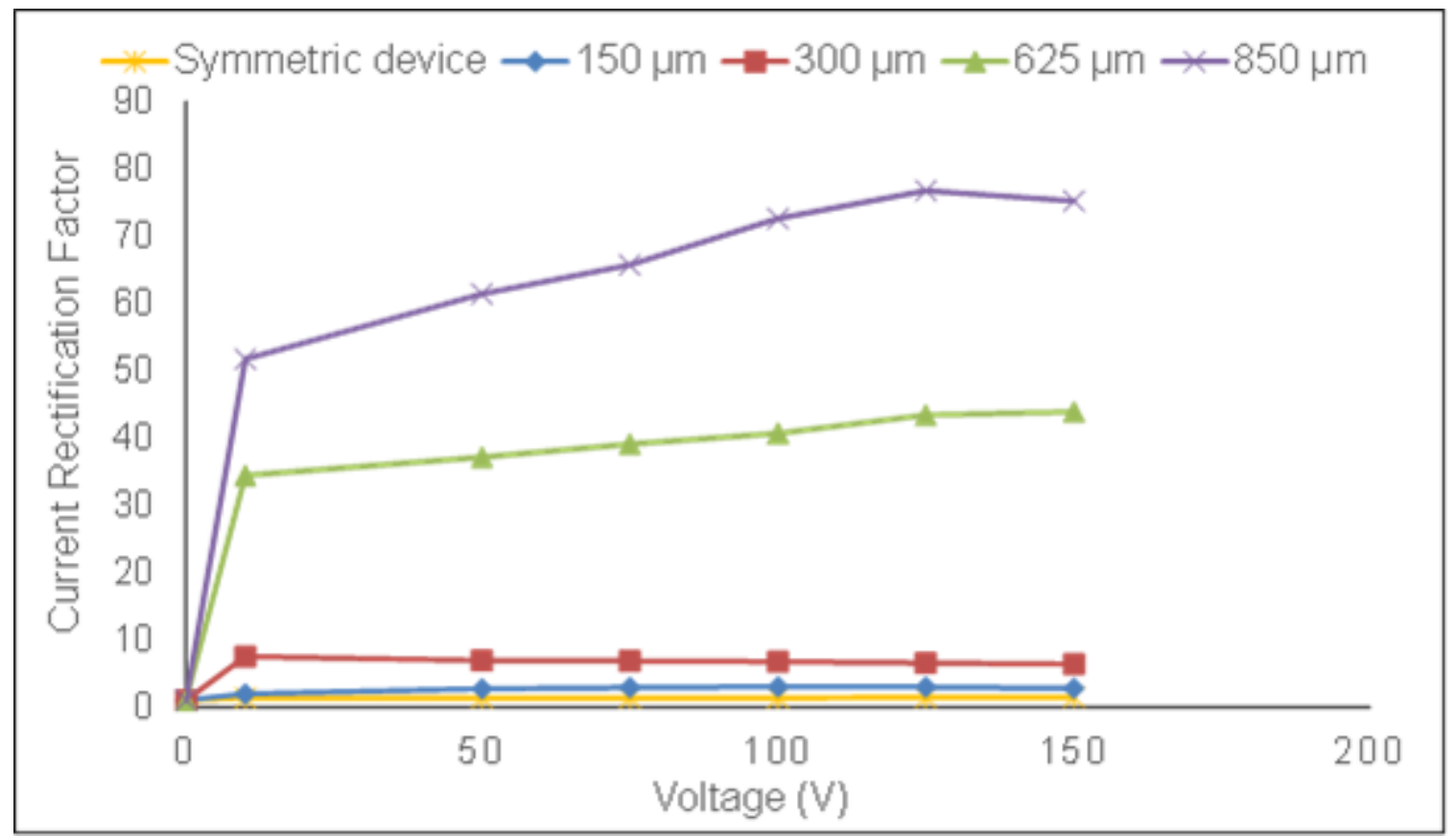

Figure 2.3 Current rectification factor versus voltage for symmetric and asymmetric devices with different ID of NCM reservoir.

Two mechanisms are likely to contribute to the ion current rectification, from the asymmetry of the larger fluidic system: a decrease in the solution resistance is possibly caused by the increase in the cross-sectional area of the NCM reservoir, and the increase of electroconvection in the NCM reservoir.

Three important fluidic regions exist in this system: the microchannel, the nanocapillaries, and the NCM reservoir. The resistance of each fluidic region is related 
to its cross-sectional area when it is filled with a homogenous solution by the following equation:

$$
R=L / A \sigma
$$

Where $R$ is the resistance, $L$ is the reservoir length, $A$ is the cross-sectional area, and $\sigma$ is the conductivity. Because the different regions of the fluidic system are in series, the total resistance is simply the sum of the resistances of different fluidic regions. If any region has a very large resistance, it will dominate the resistance of the entire system. The application of the voltage to the system causes the formation of $\mathrm{CP}$ enriched and depleted zones, as ionic current passes through the cation permselective nanocapillaries. In this system, the ion depleted zone is a region with larger resistance because of it has a lower ion concentration. Figure 2.4 shows $\mathrm{CP}$ when voltages of opposite polarity are applied. When a negative voltage is applied to the NCM reservoir, the CP depleted zone with low conductivity forms in the microchannel, as shown in Figure 2.4a, which has a small cross-sectional area and long length. The small cross-sectional area and long length of the low conductivity CP depleted zone, make this the current-limiting region of the system, and consequently, the "off" state or reverse bias current is observed (Figure 2.2). Conversely, when the polarity is reversed, the CP depleted zone forms in the NCM reservoir, and highly conductive CP enriched zone is in the microchannel (Figure 2.2b), and the increased "on" state currents are observed. At the same time, the CP depleted zone forms in the NCM reservoir, and as the ID of the NCM reservoir increases the crosssectional area increases and length of the CP depleted zone decreases. Consequently, the decreased resistance, arising from increased cross-sectional area, causes the "on" state current to increase. Because the microchannel size is held constant on inlet side, 
the "off" state currents are similar for the different devices with different NCM reservoir IDs. The observation that the current rectification factor increases with increased "on" state current arising from larger NCM reservoir ID, which as shown in Figure 2.2 and Table 2.1, is consistent with the conceptual model presented here.

Depleted CP zone

Enriched $\mathrm{CP}$ zone

$\square$ Bulk buffer solution

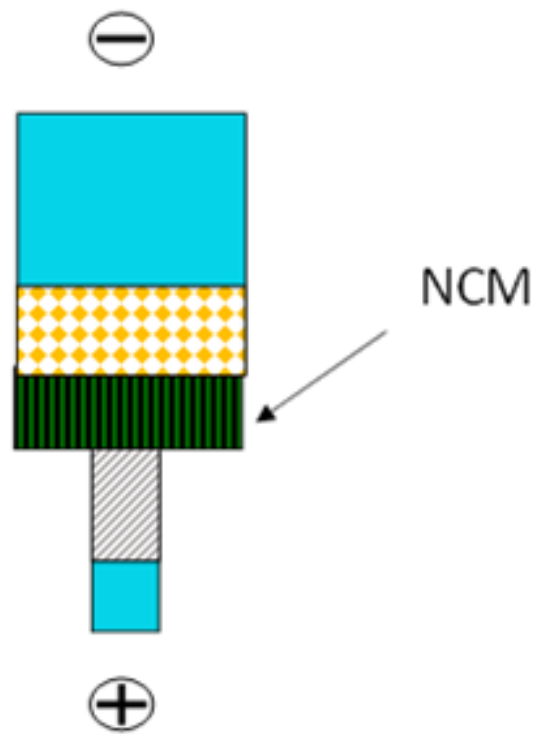

(a) "Off" state

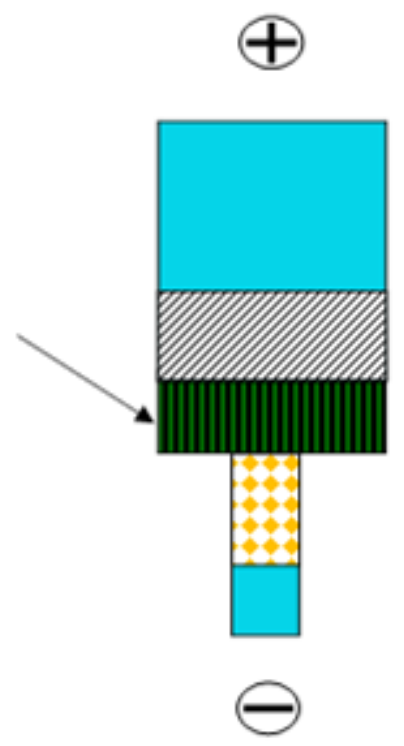

(b) On state

Figure 2.4 Schematic representation of the CP enrich zone and depleted zone at "on" state and "off" state.

In addition to the changes in cross-sectional area, the critical dimension of the NCM reservoir changes in this study. The critical dimension of the microchannel 
corresponds to the microchannel height $(100 \mu \mathrm{m}$, which is constant) and the critical dimension of the NCM reservoir corresponds to its ID. Therefore, increasing the ID of NCM reservoir while holding the dimensions of the microchannel constant also increases the difference of their critical dimensions. Increasing the critical dimension increases the propensity for convection, as described by the Reynold's number: ${ }^{32}$ The Reynold's number relates the inertial forces that induce convection to the viscous forces that stabilize the fluid, and is expressed by eqn (2-2) .

$$
\operatorname{Re}=\frac{d_{c} \rho\langle v\rangle}{\eta}
$$

Reynold's number $(R e)$ is directly related to the channel diameter $d_{c}$, fluid density $\rho$, and flow velocity $\langle U\rangle$, and inversely related to viscosity $\eta$. Convection is effectively suppressed in the microchannel because of the low Reynold's number generated by small critical dimension. While the velocity of the fluid through the system is very low, the concentration gradients that result from CP provide significant convective forces. These forces can disturb the diffusion layer and induces convective mixing of diffusion layer at higher voltage, developing the electroconvection.

The electroconvection can induce the overlimiting current, which is observed in asymmetric device with $850 \mu \mathrm{m}$ NCM reservoir, as shown in Figure 2.2b. Similar overlimiting current behavior was observed with similar NMI by Miller, et al. ${ }^{5}$ A more indepth discussion of electroconvection has been published by Rubenstein. ${ }^{33-34}$ Therefore, the NCM reservoir with larger ID of NCM reservoir have more propensity for electroconvection because of their larger Reynold's number, especially at high voltage. As shown in Figure 2.3, the current rectification factor increases with the voltage more 
obviously in the asymmetric devices with larger NCM reservoir. This is probably due to occurrence of the electroconvection in the larger NCM reservoirs (i.e. $625 \mu \mathrm{m}$ and 850 $\mu \mathrm{m})$ while the solution is effectively stabilized by the smaller reservoirs. The greater electroconvection occurred at higher voltage is due to the greater force provided by electric field. ${ }^{33-34}$ Consequently, the current rectification factor increases with voltage in NMI with larger NCM reservoir. 


\subsection{Conclusions}

In this study, the effect of the geometric asymmetry of NMls with NCM with symmetric capillaries on ion current rectification is studied by altering the NCM reservoir. It was found that current rectification factor increases as the NCM reservoir ID increases. Two mechanisms are proposed to explain this observation: one is from the decrease of solution resistance caused by the increase in the cross-sectional area of the NCM reservoir, the other is from the increase of electroconvection in the NCM reservoir. The data provide a new approach to tune the ion current rectification of NMls and strengthen the fundamental knowledge of how these devices function. 


\subsection{References}

1. Zhou, K.; Perry, J. M.; Jacobson, S.C., Transport and Sensing in Nanofluidic Devices, Annual Review of Analytical Chemistry, 2011, 4, 321-341.

2. Cheng L.J.; Guo L.J., Nanofluidic Diodes, Chem. Soc. Rev., 2010, 39,923-938

3. Wang, C.; Xu, J.; Chen, H.; Xia, X., Mass Transport in Nanofluidic Devices,

Science China: Chemistry, 2012, 55, 453-468.

4. Siwy, Z.S., Ion-Current Rectification in Nanopores and Nanotubes with Broken Symmetry, Adv. Funct. Mater., 2006, 16, 735-746.

5. Miller S.A.; Kelly K.C.; Timperman A.T., Ionic Current Rectification at a Nanofluidic/ Microfluidic Interface with an Asymmetric Microfluidic System, Lab on a Chip, 2008, 8, $1729-1732$.

6. Yusko, E.C.; An, R.; Mayer, M. 2010. Electroosmotic Flow Can Generate Ion Current Rectification in Nano- and Micropores, ACS Nano, 2010, 4,477-487.

7. Apel, P. Y.; Korchev, Y. E.; Siwy, Z.; Spohr, R.; Yoshida, M., Diode-like Single-lon Track Membrane Prepared by Electro-Stopping, Nucl. Instr. and Meth. in Phys. Res. $B, 2001,184,337-346$.

8. Siwy, Z.; Fulinski, A., Fabrication of a Synthetic Nanopore Ion Pump. Phys. Rev. Lett. 2002, 89, $198103 / 1-198103 / 4$. 
9. Wei, C.; Bard, A. J.; Feldberg, S. W., Current Rectification at Quartz Nanopipet Electrodes, Anal. Chem. 1997, 69, 4627-4633.

10. Perry, J.M.; Zhou, K.; Harms, Z.D.; Jacobson, S.C., Ion Transport in Nanofluidic Funnels, ACS Nano, 2010, 4, 3897-3902.

11. Kovarik, M. L.; Zhou, K.; Jacobson, S. C., Effect of Conical Nanopore Diameter on Ion Current Rectification, J. Phys. Chem. B, 2009, 113, 15960-15966.

12. Siwy, Z.; Apel, P.; Baur, D.; Dobrev, D.; Korchev, Y. E.; Neumann, R.; Spohr, R.; Trautmann, C.; Voss, K., Preparation of Synthetic Nanopores with Transport Properties Analogous to Biological Channels, Surf. Sci., 2003, 532-535, 1061-1066.

13. Siwy, Z.; Apel, P.; Dobrev, D.; Neumann, R.; Spohr, R.; Trautmann, C.; Voss, K., Ion Transport through Asymmetric Nanopores Prepared by lon Track Etching, Nucl. Instr. Meth. in Phys. Res. B, 2003, 208, 143-148.

14. Siwy, Z.; Heins, E.; Harrell, C. C.; Kohli, P.; Martin, C. R., Conical-Nanotube IonCurrent Rectifiers: The Role of Surface Charge, J. Am. Chem. Soc., 2004, 126, $10850-10851$.

15. Umehara, S.; Pourmand, N.; Webb, C. D.; Davis, R. W.; Yasuda, K.; Karhanek, M. Current Rectification with Poly-L-Lysine-Coated Quartz Nanopipettes, Nano Lett., 2006, 6, 2486-2492.

16. He, Y.; Gillespie, D.; Boda, D.; Vlassiouk, I.; Eisenberg, R. S.; Siwy, Z. S., Tuning Transport Properties of Nanofluidic Devices with Local Charge Inversion, J. Am. Chem. Soc., 2009, 131, 5194-5202. 
17. Ali, M.; Yameen, B.; Cervera, J.; Ramirez, P.; Neumann, R.; Ensinger, W.; Knoll, W.; Azzaroni, O., Layer-by-Layer Assembly of Polyelectrolytes into Ionic Current Rectifying Solid-State Nanopores: Insights from Theory and Experiment, J. Am. Chem. Soc., 2010, 132, 8338-8348.

18. Yameen, B.; Ali, M.; Neumann, R.; Ensinger, W.; Knoll, W.; Azzaroni, O., Single Conical Nanopores Displaying pH-Tunable Rectifying Characteristics. Manipulating Ionic Transport with Zwitterionic Polymer Brushes, J. Am. Chem. Soc., 2009, 131, 2070-2071.

19. Harrell, C. C.; Kohli, P.; Siwy, Z.; Martin, C. R., DNA-Nanotube Artificial Ion Channels, J. Am. Chem. Soc., 2004, 126,15646-15647.

20. Daiguji, H.; Oka, Y.; Shirono, K., Nanofluidic Diode and Bipolar transistor, Nano Lett., $2005,5,2274-2280$.

21. Karnik, R.; Duan, C.; Castelino, K.; Daiguji, H.; Majumdar, A.,Rectification of Ionic Current in a Nanofluidic Diode, Nano Lett., 2007, 7, 547-551.

22. Yan, R.; Liang, W.; Fan, R.; Yang, P., Nanofluidic Diodes Based on Nanotube Heterojunctions. Nano Lett., 2009, 9, 3820-3825.

23. Kalman, E. B.; Sudre, O.; Vlassiouk, I.; Siwy, Z. S., Control of Ionic Transport through Gated Single Conical Nanopores, Anal. Bioanal. Chem., 2009, 394, 413-419.

24. Guan, W.; Fan, R.; Reed, M.A., Field-Effect Reconfigurable Nanofluidic Ionic Diodes, Nat. Commun., 2011, 2, 506 (1-8). 
25. Karnik, R.; Fan, R.; Yue, M.; Li, D.; Yang, P.; Majumdar, A., Electrostatic Control of Ions and Molecules in Nanofluidic Transistors, Nano Lett., 2005, 5, 943-948.

26. Karnik, R.; Castelino, K.; Majumdar, A., Field-Effect Control of Protein Transport in a Nanofluidic Transistor Circuit. Appl. Phys. Lett., 2006, 88, 123114/1-123114/3.

27. Vlassiouk, I.; Siwy, Z. S. Nanofluidic Diode, Nano Lett., 2007, 7, 552-556.

28. Cheng, L. J.; Guo, L. J., Rectified Ion Transport through Concentration Gradient in Homogeneous Silica Nanochannels. Nano Lett., 2004, 7, 3165-3171.

29. Pu, Q.; Yun, J.; Termkin, H.; Liu, S., Ion-Enrichment and Ion-Depletion Effect of Nanochannel Structures, Nano Lett., 2004, 4, 1099-1103.

30. Kelly, K. C.; Miller, S. A.; Timperman, A. T., Investigation of Zone Migration in a Current Rectifying Nanofluidic/Microfluidic Analyte Concentrator, Analytical Chemistry, 2009, 81, 732-738.

31. Zhang, Y.; Timperman, A. T., Integration of Nanocapillary Arrays into Microfluidic Devices for Use as Analyte Concentrators, Analyst, 2003, 128, 537-542.

32. Giddings, J. C. Unified Separation Science, John Wiley \& Sons, 1991.

33. Rubinstein, I.; Zaltzman, B., Electroconvective Mechanisms in Concentration Polarization at Electrodialysis Membranes, Surface Chemistry and Electrochemistry of Membranes, 1999, 79, 591-621.

34. Rubinstein, I.; Zaltzman, B., Electro-Osmotically Induced Convection at a ChargeSelective Membrane, Advances in Fluid Mechanics IV, 2002, 32, 397-406. 


\title{
Chapter 3
}

\section{Analysis of Cysteine Using Pulsed Glow Discharge Time-of- Flight Mass Spectrometry}

\begin{abstract}
Glow discharge mass spectrometry (GDMS) is successfully used for elemental analysis and metal speciation. The application of GDMS to quantification of the amino acid cysteine was studied in this work. With the pulsed glow discharge time-of-flight mass spectrometry technique, the characteristic fragment ion of cysteine at $\mathrm{m} / \mathrm{z} 76$ is demonstrated as useful for quantitative analysis. The calibration curve for cysteine standards is obtained with this method exhibits good linearity $\left(R^{2}=0.9912\right)$.
\end{abstract}




\subsection{Introduction}

Glow discharge mass spectrometry (GDMS) is a well-established technique for the direct analysis of conductive solid samples. GDMS has also been modified to analyze nonconductive solid samples, such as soils, ceramics, glasses and polymers. ${ }^{1-4}$ For example, elemental analysis of both conductive solid (brass) and nonconductive solid (soil) were completed successfully using the radio frequency (rf)-powered GD coupled with MS. ${ }^{5}$ The desire to make GDMS a more versatile analytical technique has expanded its application to gaseous and liquid samples. ${ }^{1-3}$ One example of gas analysis by GDMS is the detection of 2, 4, 6-trinitrotoluene (TNT) in the ambient air using air as the discharge gas. ${ }^{6}$ Fliegel et al. analyzed gaseous species using GDMS coupled with gas chromatography (GC). They successfully showed elemental, structural and molecular information was obtainable within a single analysis, and demonstrated the ability to quantify aromatics, halogenated hydrocarbons, and alcohol mixtures. ${ }^{7} \mathrm{GC}$ coupled to GDMS was also used to determine selenomethionine in a biological sample. ${ }^{8}$

The first analysis of liquids by GDMS was accomplished by depositing the solution onto the cathode, evaporating the solution to dryness, and sputtering the solution residue. ${ }^{9}$ By using this method, both organic and inorganic liquid samples have been analyzed using GDMS. ${ }^{1-3}$ One such example of inorganic liquid analysis is the measurement of Pt and Ir levels in solution at the sub-picogram level by GDMS. ${ }^{10}$ Using a similar sampling method, Mason and coworkers were able to use GDMS for the 
elemental analysis of biological samples. In this study, $\mathrm{Pb}$ and $\mathrm{Pt}$ were quantitatively determined in urine samples collected from patients treated with the anti-cancer drug cisplatin. ${ }^{11}$ Mason and Milton also analyzed organic liquid samples including an amino acid-tyrosine by GDMS and obtained mass spectra containing both the molecular ion peak and fragment ion peaks. ${ }^{12}$

Later on, both organic and inorganic liquid samples were analyzed by coupling the particle beam (PB) interface with GDMS to introduce dry sample particles into the GD source. ${ }^{13-16}$ The mass spectrum of an amino acid-tryptophan showed both the molecular ion peak and fragment ion peaks, which is similar to a mass spectrum obtained from a classic El source. ${ }^{13}$ In addition, Matsumoto et al. detected various amino acids in liquid samples by applying a GD chemical ionization $(\mathrm{Cl})$ source combined with a vacuum nebulizer as a sample introduction system to connect with mass spectrometer. They used GD instead of a filament in conventional $\mathrm{Cl}$ to produce electrons to ionize the reacting reagent solvent (like water). Most mass spectra of amino acids obtained in this research were found to be similar to those obtained from conventional $\mathrm{Cl}$. They showed both $[\mathrm{M}+\mathrm{H}]^{+}$and $[\mathrm{M}-\mathrm{COOH}]^{+}$ions, and the relative abundance of these two types of ion peaks are different among a variety of amino acids. ${ }^{17}$

Lubman and coworkers introduced nebulized liquid sample into the atmospheric pressure glow discharge in helium to analyze organic samples including biological molecules, drugs and pesticides. It was found that this ionization source was "soft". A majority of the protonated molecular ion $[\mathrm{M}+\mathrm{H}]+$ was observed in the mass spectra of amino acids, such as tyrosine and tryptophan. For tyramine, only the $[\mathrm{M}+\mathrm{H}]^{+}$was shown in the mass spectrum, and quantification analysis based on this peak was performed. ${ }^{18}$ 
Cysteine is an amino acid present in many proteins and plays an important role in the biological system. It has been reported that the level of cysteine is related to various diseases. ${ }^{19-22}$ Therefore, the quantitative analysis of cysteine is very important. Secondary lon Mass Spectrometry (SIMS), similar to GDMS, is capable of analyzing a variety of amino acids including cysteine using both the $[\mathrm{M}+\mathrm{H}]^{+}$and $[\mathrm{M}-\mathrm{COOH}]^{+}$ions. However, the quantification of cysteine has not been demonstrated for SIMS. ${ }^{23}$

The present work demonstrates that the feasibility of a pulsed glow discharge timeof-flight method for cysteine analysis. It was found that the main characteristic fragment ion peak $[\mathrm{M}-\mathrm{COOH}]^{+}$for cysteine by rf-GDMS using argon as the discharge gas. The abundance of this fragment ion was much larger than that of other fragment ions, and no molecular ion was found in the mass spectrum. The use of this characteristic ion allows quantitative analysis of cysteine in solution, which could prove applicable to the quantification of cysteine in in liquid samples (such as biological fluids) with refinements in sensitivity of the technique. 


\subsection{Experimental}

\subsubsection{Sample Disk Design:}

A copper rod (Alloy 110, McMaster-Carr Supply Company, Cleveland, OH) was machined to make two designs of copper disks, which were used as the glow discharge cathode. The first design is a flat copper disk with $5 \mathrm{~mm}$ diameter and $6 \mathrm{~mm}$ height. The second design is the same size copper disk but with a $3 \mathrm{~mm}$ diameter reservoir in the center. By comparing the dried sample residue between the flat copper disk and the copper disk with the reservoir in the center, it was found that most samples were deposited on the edge for the flat copper disk after evaporation of solvent. For the sample disk with a reservoir in the center, the sample is deposited uniformly on the bottom of the reservoir, and is more confined and concentrated in the center of the disk, which facilitates better analytical performance. ${ }^{10}$ To find the suitable depth and diameter of the reservoir, different sizes of reservoirs were used to deposit the cysteine on the copper disk. It was found that it was difficult to load enough sample solution to the copper disk if the size of the reservoir is too small. If the reservoir is too deep, the sample is hard to be sputtered out, and much of the sample remains attached to the wall of the reservoir. Finally, the reservoir with a $3 \mathrm{~mm}$ diameter and $1 \mathrm{~mm}$ depth was chosen for our experiments. 


\subsubsection{Preparation of Sample Residue on the Sample Disk}

To achieve better analytical performance (i.e. reproducibility and calibration curve linearity) for analysis of cysteine by GD-MS, it is necessary to control several procedures to form a uniform and reproducible cysteine residue on the bottom of the copper disk during the sample preparation process. First, a suitable solvent for cysteine should be selected to deposit the sample onto the disk. To determine a suitable solvent, several solvents including pure water, 50/50 water/methanol and 50/50 water/acetonitrile were tested. Even though the addition of organic solvent makes the evaporation process faster, the cysteine sample cannot be deposited uniformly on the bottom surface of the copper disk by using mixed solvents. Pure water is chosen as solvent to form the homogenous sample residue on the surface. Second, evaporation rate was also very important to form a uniform sample residue on the bottom of the reservoir. It was found that some sample residue was left on the top of disk instead of the bottom of the reservoir if cysteine solution was dried in air. However, if the cysteine solution was dried in the vacuum desiccator with good sealing of connections, the evaporation of water would be fast enough (in 5 mins) to form a homogenous cysteine residue on the bottom of the copper disk. Third, the sample should be kept in the vacuum desiccator overnight to further drying to further remove water from the sample because the water may affect the formation process of analyte ions by lowering sputtering rate and quenching the formation of metastable argon atoms used to ionize the sample. If water remains in the different samples, the mass spectra of cysteine may not be reproducible. Fifth, the copper disk should be polished 
and rinsed with solvent to form a fresh and clean surface after each run because previous studies have found that the sputtering in glow discharge can change the surface of the copper disk and affect the sensitivity and reproducibility. ${ }^{25}$

To prepare cysteine residue used for analysis, the copper disks were baked for 40 mins in the oven at $110{ }^{\circ} \mathrm{C}$ before use. The copper disks were then cooled down in a vacuum desiccator (Bel-art products, Pequannock, New Jersey). $6 \mu \mathrm{L}$ cysteine standard solutions with a series of concentrations $(0 \mu \mathrm{g} / \mathrm{ml}, 10 \mu \mathrm{g} / \mathrm{ml}, 20 \mu \mathrm{g} / \mathrm{ml}, 30 \mu \mathrm{g} / \mathrm{ml}$, and 40 $\mu \mathrm{g} / \mathrm{ml}$ ) were deposited to the reservoir of copper disks separately. The cysteine standard solution was prepared by dissolving the cysteine (99\%, Acros Organics, Thermo Fisher Scientific, New Jersey, US) in deionized water (18.2 M $\Omega-\mathrm{cm}$, Barnstead Nanopure ultrapure water purification system, Waltham, MA). For the $0 \mu \mathrm{g} / \mathrm{ml}$ standard, pure deionized water was used to deposit on the copper disk. The solution loaded disks were kept in the vacuum desiccator overnight to form a cysteine residue film on the bottom of the reservoirs before analysis.

\subsubsection{Pulsed Glow Discharge TOF Mass Spectrometer}

The GD plasma was supplied by powering a $13.56 \mathrm{MHz}$ radio frequency (RF) generator (Model RF 10-S, RF Plasma Product Inc., Marlton, NJ) coupled with an automatic matching network (Model AM-10, RF Plasma Product Inc., Marlton, NJ). The applied power, pulse width, and duty cycle were controlled by this rf power supply system in internal pulsing mode. For this study, the power was set at $80 \mathrm{~W}$ while the pulse width 
was set at $2 \mathrm{~ms}$ with a duty cycle of $10 \%$. Ultra pure argon (Airgas, Randor, PA), used as glow discharge support gas, was introduced into the glow discharge chamber. A metering valve was used to control the gas pressure at 0.3 Torr, which is monitored by a thermocouple pressure gauge (Varian, Lexington, MA).

Because the small amount of cysteine residue deposited on the cathode would be sputtered away from the surface in a short period of time, it would be hard to use the characteristic peak from cysteine to adjust instrument parameters. Therefore, the characteristic peak from $\mathrm{Cu}^{+}(\mathrm{m} / \mathrm{z} 63$ and 65$)$, which is close to $\mathrm{m} / \mathrm{z} 76$, was used to adjust instrument parameters. The copper disk with cysteine residue was introduced onto the tip of the direct insertion probe (DIP) and surrounded by a MACOR shield (Accuratus Corporation Phillipsburg, NJ). Finally, the DIP was inserted into the glow discharge chamber for $15 \mathrm{mins}$ before turning on the glow discharge plasma. The distance between the copper cathode and ion exit orifice is $7 \mathrm{~mm}$. After that, the mass spectra of cysteine samples were collected every $1.217 \mathrm{~min}$ from 0.5 to approximately $11.5 \mathrm{mins}$ at $2.1 \mathrm{~ms}$ during the afterpeak regime. The average signal intensity of 3 runs for each concentration of standard was employed to create a calibration curve over the range of concentrations of cysteine standard. The mass spectrometer used in this study is a linear time-of-flight (TOF) mass spectrometer (R.M. Jordan Co., Grass Valley, CA), which is positioned orthogonally to the ion beam from glow discharge. Operation conditions for the TOF mass spectrometer are shown in Table 3.1. Further details of the TOF mass spectrometer and data collection were described previously. ${ }^{24}$ 
Table 3.1 TOF-MS operating parameters

\section{Time of Flight}

Flight path length

$1 \mathrm{~m}$

Ion lenses potential

Skimmer

$-375 V$

Extractor

$0 \mathrm{~V}$

Repeller

$+200 \mathrm{~V}$

Accelerator

$-1535 V$

Deflector (X1)

$-1875 V$

Deflector (Y1)

$-1550 \mathrm{~V}$

Detector

$-1900 \mathrm{~V}$

Vacuum conditions

Intermediate stage

$10^{-5}$ Torr

Flight tube

${ }^{10^{-6}}$ Torr 


\subsection{Results and Discussion}

\subsubsection{Characteristic Mass Spectra of Cysteine by GD-MS}

The pulsed glow discharge generates temporal variation in the ionization environment through each pulse cycle. Consequently, cysteine exhibits time dependent ion signal profiles during three different temporal regimes: the prepeak, plateau and afterpeak. Previous research found that there were no ion signals from sample within the prepeak regime, because it takes some time for sample to be sputtered and diffused into the negative glow before ionization. ${ }^{4,27-29}$ Because the prepeak regime is not analytically useful in this study, only plateau and afterpeak regimes were studied for determination of cysteine. For each temporal regime, mass spectra of blank and cysteine were collected and compared to identify the characteristic peak for cysteine. Before analysis by GDMS, the blank was prepared by evaporation of water loaded on the pure copper disk in vacuum desiccator, and droplets of the cysteine solution were dried on the cathode surface and sputtered by GD.

During the plateau regime, electron ionization, Penning ionization and charge transfer coexist in the negative glow, which can induce higher degree fragmentation compared to afterpeak regime. The ion signals of sample reach and remain at the steady state until the power termination. ${ }^{27,30}$ Fig. $3.1 \mathrm{a}$ and Fig $3.1 \mathrm{~b}$ show the mass spectra of a blank copper disk and a cooper disk with cysteine collected in the plateau regime (1.9 
ms). Both the mass spectra exhibit the ion signals corresponding to argon gas such as $\mathrm{Ar}^{2+}, \mathrm{Ar}^{+}$and $\mathrm{Ar}_{2}{ }^{+}(\mathrm{m} / \mathrm{z} 20,40$ and 80, respectively), and the copper isotopes at m/z 63 and $\mathrm{m} / \mathrm{z} 65$ are also observed. In addition, the ion signals of air composition are visible in the mass spectra, such as $\mathrm{H}_{2} \mathrm{O}^{+}(\mathrm{m} / \mathrm{z} 18)$, fragment ion $\mathrm{OH}^{+}(\mathrm{m} / \mathrm{z} 17)$ and $\mathrm{N}_{2}^{+}(\mathrm{m} / \mathrm{z} 28)$. Compared to mass spectrum of blank copper, mass spectrum of cysteine shows the primary fragment ion peak at $\mathrm{m} / \mathrm{z} 76$. Moreover, the enhancement of ion signals between $\mathrm{m} / \mathrm{z} 20$ to 30 , compared to blank, arise from fragment ions from cysteine. The fragment ions of cysteine in this range were also observed in the standard NIST mass spectrum using electron ionization (EI) (Fig. 3.1c). ${ }^{26}$ However, the peaks in this region on the Fig. $1 \mathrm{~b}$ are overlaid with the signals from air and argon gas. Therefore, it is hard to use peaks in this region for quantification of cysteine.

(a) Blank copper disk

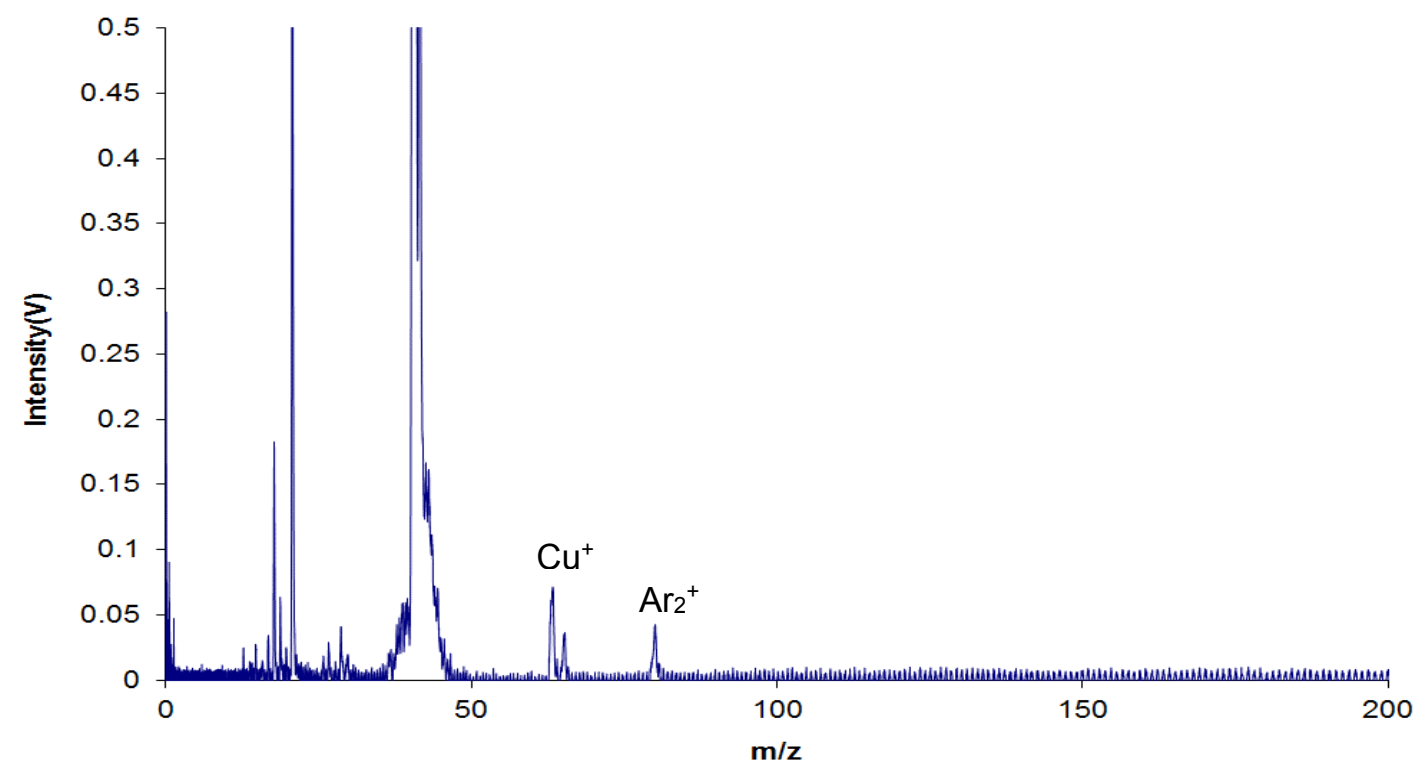


(b) Copper + Cysteine

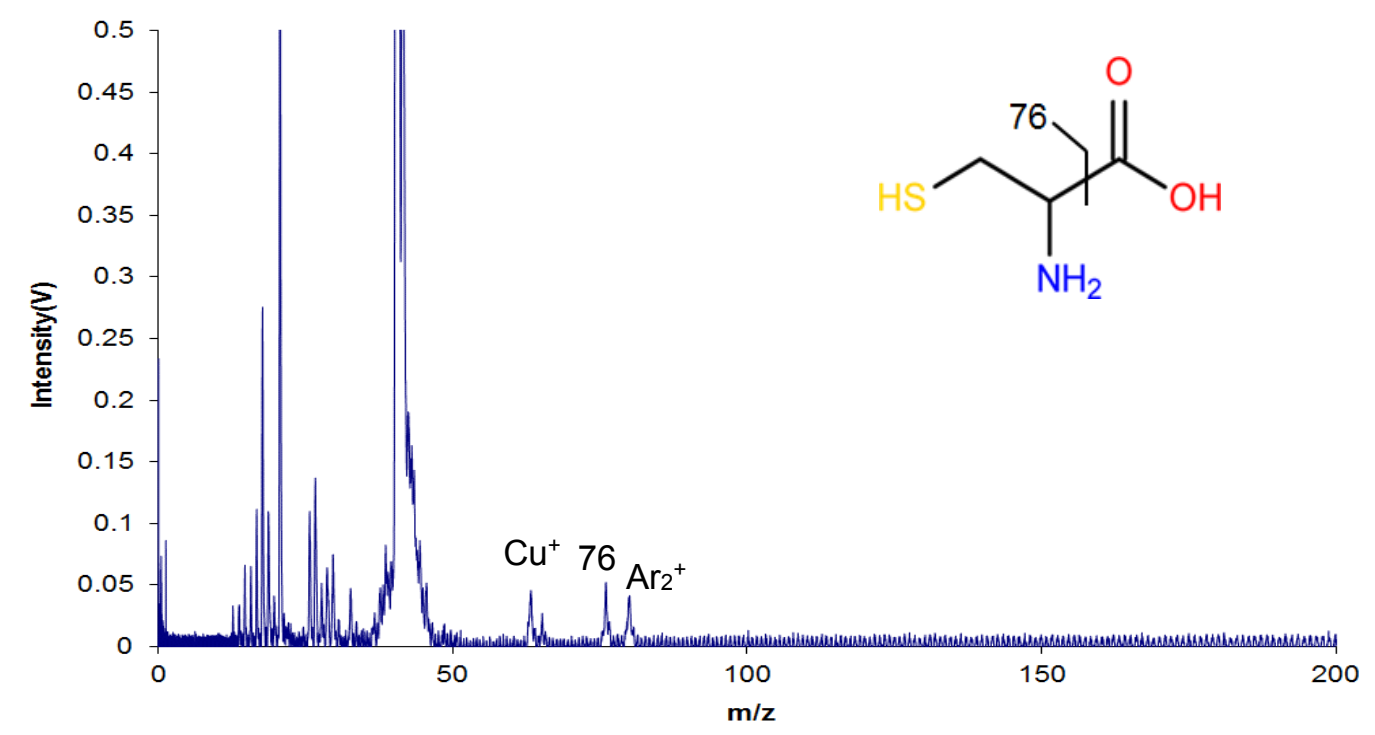

(c) Cysteine

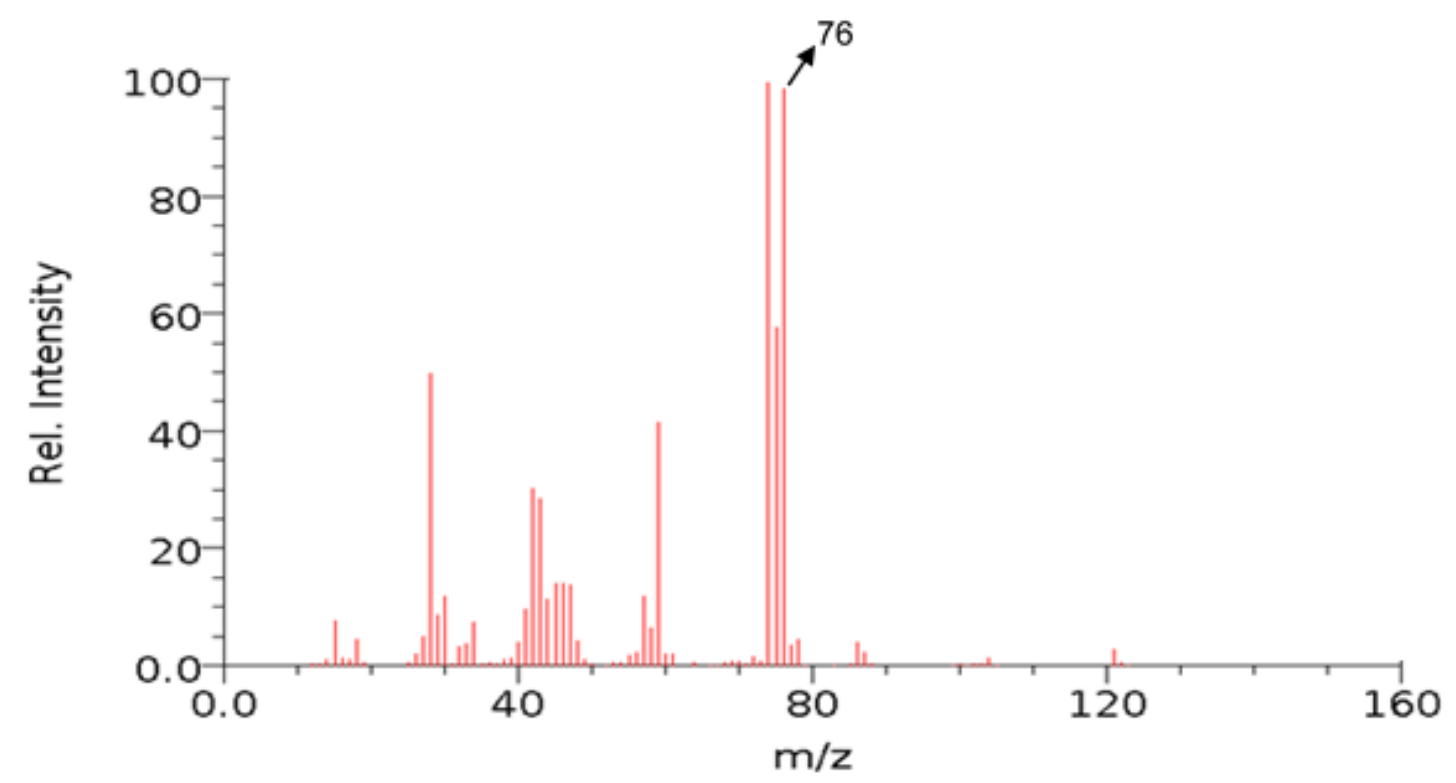

Figure 3.1 Mass spectra of (a) Blank and (b) $30 \mu \mathrm{g} / \mathrm{ml}$ cysteine during plateau regime (1.9 ms), and (c) Standard El mass spectrum of cysteine. ${ }^{26}$ 
Upon the termination of pulse power, the cathode species signals are enhanced by the increased population of metastable argon atoms, indicating the start of afterpeak regime. The surge of metastable argon atoms is induced by the recombination of thermalized electrons from collision and the argon ion. During the afterpeak, the energetic electrons lose their kinetic energy through collisional processes. Consequently, Penning ionization dominates the ionization process in this regime because of increased metastable argon atoms and decreased energetic electrons and argon ions. ${ }^{27,} 30-31$ Compared to plateau, the formation of molecular ions and larger fragment ions are preferred because Penning ionization affords relatively soft ionization compared to electron ionization and charger transfer. ${ }^{28,30}$

The mass spectra of a blank copper disk and a copper disk with cysteine collected during the afterpeak at $2.1 \mathrm{~ms}$ are shown in Figure 3.2. Both mass spectra showed the signal from copper isotopes (m/z 63 and 65) and argon dimer (m/z 80). Compared to the copper disk without cysteine, the cysteine exhibits several peaks appear between $\mathrm{m} / \mathrm{z} 50$ and 60 in the mass spectrum. These peaks may come from the fragmentation of cysteine. However, the signals of these peaks were too weak to be used for quantification. The primary fragment ion peak from cysteine is $\mathrm{m} / \mathrm{z} 76$, which is similar with that in plateau, corresponding to the loss of carboxylic acid group $(-\mathrm{COOH})$. This strong fragment ion from cysteine was also observed previously in the standard NIST mass spectrum using electron ionization (EI) and in the mass spectrum (Fig. 3.1c) obtained from SIMS experiment. ${ }^{23,26}$ This characteristic ion is then used for quantification of cysteine using GDMS. In addition, the secondary ion mass spectrum of cysteine also showed molecular 
ion peak $[\mathrm{M}+1]^{+}(\mathrm{m} / \mathrm{z} 122)$, which was not observed by GDMS in our study, probably due to the different sample preparation method and ionization process. Instead of drying cysteine solution in the reservoir of a copper cathode, they dipped a foil of silver into cysteine-water solution to form a thin non-uniform layer on the surface. ${ }^{26}$ Compared to plateau, mass spectrum in afterpeak exhibits higher intensity of peak at $\mathrm{m} / \mathrm{z} 76$. Therefore, the quantification of cysteine was performed in afterpeak regime.

(a) Blank copper disk

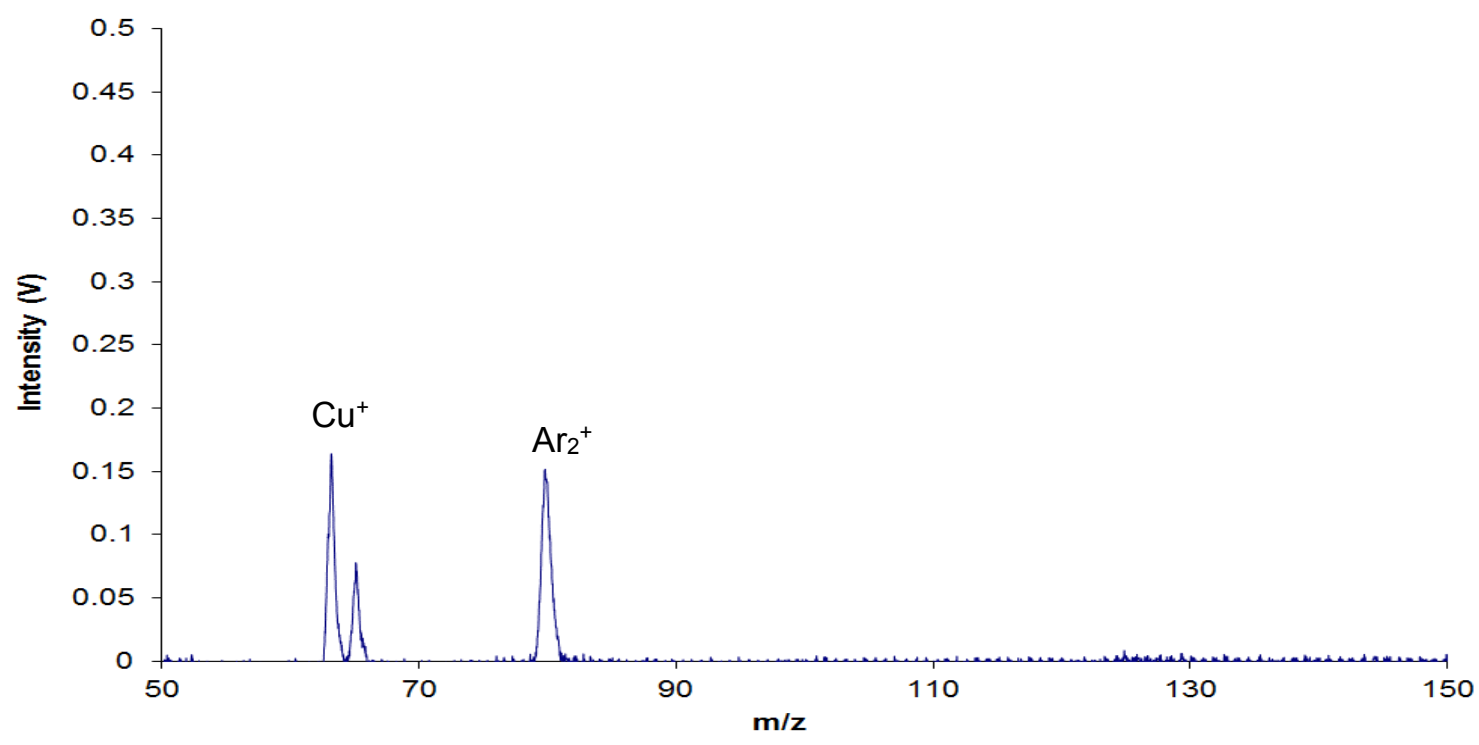


(b) Copper + Cysteine

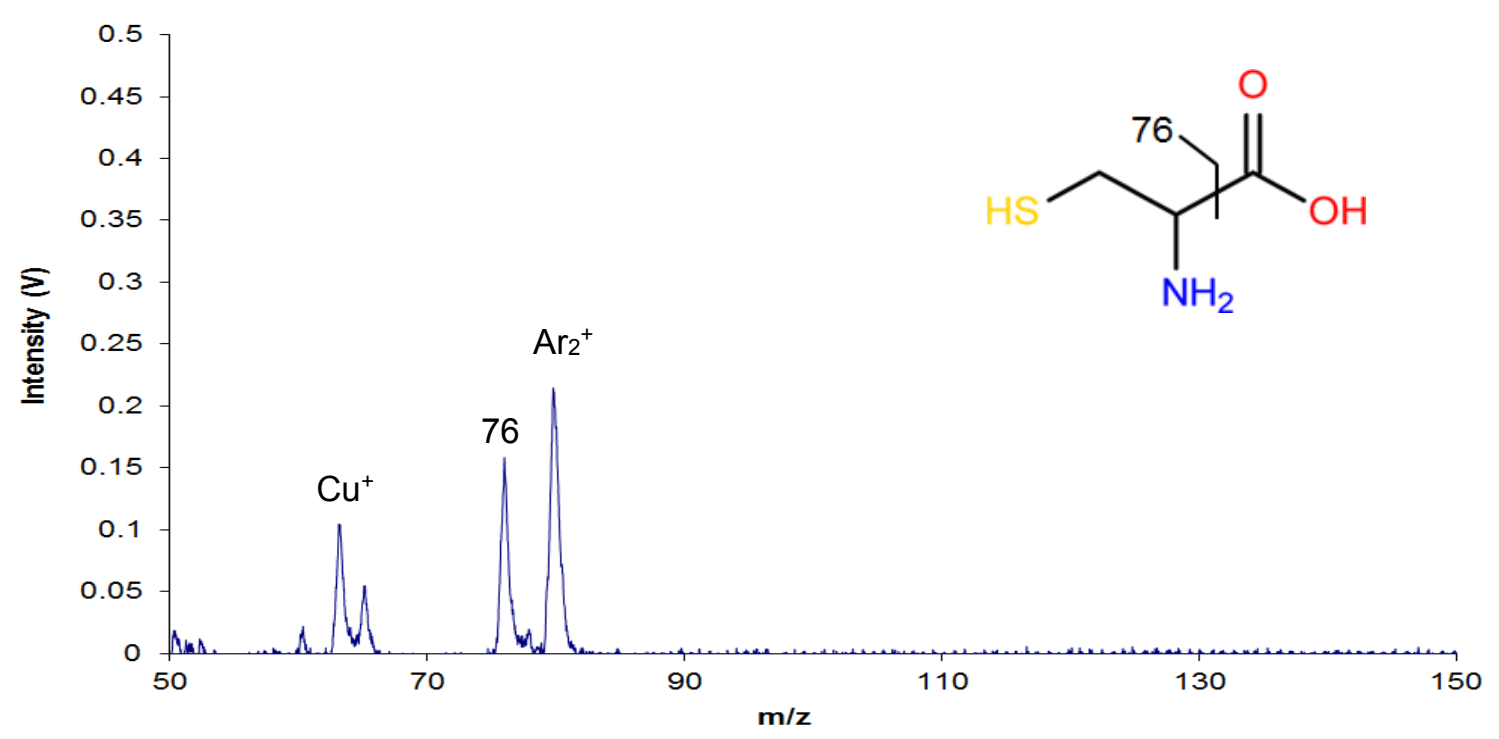

Figure 3.2 Mass spectra of (a) Blank, (b) $30 \mu \mathrm{g} / \mathrm{ml}$ cysteine during afterpeak (2.1 ms).

\subsubsection{Stability Tests of Characteristic Ion of Cysteine}

The cysteine solution residue film was like a thin layer of powder left in the reservoir after drying. These small quantities of cysteine residue would make the signal of cysteine on the surface decrease gradually during the sputtering process. Therefore, it is important to find the suitable sampling time to quantify the cysteine using this method. To achieve this goal, signal intensity monitoring tests for the selected ion signal was performed with different concentration of cysteine solutions. Fig. 3.3 shows the temporal intensity profiles 
of characteristic ion peak of cysteine at different concentrations. The peak intensity change mode as time goes on is found to be similar for samples of different concentrations. The peak intensity drops faster at first 3 mins and then reaches a quasisteady state between the fourth and fifth timing points for concentrations from $10 \mu \mathrm{g} / \mathrm{ml}$ to $40 \mu \mathrm{g} / \mathrm{ml}$. Therefore, the average intensities of ion peaks at $\mathrm{m} / \mathrm{z} 76$ for the fourth and fifth timing points were used to make the calibration curve.

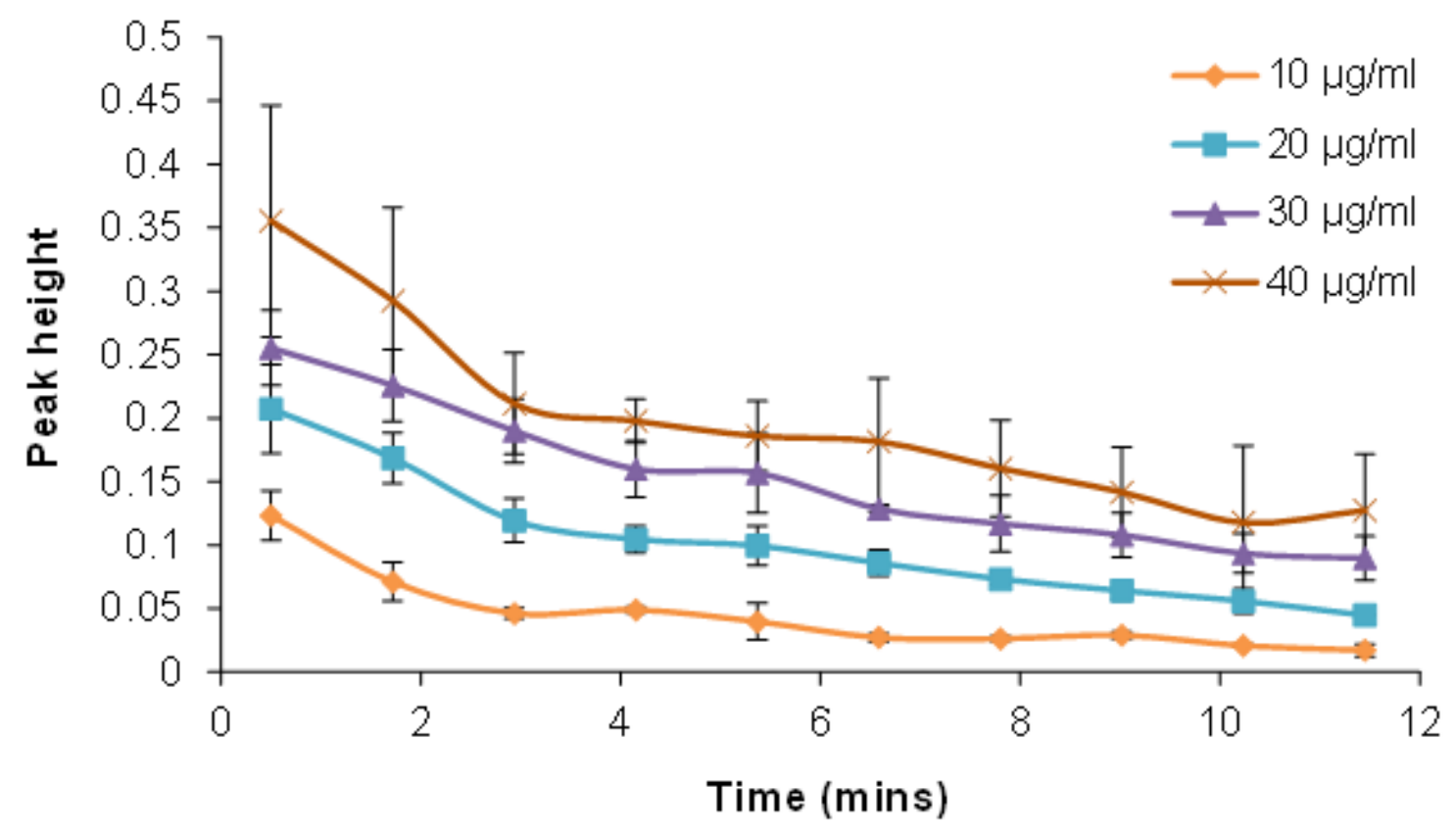

Figure 3.3 Temporal intensity profiles of characteristic ion peak $(\mathrm{m} / \mathrm{z} 76)$ of cysteine at different concentrations. 


\subsubsection{Quantitative Analysis of Cysteine}

The average quasi-steady intensities of 3 runs for each concentration were used for calibration. In Fig. 3.4, it shows the calibration curve of cysteine based on the peak height intensities of characteristic ion of cysteine $(\mathrm{m} / \mathrm{z} 76)$. The error bars on the points of calibration curve correspond to the standard deviations of 3 replicates. The error bar tends to increase with concentration. The Fig. 3.4a shows the calibration curve for cysteine standards with concentration ranges from $10 \mu \mathrm{g} / \mathrm{ml}$ to $40 \mu \mathrm{g} / \mathrm{ml}$. The correlation coefficient is 0.986 for this curve. The calibration curve including $0 \mu \mathrm{g} / \mathrm{ml}$ was also obtained, with a correlation coefficient of 0.9912 (Fig. 3.4b). Both of the calibration curves show good linearity. It proves the ability to quantify the cysteine by characteristic ion peak using GD-MS. 
(a)

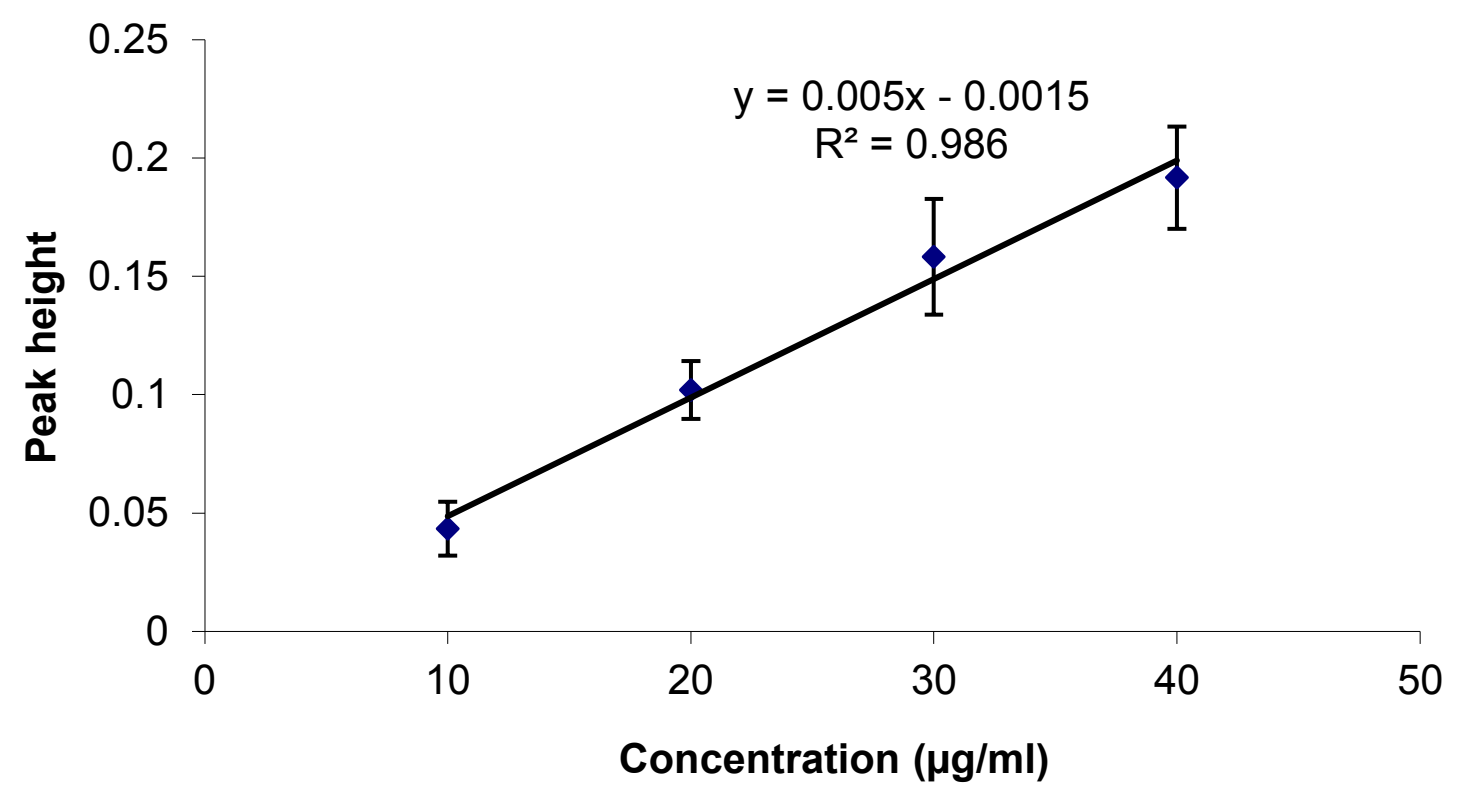

(b)

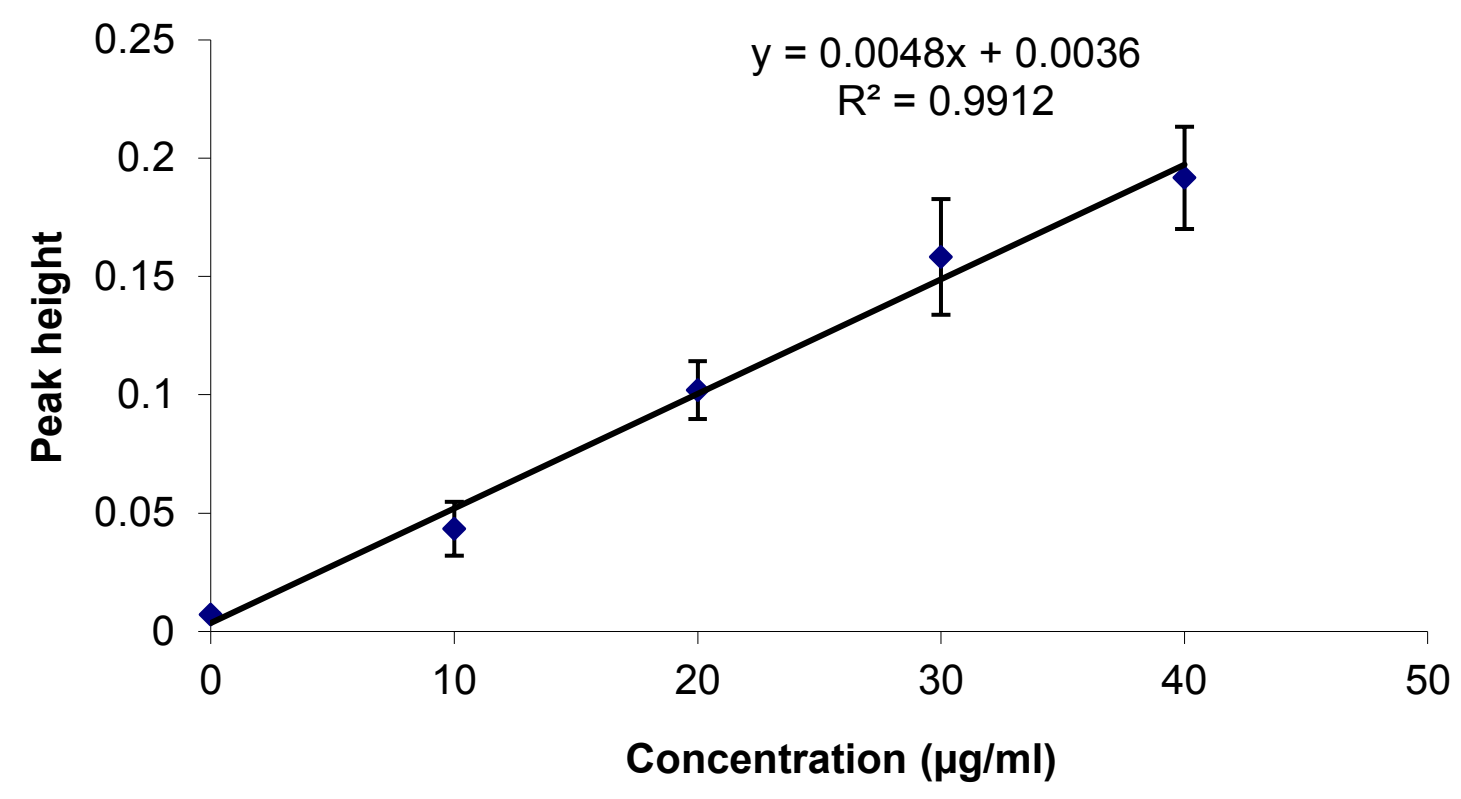

Figure 3.4 Calibration curves for cysteine standards based on the peak height intensity of $[\mathrm{M}-\mathrm{COOH}]^{+}(\mathrm{m} / \mathrm{z} 76)$ during afterpeak (2.1ms). 


\subsection{Conclusions}

In summary, the characteristic fragment ion at $\mathrm{m} / \mathrm{z} 76$ was found for analysis of cysteine. The calibration curve was generated using this fragment ion and show good linearity. With control of sample preparation procedure, the quantification of cysteine in solution was demonstrated using the time-of-flight pulsed glow discharge mass spectrometry. 


\subsection{References}

1. Bogaerts, A.; Gijbels, R., New Developments and Applications in GDMS, Fresenius J. Anal. Chem. 1999, 364, 367-375.

2. Marcus, R. K.; Broekaert, J. A. C., Glow discharge plasma in analytical spectroscopy 2003.

3. King, F. L.; Teng, J.; Steiner, R. E. Glow Discharge Mass Spectrometry: Trace Element Determinations in Solid Samples, J. Mass. Spectrom., 1995, 30, 10611075.

4. Li, L.; Barshick, C.M.; Millay, J. T.; Welty, A.V.; King, F. L., Determination of Bromine in Flame-Retardant Plastics Using Pulsed Glow Discharge Mass Spectrometry, Anal. Chem., 2003, 75, 3953-3961.

5. Duckworth, D.C.; Donohue, D. L.; Smith D.H., Design and Characterization of a Radio-Frequency-Powered Glow Discharge Source for Double-Focusing Mass Spectrometers, Anal. Chem. 1993, 65, 2478-2484.

6. McLuckey, S. A.; Glish, G. L.; Asano, K. G., Coupling of an Atmospheric Sampling Ion Source with an Ion Trap Mass Spectrometer, Anal. Chim. Acta. 1989, 225, $25-$ 35.

7. Fliegel, D.; Waddell, R.; Majidi, V.; Günther, D.; Lewis, C.L., Quantification of Aromatic and Halogenated Hydrocarbons and Alcohol Mixtures at the Elemental, Structural, and Parent molecular lon Level, Anal. Chem., 2005, 77, 1847-1852.

8. Montes Bayón, M.; B'Hymer, C.; Ponce de León , C. A.; Caruso, Joseph A., The Use of Radiofrequency Glow Discharge-Mass Spectrometry (rf-GDMS) Coupled 
to Gas Chromatography for the Determination of Selenoaminoacids in Biological Samples, J. Anal. At. Spectrom., 2001, 16, 492-497.

9. Daughtrey, E. H., Jr.; Harrison, W. W. Critical Parameters Affecting the Hollow Cathode lon Source, Anal. Chem., 1975, 47, 1024-1028.

10. Jakubowski, N.; Stuewer, D.; Toelg, G., Microchemical Determination of Platinum and Iridium by Glow Discharge Mass Spectrometry. Spectrochim. Acta Part B: Atomic Spectroscopy, 1991, 46B, 155-163.

11. Evetts, I.; Milton, D.; Mason, R., Trace Element Analysis in Body Fluids by Glow Discharge Mass Spectrometry: A Study of Lead Mobilization by the Drug cis-Platin, Biol. Mass Spectrom., 1991, 20, 153-159.

12. Mason, R.; Milton, D., Glow Discharge Mass Spectrometry of Some Organic Compounds, Int. J. Mass Spectrom. Ion Processes, 1989, 91, 209-225.

13. Gibeau, T. E.; Marcus, R. K., Glow Discharge Ionization Source for Liquid Chromatography/Particle Beam Mass Spectrometry. Anal.Chem., 2000, 72, 38333840.

14. Gibeau, T. E.; Marcus, R. K., Separation and Identification of Organic and Organometallic Compounds by Use of A Liquid Chromatography-Particle BeamGlow Discharge Mass Spectrometry Combination, J. Chromatogr. A, 2001, 915, $117-128$.

15. Venzie, J. L.; Davis, W. C.; Marcus, R. K., Organic and Inorganic Arsenic Speciation Through Ion Exchange Chromatography with Particle Beam-Glow Discharge Mass Spectrometry Detection, J. Anal. At. Spectrom., 2004, 19, 13091314. 
16. Brewer, T. M.; Castro, J.; Marcus, R. K., Particle Beam Sample Introduction into Glow Discharge Plasmas for Speciation Analysis. Spectrochim. Acta, Part B: Atomic Spectroscopy, 2006, 61B, 134-149.

17. Matsumoto, K.; Kojima, H.; Yasuda, K.; Tsuge, S., Chemical Ionization Mass Spectrometry Using a Glow Discharge Ion Source Combined With a Nebulizer Sampling System, Org.Mass Spectrom., 1985, 20, 243-246.

18. Zhao, J.; Zhu, J.; Lubman, D. M., Liquid Sample Injection Using an Atmospheric Pressure Direct Current Glow Discharge Ionization Source, Anal .Chem., 1992, 64, $1426-1433$.

19. Rampler, E.; Dalik, T.; Stingeder, G.; Hann, S.; Koellensperger, G., Sulfur Containing Amino Acids - Challenge of Accurate Quantification, J. Anal. At. Spectrom., 2012, 27, 1018-1023.

20. Rappel, C.; Schaumlöeffel, D., The Role of Sulfur and Sulfur Isotope Dilution Analysis in Quantitative Protein Analysis, Anal. Bioanal. Chem., 2008, 390, 605615.

21. Harper, A. E.; Benevenga, N. J.; Wohlhueter, R. M., Effects of Ingestion of Disproportionate Amounts of Amino Acids, Physiol. Rev., 1970, 50, 428-558.

22. Masella, Roberta; Mazza, Giuseppe, Glutathione and Sulfur Amino Acids in Human Health and Disease, 2009, 291-316.

23. Benninghoven, A.; Jaspers, D.; Sichtermann, W., Secondary Ion Emission of Organic Compounds: Amino Acids, Adv. Mass Spectrom., 1978, 7B, 1433-1436.

24. Steiner, R. E.; Lewis, C. L.; King, F. L., Time-of-Flight Mass Spectrometry with a Pulsed Glow Discharge Ionization Source, Anal. Chem. 1997, 69, 1715-1721. 
25. Bruhn C. G.; Harrison, W. W., Sputter-Atomization Studies with a Glow Discharge, Anal. Chem. 1978, 50, 16-21.

26. http://webbook.nist.gov/cgi/cbook.cgi?Name=cysteine\&Units=SI\&cMS=on

27. Zhang, N.; King, F. L., Direct Manganese (Mn) Speciation in Solid State Materials by Pulsed Glow Discharge Time-of-Flight Mass Spectrometry, J. Anal. At. Spectrom., 2009, 24, 1489-1497.

28. Robertson-Honecker, J. N.; Zhang, N.; Pavkovich, Alexandria; King, F. L., Direct Chromium Speciation in Solid State Materials-a GDMS Approach, J. Anal. At. Spectrom., 2008, 23, 1508-1517.

29. Gu, G.; DeJesus, M.; King, F. L., Direct FexOy Speciation in Solid State Materials by Pulsed Millisecond Radio Frequency Glow Discharge Time-of-Flight Mass Spectrometry, J. Anal. At. Spectrom., 2011, 26, 816-821.

30. Li, L.; Millay, J. T.; Turner, J. P.; King, F. L., Millisecond Pulsed Radio Frequency Glow Discharge Time of Flight Mass Spectrometry: Temporal and Spatial Variations in Molecular Energetics, J. Am. Soc. Mass Spectrom., 2004, 15, 87102.

31. Pan, C.; King, F. L., Ion Formation Processes in the Afterpeak Time Regime of Pulsed Glow Discharge Plasmas, Journal of the American Society for Mass Spectrometry, 1993, 4, 727-732. 


\title{
Chapter 4
}

\section{Direct $\mathrm{Nb}_{\mathrm{x}} \mathrm{O}_{\mathrm{y}}$ Speciation in Solid State Materials by Pulsed Glow Discharge Time-of-Flight Mass Spectrometry}

\author{
Abstract
}

Glow discharge mass spectrometry (GDMS) is a well suitable technique for direct analysis of trace elements in solid state sample. Previous work developed a method to use GDMS for direct speciation of chromium (III, VI) and manganese (II, IV) in solid state samples based on specific cluster ions. Differentiation of three iron oxides has also been demonstrated by comparing ion abundance ratios using GDMS. In present work, the direct speciation of $\mathrm{Nb}_{\mathrm{x}} \mathrm{O}_{\mathrm{y}}$ in solid state materials is an extended application of GDMS. The effects of operating parameters, such as sampling time, sampling distance and power, to ion signal profiles are studied. It is found that the stoichiometry of metal oxides show a strong correlation with relative abundance distribution of metal oxide clusters ions and atomic ions, which is similar to the observation in iron oxide speciation using GDMS. With different signal intensity ratio between niobium oxide cluster ions and niobium atomic ion, the differentiation between $\mathrm{NbO}, \mathrm{NbO}_{2}$, and $\mathrm{Nb}_{2} \mathrm{O}_{5}$ is achieved under optimal operating conditions of pulsed glow discharge time-of-flight mass spectrometry. 


\subsection{Introduction}

Chemical speciation is of increased interest in many fields, such as environmental, nutritional, and materials chemistry. ${ }^{1-3}$ Elemental oxidation state speciation, one kind of chemical speciation, is very important because it can affect toxicity, bioavailability and transport of the element or affect properties of a material. ${ }^{3-6}$ For elemental speciation of a solid state sample, traditional methods usually involves separation methods (i.e. liquid chromatography and gas chromatography) coupled with elemental detection techniques (i.e. atomic spectroscopy and electrochemical detection). In general, the pretreatment procedures of the solid sample are required before analysis. For example, solid sample often need to be dissolved with solvents and may need other procedures like extraction, preconcentration or derivatization. These pretreatment procedures not only make the analysis time consuming, but also may change the elemental oxidation state in original sample. $^{4-8}$

To avoid alteration of elemental oxidation state in original sample during analysis, direct elemental speciation in solid state is preferred. X-ray techniques are widely used for direct elemental speciation in solid state materials. ${ }^{4}$ For example, the identification of the oxidation state of niobium at the metal/oxide interface of $(\mathrm{Zr}, \mathrm{Nb})$ alloys was performed using X-ray absorption near-edge structure (XANES) spectroscopy. ${ }^{1}$ In addition, several mass spectrometry based techniques with different ionization sources, such as laser microprobe mass spectrometry (LMMS), laser ablation mass spectrometry (LA-MS), and static secondary ion mass spectrometry (s-SIMS), are used for speciation of elemental 
oxidation state. ${ }^{7}$ The previous studies found that the stoichiometry and valence of metal oxide show a strong correlation with relative abundance distribution of clusters ions and atomic ions. ${ }^{9-11}$ Michiels et al. used ion abundance ratios to differentiate three titanium oxides by LMMS. ${ }^{10}$ The iron oxides speciation was also demonstrated using the similar method via LMMS with time-of-flight analyzer. ${ }^{9}$ Aubriet et al. discriminated first-row transition metal oxides based on three methods, which are characteristic ions, ion abundance ratios, and a valence model. ${ }^{7}$

Glow discharge mass spectrometry (GDMS) is a well-established technique for direct analysis of trace elements in solid state sample. It separates the sputtering and ionization processes in time and space, leading to a decreased dependence of analytical signal on matrix and a representative population of species from the original sample. ${ }^{12}$ Introduction of pulsed glow discharge makes the mass spectra tunable in time because it provides different ionization environments in three different time regimes: prepeak, plateau, and afterpeak. ${ }^{13}$ Variations in temporal and spatial sampling as well as variations in operating power enable tuning of the internal energy distribution of the glow discharge so that the chemical speciation can be performed at elemental, structural and molecular level. ${ }^{14}$ Using GDMS coupled with gas chromatography (GC), Lewis et al. demonstrated that elemental, structural and molecular information can be obtained nearly simultaneously. ${ }^{15}$ Later on, the application of GDMS to chemical speciation was extended to speciation of elemental oxidation state in this lab. With pulsed glow discharge time-offlight mass spectrometry, direct speciation of chromium (III, VI) and manganese (II, IV) in solid state samples were achieved based on characteristic ions. ${ }^{5-6}$ Gu et al. successfully differentiated $\mathrm{FeO}, \mathrm{Fe}_{2} \mathrm{O}_{3}$, and $\mathrm{Fe}_{3} \mathrm{O}_{4}$ by comparing ion abundance ratios using the same 
technique. ${ }^{16}$ All these three metals, studied for speciation of their oxides using GDMS, belong to the first row of transition metals.

The present work extends the application of pulsed glow discharge time-of-flight mass spectrometry to the direct speciation of niobium, which is a transition metal in second row. Niobium and its oxides are promising as a material for superconducting materials, semiconductors, specialty glasses, catalysts and sensors. ${ }^{1}, 3,17-21$ Niobium oxidation state speciation is important because the performance of a material can be affected by oxidation state of niobium. ${ }^{1,3,17,19}$ In this work, we adapted the techniques developed for elemental speciation of other elements in this lab to discriminate between $\mathrm{NbO}, \mathrm{NbO}_{2}$, and $\mathrm{Nb}_{2} \mathrm{O}_{5}$. Temporal ion signal profiles of niobium oxides $\left(\mathrm{Nb}_{x} \mathrm{O}_{y}\right)$ are presented, and the effect of operating conditions (i.e. sampling distance and power) on these ion signals are discussed. Under optimized conditions, the differentiation of three niobium oxides is achieved based on ion abundance ratios. This work further demonstrates the ability of pulsed GDMS to provided elemental oxidation state speciation by ion abundance ratios.

\subsection{Experimental}

\subsubsection{Sample Preparation}

Analytical reagent grades of niobium (II) oxide (NbO), niobium (IV) oxide $\left(\mathrm{NbO}_{2}\right)$, niobium $(\mathrm{V})$ oxide $\left(\mathrm{Nb}_{2} \mathrm{O}_{5}\right)$ were purchased from commercial suppliers (Alfa Aesar, Ward Hill, MA). Three niobium oxides were mixed with spectroscopic grade copper powder 
(Matheson Company Inc., East Rutherlord, NJ) separately at 1:3 (w:w) ratio. The mixed powders were then homogenized for 2 minutes using a laboratory mixer (Wig-L-Bug, SPEX, Edison, NJ). Each mixture was then compacted at 4000 psi for 30 min to produce sample disks with $5 \mathrm{~mm}$ diameter and $3.5 \mathrm{~mm}$ height. All constituent powders and pressed pellets were baked overnight in oven at $110^{\circ} \mathrm{C}$ before use.

\subsubsection{Pulsed Glow Discharge TOF Mass Spectrometer}

The GD plasma was supplied by powering a $13.56 \mathrm{MHz}$ radio frequency (RF) generator (Model RF 10-S, RF Plasma Product Inc., Marlton, NJ) coupled with an automatic matching network (Model AM-10, RF Plasma Product Inc., Marlton, NJ). The applied power, pulse width, and duty cycle were controlled by the RF power supply system in internal pulsing mode. In this work, the pulse width was set at $5 \mathrm{~ms}$ with a duty cycle of $25 \%$. Ultra pure argon (Airgas, Randor, PA), used as glow discharge supporting gas, was introduced into the glow discharge chamber. A metering valve was used to control the gas pressure at 0.3 Torr, which was monitored by a thermocouple pressure gauge (Varian, Lexington, MA).

Sample disks were introduced onto the tip of the direct insertion probe (DIP) and used as the glow discharge cathode. The sample cathodes were surrounded by a MACOR shield (Accuratus Corporation Phillipsburg, $\mathrm{NJ}$ ) to make the sputtering occur only at the top surface of the sample disk. Finally, the DIP was inserted into the glow discharge chamber for 20 minutes before turning on the power. The mass spectrometer used in this study is a linear time-of-flight (TOF) mass spectrometer (R.M. Jordan Co., Grass Valley, 
$\mathrm{CA}$ ), which is positioned orthogonally to the ion beam from glow discharge. Operation conditions for the pulsed glow discharge time-of-flight mass spectrometer are shown in Table 4.1. Further details of this mass spectrometer and data collection were described previously. ${ }^{5-6,22}$

Table 4.1 Pulsed RF GD TOF-MS operating parameters

\section{Glow discharge}

Pressure

$$
\begin{gathered}
0.3 \text { Torr } \\
90-130 \mathrm{~W} \\
5 \mathrm{~ms}
\end{gathered}
$$

Operating power

Pulse duration

Duty cycle $25 \%$

Sampling distance

$7-15 \mathrm{~mm}$

\section{Time of Flight}

Flight path length

$1 \mathrm{~m}$

Ion lenses potential

Skimmer

Extractor

Repeller

$+250 \mathrm{~V}$

Accelerator

$-1535 \mathrm{~V}$

Deflector (X1)

$-1900 \mathrm{~V}$

Deflector $(\mathrm{Y} 1)$

$-1550 \mathrm{~V}$

Detector

$$
-1895 \mathrm{~V}
$$

Vacuum conditions

Intermediate stage

$10^{-5}$ Torr

Flight tube

$10^{-6}$ Torr 


\subsection{Results and Discussion}

\subsubsection{Evaluation of Temporal Regimes}

The application of pulsed glow discharge creates three distinct temporal regimes: prepeak, plateau, and afterpeak regimes. The different ionization environment over the pulse cycle results in variation of ion signal profiles for pure copper and niobium species. ${ }^{14}$ To compare the mass spectra of copper and niobium species in three temporal regimes, the glow discharge was operated using 0.3 Torr argon, 110W RF power, and $5 \mathrm{~ms}$ with $25 \%$ duty cycles. The sampling distance from cathode surface was $10 \mathrm{~mm}$. Three types of niobium oxides $\left(\mathrm{Nb}_{x} \mathrm{O}_{y}\right)$ were investigated in this experiment: $\mathrm{NbO}, \mathrm{NbO}_{2}$, and $\mathrm{Nb}_{2} \mathrm{O}_{5}$.

Prepeak. Once the power is applied, the induced electrical discharge results in the surge of energetic electrons. Consequently, the electron ionization is dominant during several hundred microseconds after ignition of the discharge plasma, known as prepeak regime. ${ }^{13.23}$ Figure 4.1 shows the representative prepeak regime mass spectra for pure copper and $\mathrm{Nb}_{x} \mathrm{O}_{y} / \mathrm{Cu}$ mixtures collected at $0.3 \mathrm{~ms}$ after power-on. For both pure copper and $\mathrm{Nb}_{x} \mathrm{O}_{y} / \mathrm{Cu}$ mixtures, discharge gas ions (i.e. $\mathrm{Ar}^{+}$and $\mathrm{Ar}^{2+}$ at $\mathrm{m} / \mathrm{z} 40$ and 20, respectively), arising from electron ionization between argon and energetic electrons, are the primary signals observed in the mass spectra. Ion signals from copper and niobium oxides are not observed in the prepeak because it takes some time for cathode species to be sputtered, diffuse into the negative glow and be ionized. The mass spectra of $\mathrm{NbO}$, $\mathrm{NbO}_{2}$ and $\mathrm{Nb}_{2} \mathrm{O}_{5}$ are similar to each other in the prepeak regime. 
(a) Pure copper

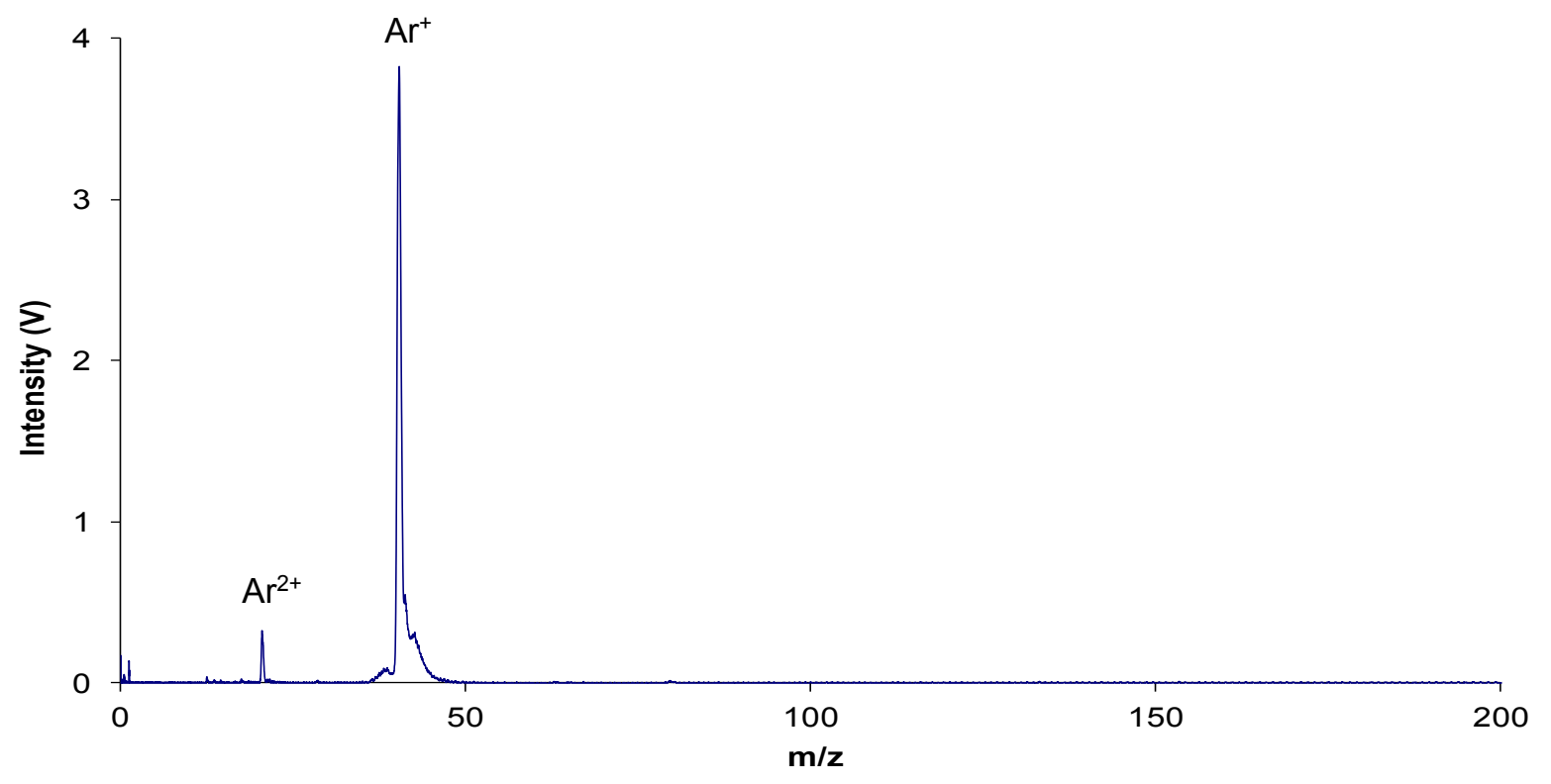

(b) $25 \% \mathrm{NbO}+75 \% \mathrm{Cu}$

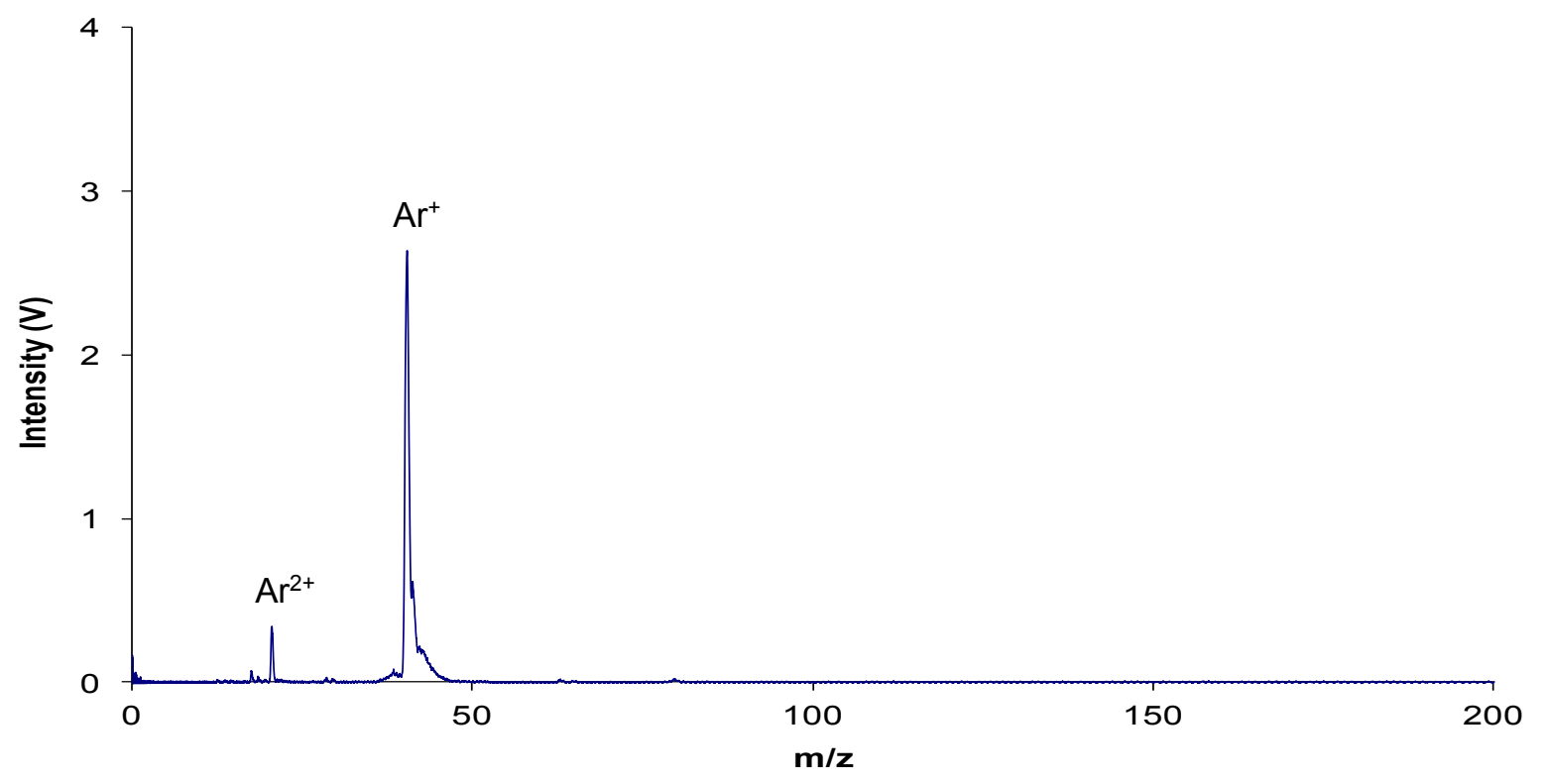


(c) $25 \% \mathrm{NbO}_{2}+75 \% \mathrm{Cu}$

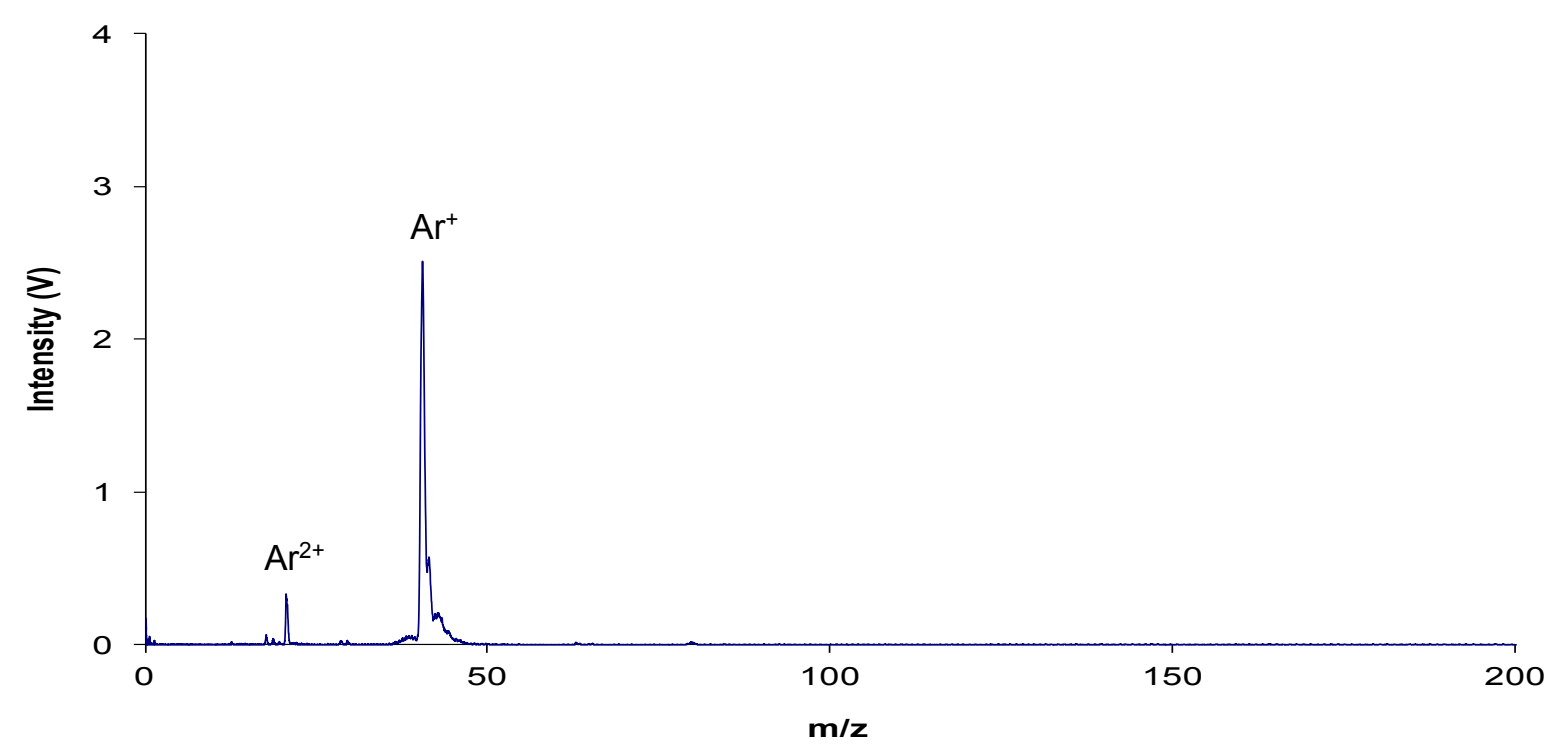

(d) $25 \% \mathrm{Nb}_{2} \mathrm{O}_{5}+75 \% \mathrm{Cu}$

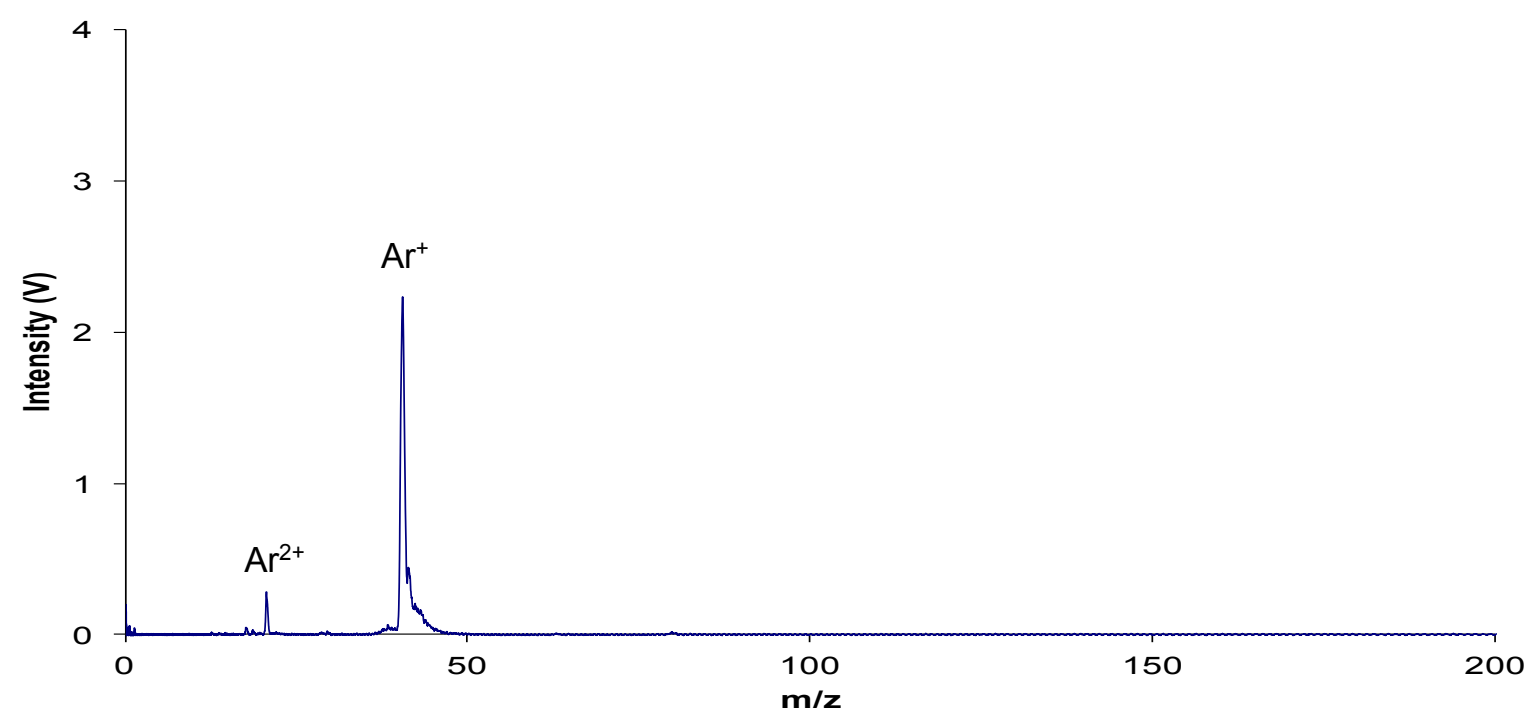

Figure 4.1 Time resolved mass spectra of (a) pure copper, (b) $25 \% \mathrm{NbO}+75 \% \mathrm{Cu}$, (c) $25 \% \mathrm{NbO}_{2}+75 \% \mathrm{Cu}$, and (d) $25 \% \mathrm{Nb}_{2} \mathrm{O}_{5}+75 \% \mathrm{Cu}$ samples during the plateau (0.3 ms). Operating conditions: discharge pressure 0.3 Torr, operating power $110 \mathrm{~W}$, pulse duration $5 \mathrm{~ms}$ with $25 \%$ duty cycle, and sampling distance $10 \mathrm{~mm}$. 
Plateau. At $\sim 1 \mathrm{~ms}$ after power pulse application, the glow discharge plasma reaches and remains at the quasi-equilibrium state until the power termination, this time domain is the plateau regime. During this period, the ionization process is a combination of electron ionization, Penning ionization and charge transfer ionization, which is analogous to what occurs in a continuous glow discharge. The ion signals of discharge gas and cathode material co-exist at the steady state in plateau region. ${ }^{5,13}$ As shown in Figure 4.2, plateau mass spectra of $\mathrm{Nb}_{x} \mathrm{O}_{y} / \mathrm{Cu}$ collected at $4.9 \mathrm{~ms}$ exhibit ion signals from argon gas, copper, and niobium oxides. For the mass spectra obtained from both pure copper and $\mathrm{Nb}_{x} \mathrm{O}_{y} / \mathrm{Cu}$ mixtures, the ion signals corresponding to argon gas are observed at $\mathrm{m} / \mathrm{z} 20\left(\mathrm{Ar}^{2+}\right), \mathrm{m} / \mathrm{z} 40\left(\mathrm{Ar}^{+}\right)$and $\mathrm{m} / \mathrm{z} 80\left(\mathrm{Ar}_{2}{ }^{+}\right)$, and the copper isotope peaks at $\mathrm{m} / \mathrm{z} 63$ and $\mathrm{m} / \mathrm{z} 65$ are also visible in the mass spectra. In addition, the ion signals from air are also present in the mass spectra, such as $\mathrm{H}_{2} \mathrm{O}^{+}(\mathrm{m} / \mathrm{z} 18)$ and fragment ion $\mathrm{OH}^{+}(\mathrm{m} / \mathrm{z} 17)$. In addition to these ion signals, the three types of niobium oxides exhibit both atomic ion $\left(\mathrm{Nb}^{+}\right.$at $\mathrm{m} / \mathrm{z}$ 93) and cluster ions $\left(\mathrm{NbO}^{+}\right.$at $\mathrm{m} / \mathrm{z} 109$ along with weaker signal $\mathrm{NbO}_{2}{ }^{+}$at $\mathrm{m} / \mathrm{z}$ $125)$ in the mass spectra. However, no specific ion was detected for $\mathrm{Nb}_{\mathrm{x}} \mathrm{O}_{\mathrm{y}} / \mathrm{Cu}$ to enable speciation in the plateau regime. 
(a) Pure copper

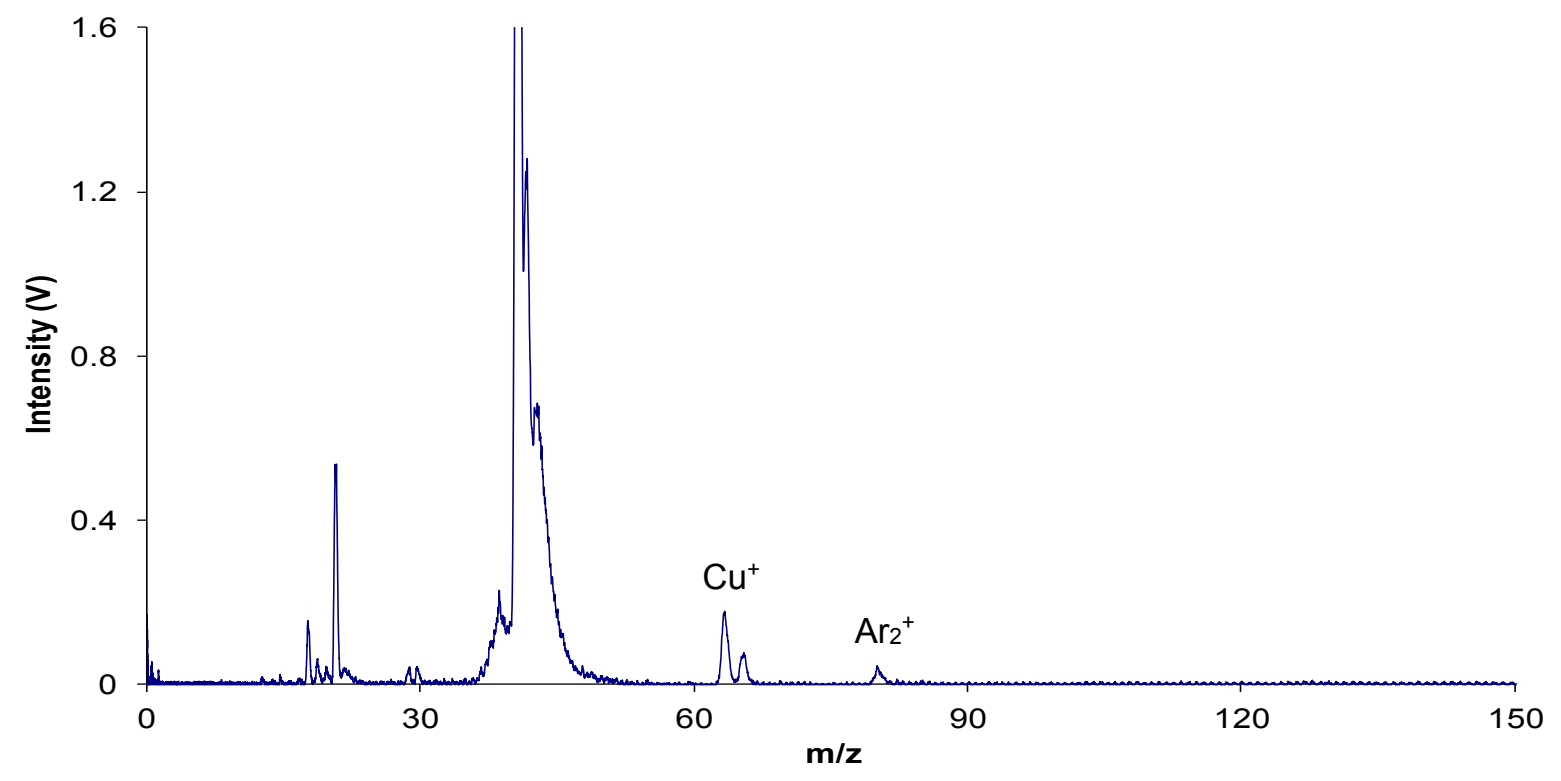

(b) $25 \% \mathrm{NbO}+75 \% \mathrm{Cu}$

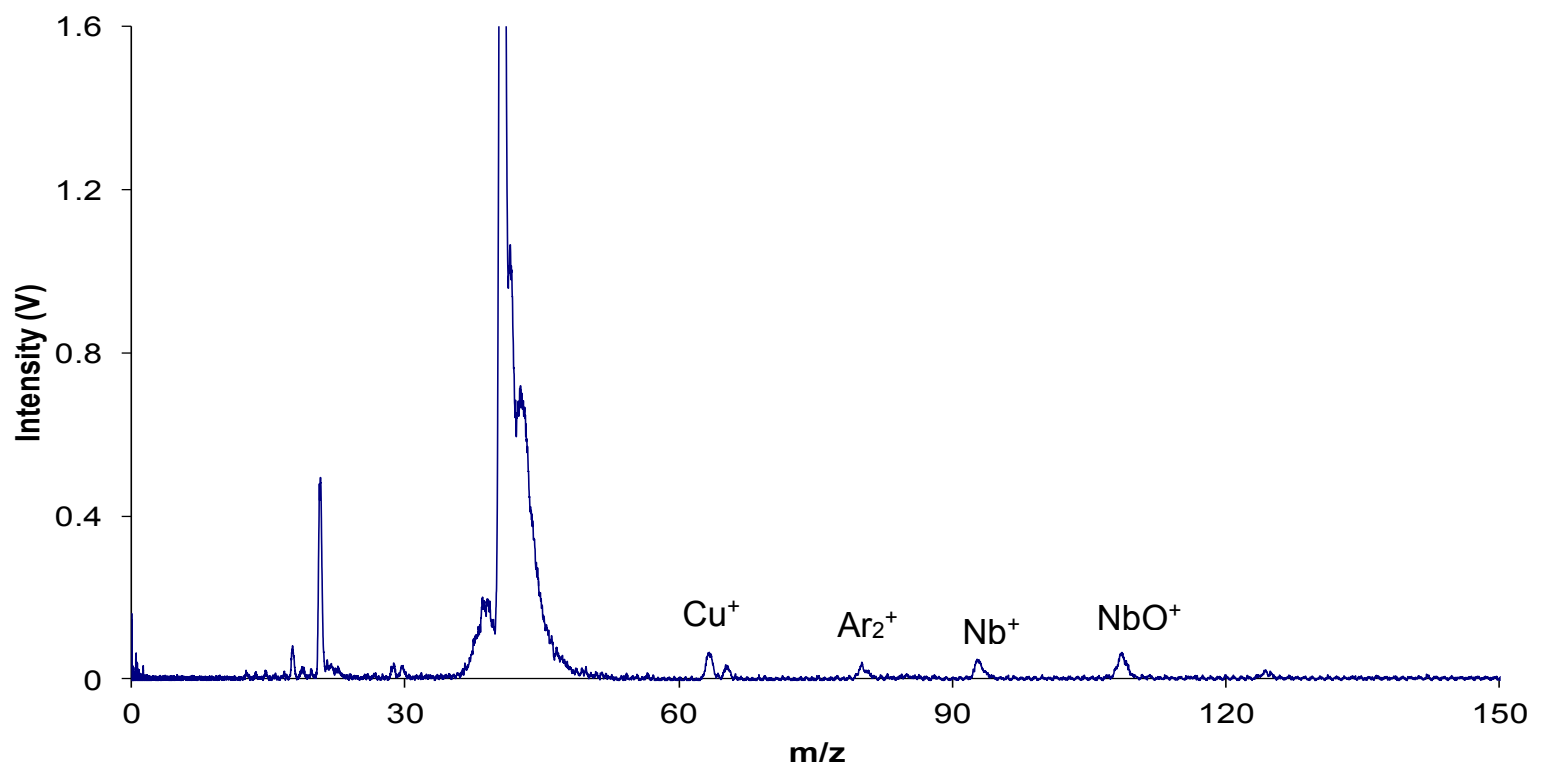


(c) $25 \% \mathrm{NbO}_{2}+75 \% \mathrm{Cu}$

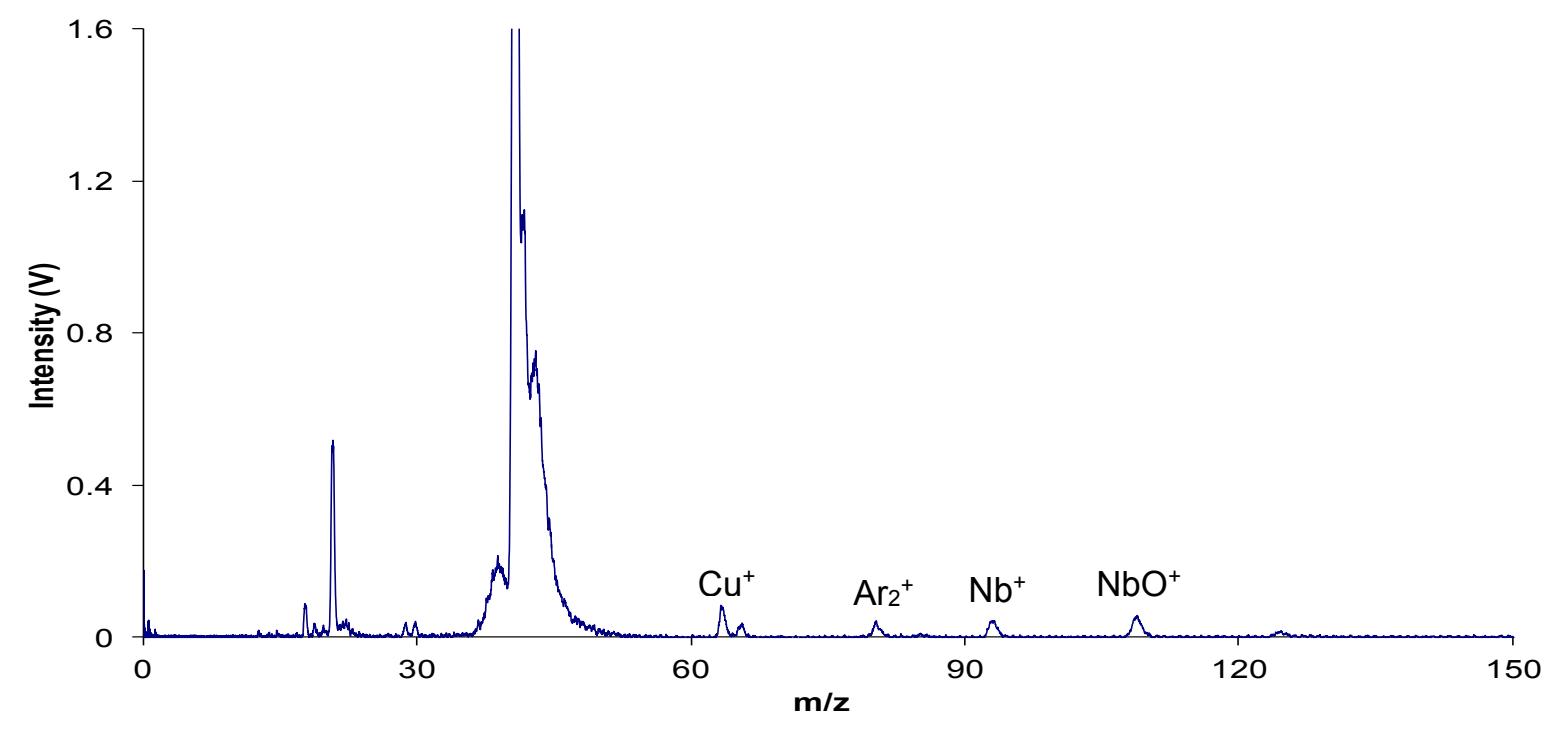

(d) $25 \% \mathrm{Nb}_{2} \mathrm{O}_{5}+75 \% \mathrm{Cu}$

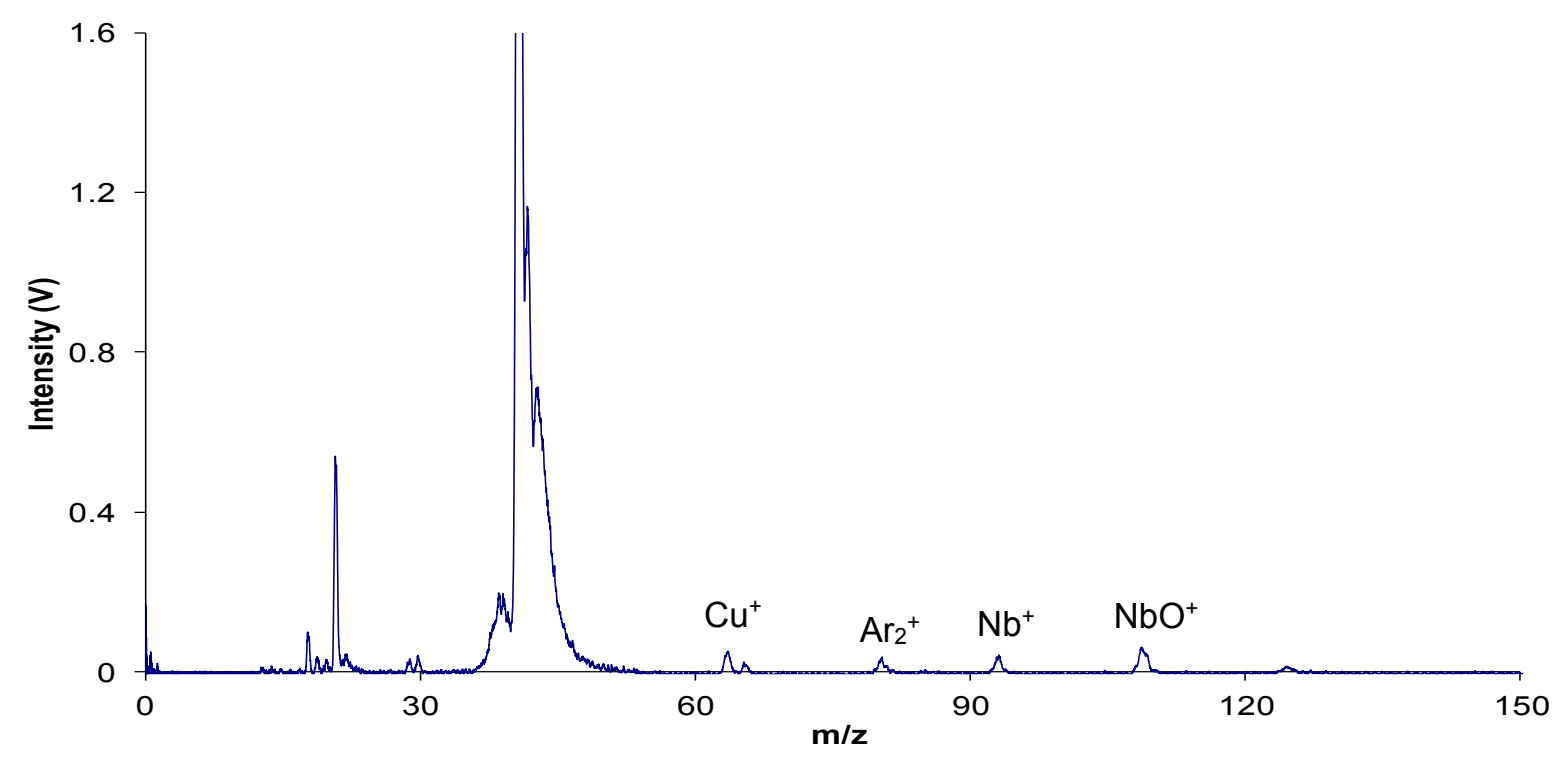

Figure 4.2 Time resolved mass spectra of (a) pure copper, (b) $25 \% \mathrm{NbO}+75 \% \mathrm{Cu}$, (c) $25 \% \mathrm{NbO}_{2}+75 \% \mathrm{Cu}$, and (d) $25 \% \mathrm{Nb}_{2} \mathrm{O}_{5}+75 \% \mathrm{Cu}$ samples during the plateau (4.9 ms). Operating conditions: discharge pressure 0.3 Torr, operating power $110 \mathrm{~W}$, pulse duration $5 \mathrm{~ms}$ with $25 \%$ duty cycle, and sampling distance $10 \mathrm{~mm}$. 
Afterpeak. Upon pulse power termination, the energetic electrons lose their kinetic energy through collision process. The recombination of thermalized electrons and argon ions results in the surge of metastable argon atoms which enhances the Penning ionization in afterpeak regime. Also, cathode species signals are enhanced by the increased population of metastable argon atoms. Previous studies found that Penning ionization dominates in the afterpeak regime. ${ }^{13,24-25}$ Compared to electron ionization and charge transfer ionization, Penning ionization prefers to generate larger ions like molecular ions or cluster ions because it affords relatively soft ionization. ${ }^{14}$ Traditionally, this region is more useful for metal oxide speciation. ${ }^{5-6,16}$

(a) Pure copper

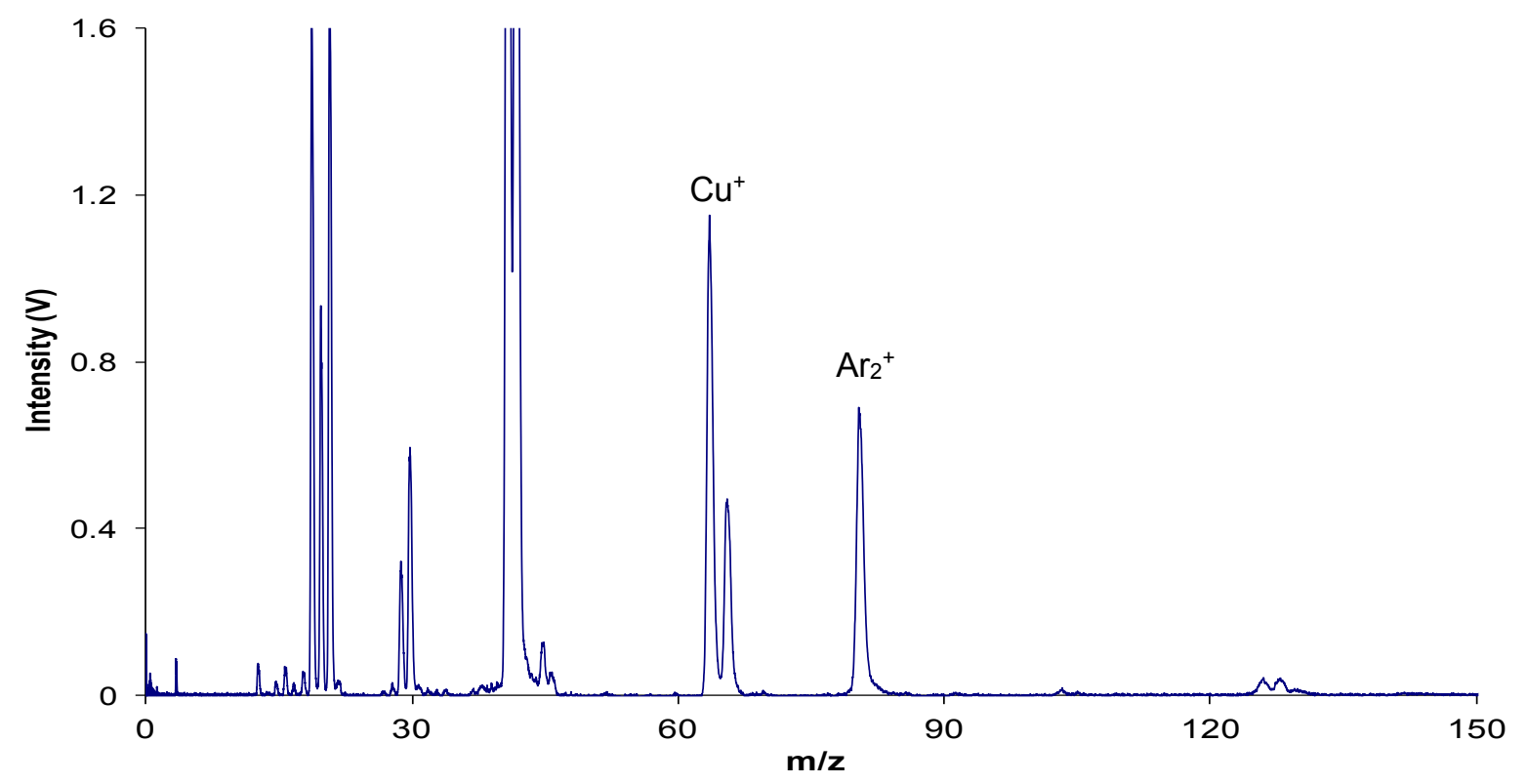


(b) $25 \% \mathrm{NbO}+75 \% \mathrm{Cu}$

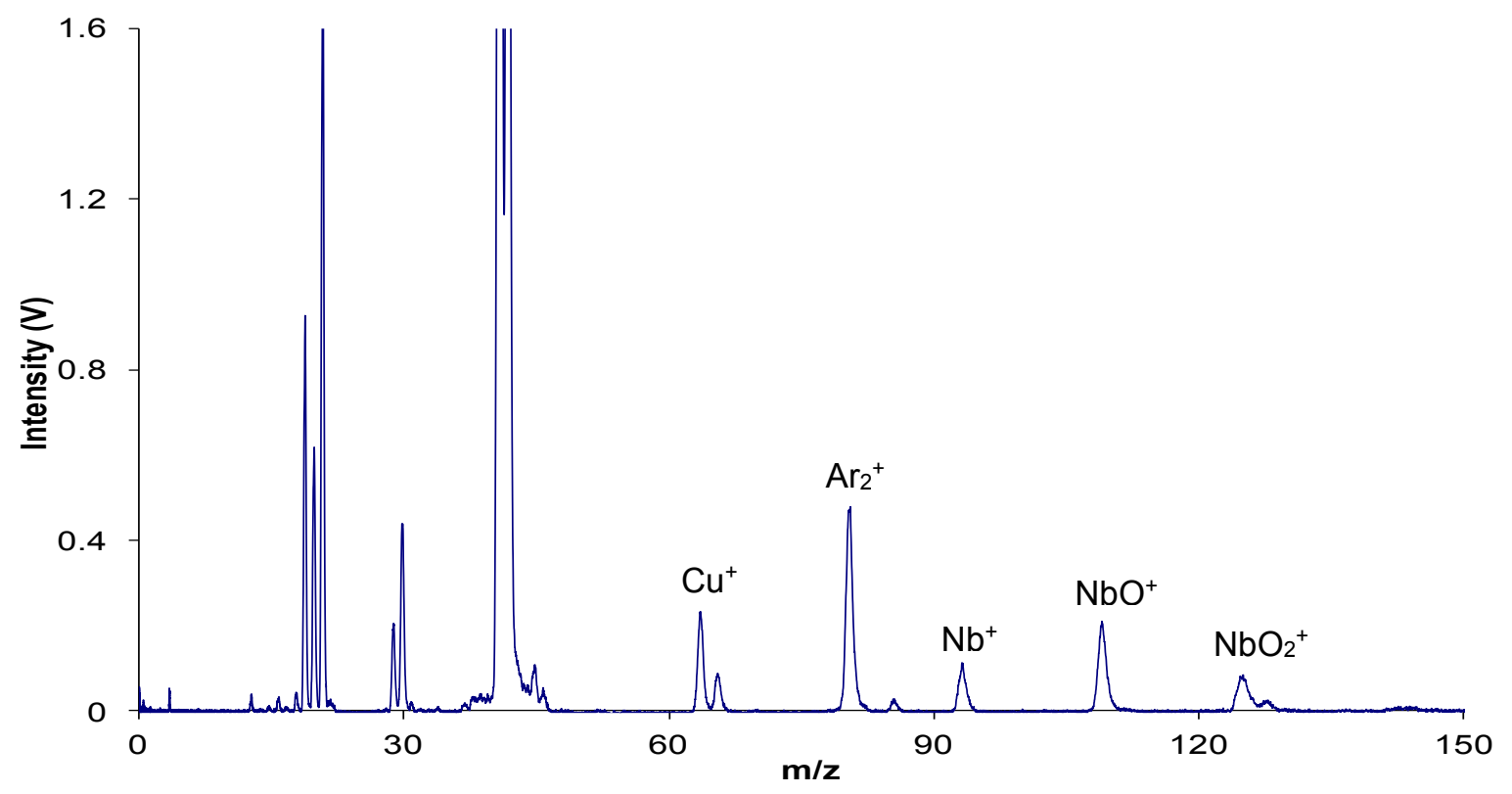

(c) $25 \% \mathrm{NbO}_{2}+75 \% \mathrm{Cu}$

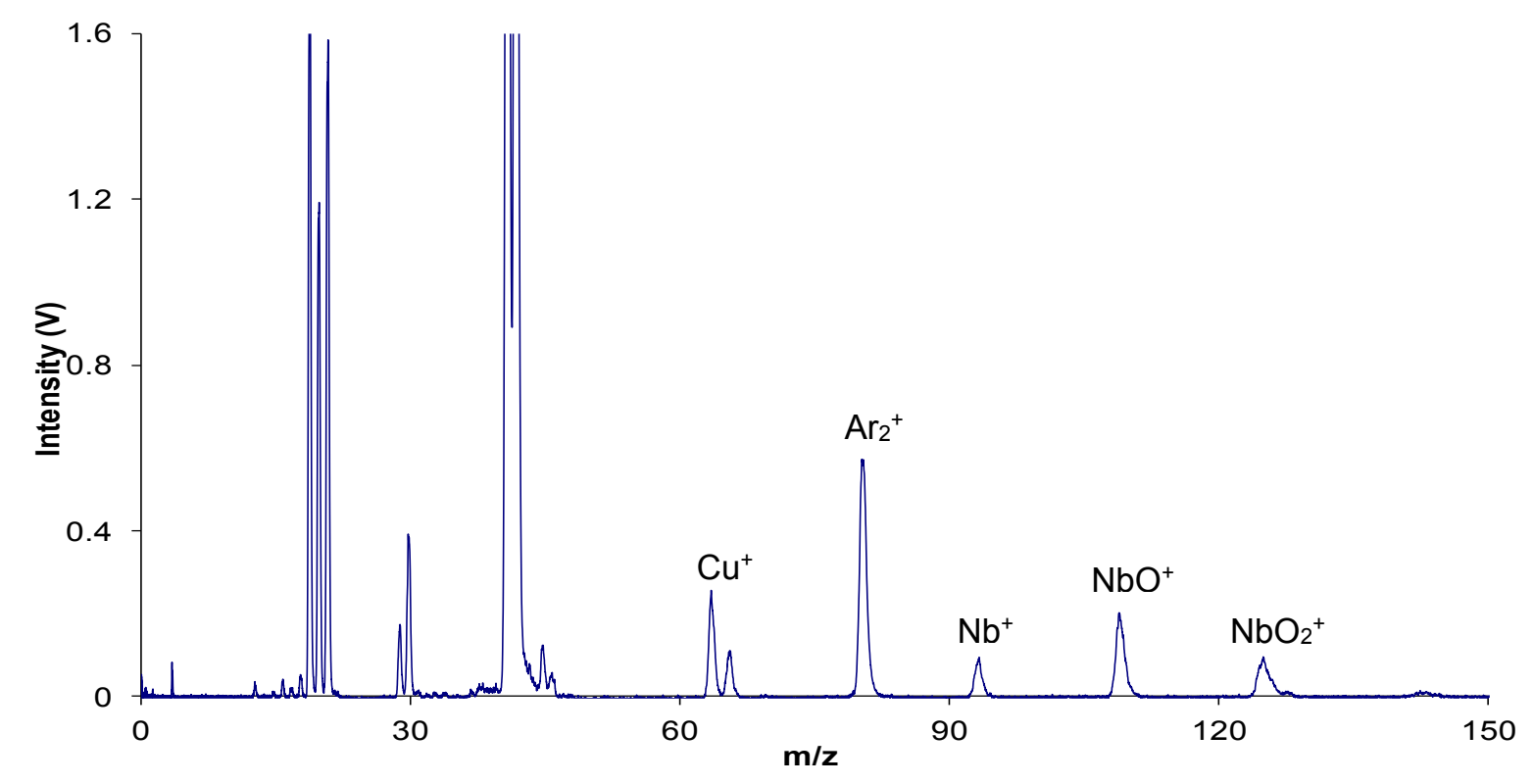


(d) $25 \% \mathrm{Nb}_{2} \mathrm{O}_{5}+75 \% \mathrm{Cu}$

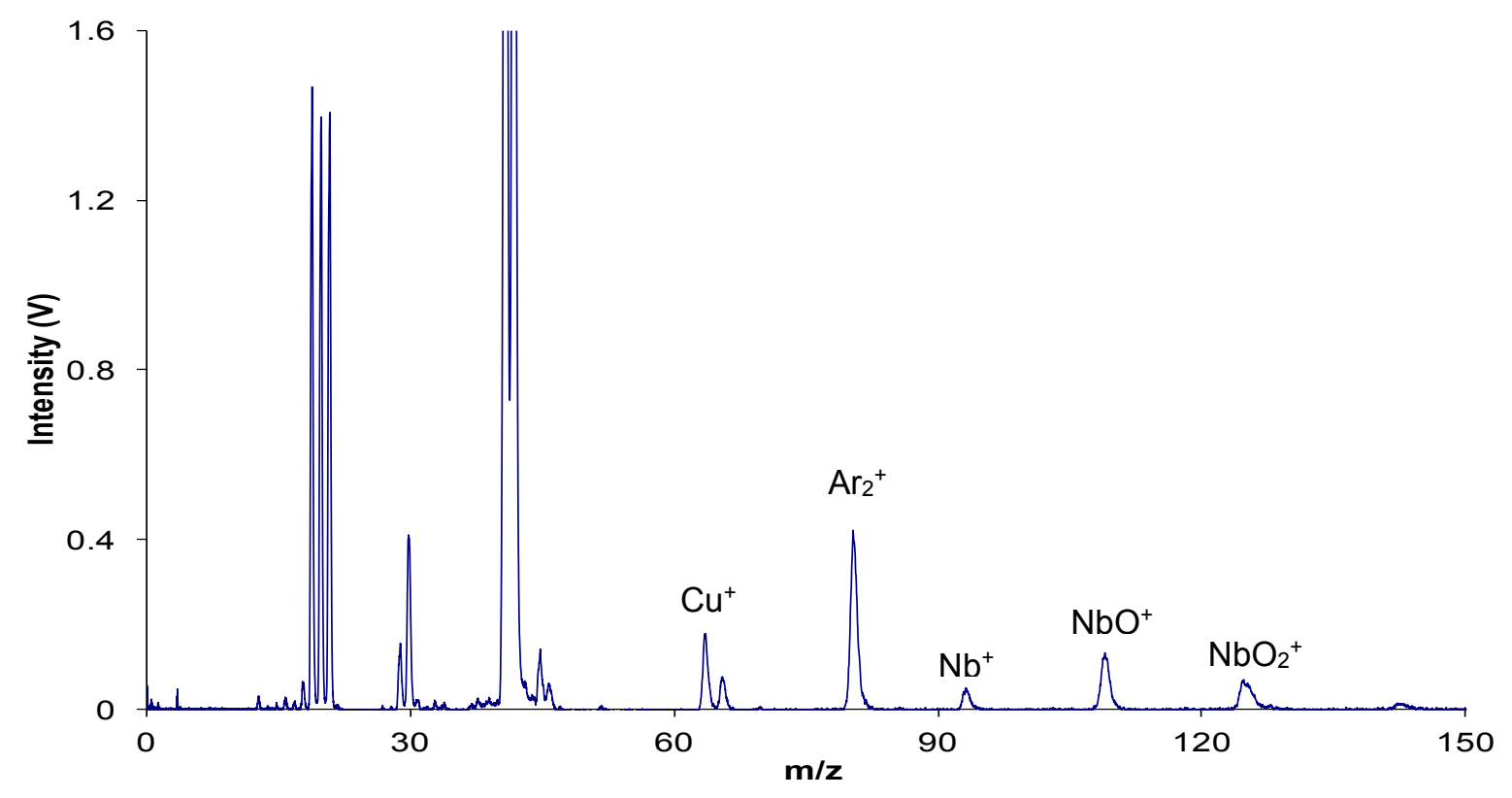

Figure 4.3 Time resolved mass spectra of (a) pure copper, (b) $25 \% \mathrm{NbO}+75 \% \mathrm{Cu}$, (c) $25 \% \mathrm{NbO}_{2}+75 \% \mathrm{Cu}$, and (d) $25 \% \mathrm{Nb}_{2} \mathrm{O}_{5}+75 \% \mathrm{Cu}$ samples during the plateau (5.2 ms). Operating conditions: discharge pressure 0.3 Torr, operating power $110 \mathrm{~W}$, pulse duration $5 \mathrm{~ms}$ with $25 \%$ duty cycle, and sampling distance $10 \mathrm{~mm}$.

Figure 4.3 shows mass spectra obtained from pure copper and $\mathrm{Nb}_{x} \mathrm{O}_{\mathrm{y}} / \mathrm{Cu}$ mixtures in the afterpeak regime (0.2 ms after power termination). For the pure copper pellet (Figure 4.3a), the ion intensities of copper increases dramatically compared to plateau regime. The copper dimers ( $\mathrm{m} / \mathrm{z} 126,128$ and 130) can also be seen as well as the copper ions at $\mathrm{m} / \mathrm{z} 63$ and $\mathrm{m} / \mathrm{z} 65$. In Figure 4.3b, 4.3c, and 4.3d, three NbxOy species exhibit the same niobium related ions $\left(\mathrm{Nb}^{+}, \mathrm{m} / \mathrm{z}\right.$ 93; $\left.\mathrm{NbO}^{+}, \mathrm{m} / \mathrm{z} 109 ; \mathrm{NbO}_{2}+, \mathrm{m} / \mathrm{z} 125\right)$, and their signals are also enhanced significantly. Characteristic ions of the different $\mathrm{Nb}_{x} \mathrm{O}_{\mathrm{y}}$ species 
are still not found in afterpeak regime. However, $\mathrm{NbO}, \mathrm{NbO}_{2}$, and $\mathrm{Nb}_{2} \mathrm{O}_{5}$ are found to yield different distributions of $\mathrm{Nb}_{\mathrm{x}} \mathrm{O}_{\mathrm{y}}$ related ions, so the ion intensity ratios between these ions can be used to differentiate the three $\mathrm{Nb}_{x} \mathrm{O}_{y}$ species. This will be discussed in the $\mathrm{Nb}_{x} \mathrm{O}_{y}$ speciation section.

The afterpeak regime was selected for niobium speciation because of the higher intensity of both atomic ions and cluster ions arising from niobium species. As mentioned earlier, Penning ionization is dominant during the afterpeak regime. In this regime, the sputtered niobium species population remains relatively stable, and ion intensities of analytes are related to the population of metastable argon atoms which is main ionization reagent in this regime..$^{25-26}$

To identify the optimal delay time to obtain the mass spectra for speciation, the signal of $\mathrm{Nb}^{+}, \mathrm{NbO}^{+}$, and $\mathrm{NbO}_{2}{ }^{+}$were monitored at different delay times using a pellet containing $25 \% \mathrm{NbO}$ and $75 \% \mathrm{Cu}$, as shown in Figure 4.4. Initially the signal intensities of analyte ion peaks increase with time and reach the maxima around 5.2 ms when the metastable argon population reaches the highest value. After $5.2 \mathrm{~ms}$, signals of both atomic ions and cluster ions decay continually and reach lower level at $5.4 \mathrm{~ms}$ because of the decline in the number of the metastable argon atoms. Therefore, 5.2 ms was found to afford the strongest signal of analyte ions. 


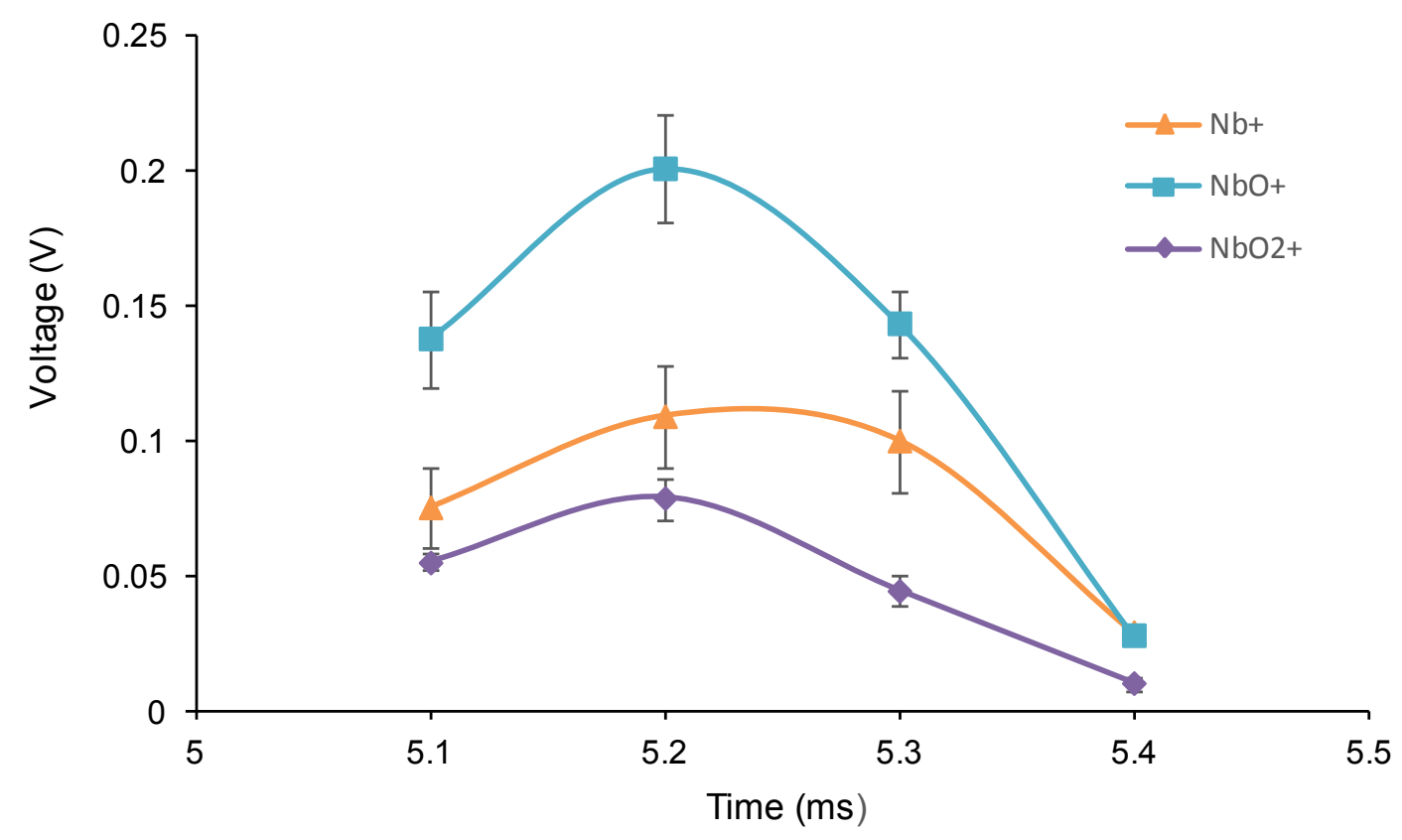

Figure 4.4 Effect of delay time on $\mathrm{Nb}^{+}, \mathrm{NbO}^{+}$and $\mathrm{NbO}^{2+}$ in afterpeak regime using $25 \% \mathrm{NbO}+75 \% \mathrm{Cu}$ pellet. Operating conditions: discharge pressure 0.3 Torr, operating power $110 \mathrm{~W}$, pulse duration $5 \mathrm{~ms}$ with $25 \%$ duty cycle, and sampling distance $10 \mathrm{~mm}$. Each data point is the average intensities of 3 measurements for each time.

\subsubsection{Evaluation of Sampling Distance}

The main analytical signal in mass spectrum comes from ions formed in the vicinity of ion exit orifice. Changing the sampling distance can therefore change the region of glow discharge plasma sampled and exhibit different ion signal profiles of analyte. ${ }^{14,} 27$ The sampling distance is adjusted by varying the distance between the cathode and ion exit orifice. To identify the optimal sampling distance for niobium speciation, the spatial distribution of atomic ions and cluster ions of $\mathrm{NbO}$ were examined by changing the 
sampling distance from $7 \mathrm{~mm}$ to $15 \mathrm{~mm}$. The plasma was operating at $110 \mathrm{~W}$ and 0.3 Torr with a delay time at $5.2 \mathrm{~ms}$.

Figure 4.5 shows the ion signal distribution of $\mathrm{Nb}^{+}, \mathrm{NbO}^{+}$, and $\mathrm{NbO}_{2}{ }^{+}$respectively with various sampling distance. Ion signals are very weak at $7 \mathrm{~mm}$. From 7 to $11 \mathrm{~mm}$, the intensity of atomic ion $\mathrm{Nb}^{+}$increases gradually and reaches the maximum at $11 \mathrm{~mm}$. Peak intensity then starts decreasing as distance further increases. Signal intensities of both cluster ions $\left(\mathrm{NbO}^{+}\right.$and $\left.\mathrm{NbO}_{2}{ }^{+}\right)$increase from $7 \mathrm{~mm}$ to $10 \mathrm{~mm}$, exhibit the maxima at $10 \mathrm{~mm}$, and then start to decrease at $11 \mathrm{~mm}$. The previous studies found that both the metastable argon atom population and Penning ionization maximized around 5-10 $\mathrm{mm}$ in the afterpeak regime. The similar result with previous studies further confirms that the cluster ions are generated mainly through Penning ionization. ${ }^{14,24}$ By contrast, the atomic ion $\mathrm{Nb}^{+}$signal exhibit the maximum value at the longer distance, which is consistent with the previous studies for metal oxide. ${ }^{6,16}$ This phenomenon indicates that the atomic ion is formed by both Penning ionization and dissociation of cluster ions. 


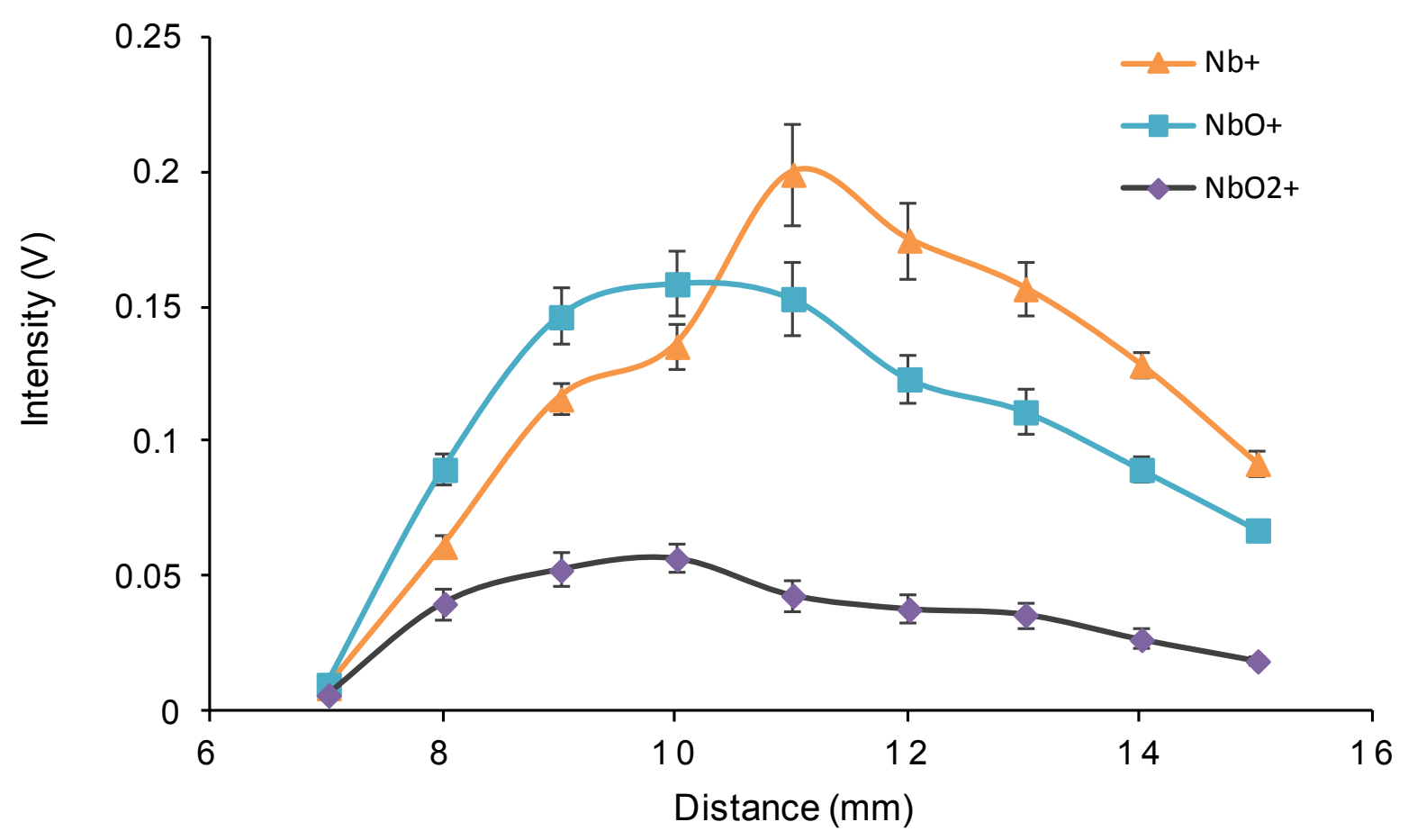

Figure 4.5 Effect of sampling distance on the ion signal profiles for representative $25 \% \mathrm{NbO}+75 \% \mathrm{Cu}$ pellet in afterpeak regime $(5.2 \mathrm{~ms})$. Operating conditions: discharge pressure 0.3 Torr, operating power $110 \mathrm{~W}$ and pulse duration $5 \mathrm{~ms}$ with $25 \%$ duty cycle. Each data point is the average intensities of 3 measurements for each distance.

\subsubsection{Evaluation of Operating Power}

The previous work in our lab found that the operating power can affect ion signal intensities significantly. $6,14,16$ The effect of power on ion intensities was examined by changing the operating power from $90 \mathrm{~W}$ to $130 \mathrm{~W}$. The sampling distance was fixed at $10 \mathrm{~mm}$, and the discharge gas pressure remained at 0.3 Torr. The representative sample used for this test was $25 \% \mathrm{NbO}+75 \%$ Cu pellet. At power below $90 \mathrm{~W}$, the signal was very weak. The Figure 4.6 shows the intensities of $\mathrm{Nb}^{+}, \mathrm{NbO}^{+}$, and $\mathrm{NbO}_{2}{ }^{+}$versus 
operating power in afterpeak regime. The intensity of $\mathrm{Nb}^{+}$keeps on increasing as higher power is applied because the increased operating power not only increases sputter rate, but also increases the population of metastable argon atoms used for Penning ionization. $\mathrm{NbO}^{+}$ion intensity increases from $90 \mathrm{~W}$ to $120 \mathrm{~W}$ and reaches the maximum at $120 \mathrm{~W}$. The $\mathrm{NbO}_{2}{ }^{+}$exhibits similar trend and reaches the maximum at $110 \mathrm{~W}$. These results indicate that increasing the power may increase the possibility of cluster ion dissociation because of more energetic species in the negative glow. In addition, the ion signals of niobium species tend to be unstable when the operating power is higher than $110 \mathrm{~W}$. 


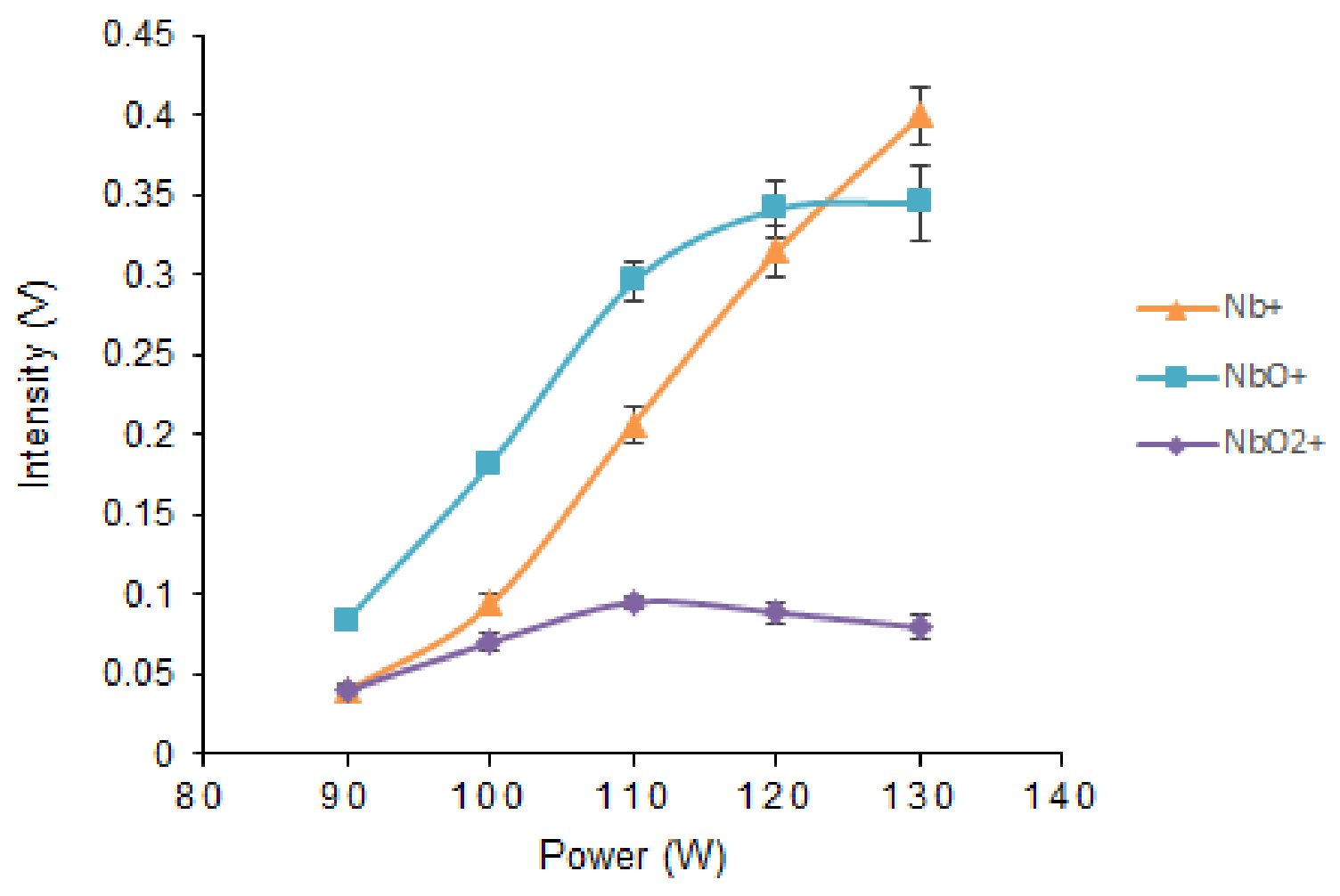

Figure 4.6 Effect of operating power on $\mathrm{Nb}^{+}, \mathrm{NbO}^{+}$and $\mathrm{NbO}^{2+}$ in afterpeak regime (5.2 ms) using $25 \% \mathrm{NbO}+75 \% \mathrm{Cu}$ pellet. Operating conditions: discharge pressure $0.3 \mathrm{Torr}$, pulse duration $5 \mathrm{~ms}$ with $25 \%$ duty cycle, and sampling distance $10 \mathrm{~mm}$. Each data point is the average intensities of 3 measurements for each operating power.

\subsubsection{Speciation of Niobium Oxides $\left(\mathrm{Nb}_{\mathrm{x}} \mathrm{O}_{\mathrm{y}}\right)$}

As discussed above, no characteristic ions were found for any niobium oxide species. $\mathrm{NbO}, \mathrm{NbO}_{2}$, and $\mathrm{Nb}_{2} \mathrm{O}_{5}$ all exhibit similar mass spectra in afterpeak regime with $\mathrm{Nb}^{+}, \mathrm{NbO}^{+}$, and $\mathrm{NbO}_{2}{ }^{+}\left(\mathrm{m} / \mathrm{z} 93,109\right.$ and 125). In the past, it was found that $\mathrm{NbO}_{2}{ }^{+}$is the most stable niobium cluster ions $\mathrm{Nb}_{x} \mathrm{O}_{y}{ }^{+}(x \geq 2)$ in both theoretical and experimental studies.$^{28-29}$ The observation of $\mathrm{NbO}_{2}{ }^{+}$in present work is consistent with the previous studies for stability of niobium cluster ions. Nevertheless, larger niobium cluster ions, 
which were previously observed in the experimental study, are not shown in the mass spectra (Figure 4.3) of the present work. However, the method to form cluster ions is different from that in our study, and they used laser ablation of pure niobium metal to react with oxygen to form niobium cluster ions. ${ }^{29}$ Compared with glow discharge, this method provides a cooler collision environment, which favored survival of larger cluster ions. It may because of lower sputtering rate for larger neutral niobium oxide clusters and the dissociation of larger sputtered neutral clusters and larger cluster ions. In Dong's study, the neutral niobium oxide clusters $\left(\mathrm{Nb}_{\mathrm{x}} \mathrm{O}_{\mathrm{y}}\right)$, which were formed in laser induced plasma, generated $\mathrm{Nb}^{+}$and small cluster ions $\mathrm{NbO}^{+}$, and $\mathrm{NbO}_{2}{ }^{+}$using $193 \mathrm{~nm}$ multi-photon ionization, which is similar to our observation. ${ }^{30}$ Chen et al. compacted $\mathrm{Nb}_{2} \mathrm{O}_{5}$ powder into a pellet following by ablation of $\mathrm{Nb}_{2} \mathrm{O}_{5}$ pellet in pure argon gas using $532 \mathrm{~nm}$ laser. They also only observed small cluster ions using this method which is more similar with our method. ${ }^{31}$

As mentioned in previous disccusion, Penning ionization is the main ionization mechanism in the glow discharge plasma during the afterpeak regime. The main mechanism for formation of $\mathrm{NbO}^{+}$and $\mathrm{NbO}_{2}{ }^{+}$would be expected as Penning ionization of neutral $\mathrm{NbO}$ and $\mathrm{NbO}_{2}$ :

$$
\begin{gathered}
\mathrm{NbO}+\mathrm{Ar}^{*} \rightarrow \mathrm{NbO}^{+}+\mathrm{Ar}+\mathrm{e}^{-} \\
\mathrm{NbO}_{2}+\mathrm{Ar}^{*} \rightarrow \mathrm{NbO}_{2}^{+}+\mathrm{Ar}+\mathrm{e}^{-}
\end{gathered}
$$

The source of neutral $\mathrm{NbO}$ and $\mathrm{NbO}_{2}$ is related to the species released from the cathode. The cathode sputtering process can induce releasing of neutral atoms and molecules, cluster of cathode material, and electrons. Therefore, the neutral $\mathrm{NbO}$ and 
$\mathrm{NbO}_{2}$ can be generated from direct cathodic sputtering, recombination of sputtered $\mathrm{Nb}$ and $\mathrm{O}$ or $\mathrm{NbO}$ and $\mathrm{O}$, and dissociation of larger neutral $\mathrm{Nb}_{x} \mathrm{O}_{y}$. Besides penning ionization, it is possible to form $\mathrm{NbO}^{+}$and $\mathrm{NbO}_{2}{ }^{+}$by some aggregation mechanism such as following reactions:

$$
\begin{aligned}
& \mathrm{Nb}^{+}+\mathrm{O} \rightarrow \mathrm{NbO}+ \\
& \mathrm{NbO}^{+}+\mathrm{O} \rightarrow \mathrm{NbO}_{2}^{+}
\end{aligned}
$$

The proportion of these mechanisms can be changed by experimental conditions. However, the intensity of niobium oxide cluster ions should relate to the abundance of oxygen in the niobium oxide. The previous studies also found that the stoichiometry and valence of metal oxide show a strong correlation with relative abundance distribution of clusters ions and atomic ions. ${ }^{9-11,16}$ It is expected that the metal oxide species with higher oxygen content should have relative higher abundance ratio of metal oxide cluster ions over atomic ions. Therefore, the ratio of $\mathrm{NbO}^{+}(x=1,2)$ and $\mathrm{Nb}^{+}$can be used to differentiate three niobium oxides. The results are shown in Figure 4.7, which is consistent with the proposed phenomenon. $\mathrm{NbO}$ with lowest oxygen abundance exhibits the lowest ratio (2.531); the ratio of $\mathrm{NbO}_{2}$ is in the middle (3.195), and $\mathrm{Nb}_{2} \mathrm{O}_{5}$ with highest oxygen content shows the highest ratio (4.031). 


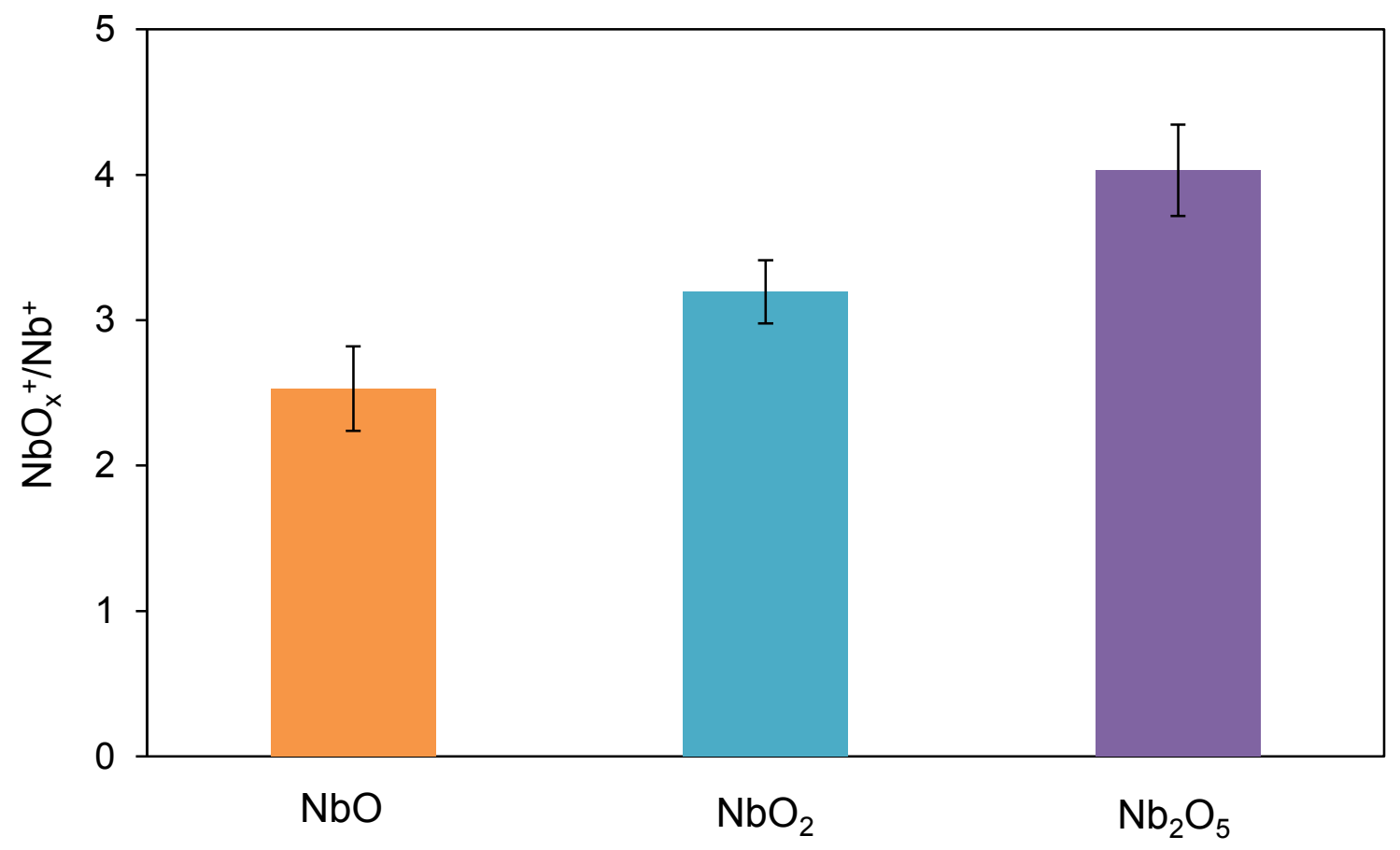

Figure 4.7 $\mathrm{NbO}_{x}^{+}(x=1,2)$ and $\mathrm{Nb}^{+}$peak intensity ratios for $\mathrm{NbO}, \mathrm{NbO}_{2}$, and $\mathrm{Nb}_{2} \mathrm{O}_{5}$ during the afterpeak (5.2 ms). Operating conditions: discharge pressure 0.3 Torr, operating power $110 \mathrm{~W}$, pulse duration $5 \mathrm{~ms}$ with $25 \%$ duty cycle, and sampling distance $10 \mathrm{~mm}$. Each data point is the average of 6 measurements for each species 


\subsection{Conclusions}

With careful control of operating parameters of pulsed glow discharge time-of-flight mass spectrometry, the resulting ion signal profiles enable the niobium oxides speciation. It is found that the stoichiometry of niobium oxides show a strong correlation with relative abundance distribution of niobium oxide clusters ions and niobium atomic ions. The niobium oxide species with higher oxygen content exhibit higher abundance ratio of metal oxide cluster ions versus atomic ions. The different ion abundance ratios permit direct speciation of $\mathrm{NbO}, \mathrm{NbO}_{2}$, and $\mathrm{Nb}_{2} \mathrm{O}_{5}$ in solid state samples. 


\subsection{References}

1. Froideval, A.; Degueldre, C.; Segre, C. U.; Pouchon, M. A.; Grolimund, D., Niobium Speciation at the Metal/Oxide Interface of Corroded Niobium-Doped Zircaloys: A X-ray Absorption Near-Edge Structure Study, Corrosion Science, 2008, 50, 13131320.

2. Fliegel, D.; Waddell, R.; Majidi, V.; Günther, D.; Lewis, C. L., Quantification of Aromatic and Halogenated Hydrocarbons and Alcohol Mixtures at the Elemental, Structural, and Parent molecular Ion Level, Anal. Chem., 2005, 77, 1847-1852.

3. Wu, A. T., Investigation of Oxide Layer Structure On Niobium Surface Using a Secondary Ion Mass Spectrometry, Physica C: Superconductivity and Its Applications, 2006, 441, 79-82.

4. Ure, A. M.; Davidson, C. M.; Chemical Speciation in the Environment, Blackie Academic \& Professional, London, 1995.

5. Robertson-Honecker, J. N.; Zhang, N.; Pavkovich, Alexandria; King, F. L., Direct Chromium Speciation in Solid State Materials-a GDMS Approach, J. Anal. At. Spectrom., 2008, 23, 1508-1517.

6. Zhang, N.; King, F. L., Direct Manganese (Mn) Speciation in Solid State Materials by Pulsed Glow Discharge Time-of-Flight Mass Spectrometry, J. Anal. At. Spectrom., 2009, 24, 1489-1497. 
7. Aubriet, F.; Poleunis, C.; Bertrand, P., Capabilities of Static TOF-SIMS in the Differentiation of First-Row Transition Metal Oxides, J. Mass Spectrom., 2001, 36, $641-651$.

8. Aubriet, F.; Maunit, B.; Courrier, B8; Muller, J. F., Studies of the Chromium Oxygenated Cluster lons Produced During the Laser Ablation of Chromium Oxides by Laser Ablation/lonization Fourier Transform Ion Cyclotron Resonance Mass Spectrometry, Rapid Commun. Mass Spectrom., 1997, 11, 1596-1601.

9. Maunit, B.; Hachimi, A.; Manuelli, P.; Calba, P. J.; Muller, J. F., Formation of Iron Oxides Clusters induced by Resonant Laser Ablation/Ionization, International Journal of Mass Spectrometry and Ion Processes, 1996, 156, 173-187.

10. Michiels, E.; Gijbels, R., Fingerprint Spectra in Laser Microprobe Mass Analysis of Titanium Oxides of Different Stoichiometry, Spectrochimica Acta, 1983, 38B, $1347-1354$.

11. Michiels, E.; Gijbels, R., Cluster Ion Distributions and Correlation with Fragment Valence in Laser-Induced Mass Spectra of Oxides, Analytical Chemistry, 1984, 56, $1115-21$.

12. King, F. L.; Teng, J. ; Steiner, R. E., Glow Discharge Mass Spectrometry: Trace Element Determinations in Solid Samples, Journal of Mass Spectrometry, 1995, 30,1061-1075.

13. Pan, C.; King, F. L., Time-Resolved Studies of lonized Sputtered Atoms in Pulsed Radio Frequency Powered Glow Discharge Mass Spectrometry, Anal. Chem., 1993, 65, 3187-3193. 
14. Li, L.; Millay, J. T.; Turner, J. P.; King, F. L., Millisecond Pulsed Radio Frequency Glow Discharge Time of Flight Mass Spectrometry: Temporal and Spatial Variations in Molecular Energetics, J. Am. Soc. Mass Spectrom., 2004, 15, 87-102.

15. Lewis, C. L.; Moser, M. A.; Dale Jr, D. E.; Hang, W.; Hassell, C.; King, F. L.; Majidi, V., Time-Gated Pulsed Glow Discharge: Real-Time Chemical Speciation at the Elemental, Structural, and Molecular Level for Gas Chromatography Time-of-Flight Mass Spectrometry, Anal. Chem., 2003, 75, 1983-1996.

16. Gu, G.; DeJesus, M.; King, F. L., Direct FexOy Speciation in Solid State Materials by Pulsed Millisecond Radio Frequency Glow Discharge Time-of-Flight Mass Spectrometry, J. Anal. At. Spectrom., 2011, 26, 816-821.

17. Batchelor, A. D.; Leonard, D. N.; Russell, P. E.; Stevie, F. A.; Griffis, D. P.; Myneni, G. R. TEM and SIMS Analysis of (100), (110), and (111) Single Crystal Niobium, AIP Conference Proceedings, 2007, 927(Single Crystal - Large Grain Niobium Technology), 72-83.

18. Li, S.; Hu, B.; Jiang, Z., Direct Determination of Trace Impurities in Niobium Pentaoxide Solid Powder with Slurry Sampling Fluorination Assisted Electrothermal Vaporization Inductively Coupled Plasma Mass Spectrometry, J. Anal. At. Spectrom., 2004, 19,387-391.

19. Dong, F.; Heinbuch, S.; Bernstein, E. R.; Rocca, J. J., Mass Spectroscopy of Neutral Metal Oxide Clusters Using a Desk-Top Soft X-Ray Laser, Springer Proceedings in Physics, 2007, 115(X-Ray Lasers 2006), 463-469. 
20. Chen, M.; Wang, X.; Qin, Q, Characterization of the Products Generated from Laser Ablation of $\mathrm{Nb}_{2} \mathrm{O}_{5}$ Using Matrix Isolation FTIR Spectroscopy, Applied Surface Science 2000, 156, 16-20.

21. Lim, J. H.; Choi, J., Formation of Niobium Oxide Nanowires by Thermal Oxidation, Journal of Industrial and Engineering Chemistry, 2009, 15, 860-864.

22. Steiner, R. E.; Lewis, C. L.; King, F. L., Time-of-Flight Mass Spectrometry with a Pulsed Glow Discharge Ionization Source, Anal. Chem., 1997, 69, 1715-1721.

23. Klinger, J. A.; Savickas, P. J.; Harrison W. W., The Pulsed Glow Discharge as an Elemental Ion Source, J. Am. Soc. Mass Spectrom., 1990, 1, 138-143.

24. Jackson, G. P.; Lewis, C. L.; Doorn, S. K.; Majidi, V.; King, F. L., Spectral, Spatial, and Temporal Characteristics of a Milliosecond Glow Discharge: Metastable Argon Atom Production., Spectrochim. Acta, 2001, 56B, 2449-2464.

25. Lewis, C. L.; Jackson, G. P.; Doorn, S. K.; Majidi, V.; King, F. L., Spectral, Spatial and Temporal Characterization of a Millisecond Pulsed Glow Discharge: Copper Analyte Emission and Ionization, Spectrochimica Acta, 2001, 56B, 487-501.

26. Pan, C.; King, F. L., Ion Formation Processes in the Afterpeak Time Regime of Pulsed Glow Discharge Plasmas, J. Am. Soc. Mass Spectrom., 1993, 4, 727-732.

27. DeJesus, M. R.; Gu, G.; King, F. L.; Barnes, J. H.; Lewis, C. L., Ion formation in millisecond pulsed glow discharge plasmas, , J. Anal. At. Spectrom., 2011, 26, 2206-2215.

28. Sambrano, J. R.; Andres, J.; Beltran, A.; Sensato, F.; Longo, E., Theoretical Study of the Structure and Stability of $\mathrm{Nb}_{x} \mathrm{O}_{y}$ and $\mathrm{Nb}_{x} \mathrm{O}_{y}{ }^{+}(x=1-3 ; y=2-5,7,8)$ clusters, Chemical Physics Letters, 1998, 287, 620-626. 
29. Deng, H. T.; Kerns, K. P.; Castleman, A. W., Jr., Formation, Structures, and Reactivities of Niobium Oxide Cluster lons, Journal of Physical Chemistry, 1996, $100,13386-13392$.

30. Dong, F.; Heinbuch, S.; He, S. G.; Xie, Y.; Rocca, J. J.; Bernstein, E. R., Formation and Distribution of Neutral Vanadium, Niobium, and Tantalum Oxide Clusters: Single Photon Ionization at $26.5 \mathrm{eV}$, Journal of Chemical Physics, 2006, 125, $164318 / 1-164318 / 8$.

31. Chen, M.; Wang, X.; Qin, Q., Characterization of the Products Generated from Laser Ablation of Nb2O5 Using Matrix Isolation FTIR Spectroscopy, Applied Surface Science, 2000, 156, 16-20. 